\title{
THREE-DIMENSIONAL TOPOLOGICAL FIELD THEORY AND SYMPLECTIC ALGEBRAIC GEOMETRY I
}

\author{
ANTON KAPUSTIN, LEV ROZANSKY, AND NATALIA SAULINA
}

\begin{abstract}
We study boundary conditions and defects in a threedimensional topological sigma-model with a complex symplectic target space $X$ (the Rozansky-Witten model). We show that boundary conditions correspond to complex Lagrangian submanifolds in $X$ equipped with complex fibrations. The set of boundary conditions has the structure of a 2-category; morphisms in this 2category are interpreted physically as one-dimensional defect lines separating parts of the boundary with different boundary conditions. This 2-category is a categorification of the $\mathbb{Z}_{2}$-graded derived category of $X$; it is also related to categories of matrix factorizations and a categorification of deformation quantization (quantization of symmetric monoidal categories). In the appendix we describe a deformation of the B-model and the associated category of branes by forms of arbitrary even degree.
\end{abstract}




\section{Contents}

1. Introduction

2. Topological boundary conditions in the RW model

2.1. Review of the RW model

2.2. Reduction of the RW model on a circle 7

2.3. Complex lagrangian submanifolds 9

2.4. Deformations of boundary conditions 11

2.5. Calabi-Yau fibrations 20

2.6. Reduction on a circle in the presence of boundaries 33

2.7. Reduction on an interval 34

3. Topological defects $\quad 45$

3.1. Surface operators $\quad 45$

3.2. Line operators 48

3.3. Point operators 58

4. Relationship with categorified algebraic geometry 60

Appendix A. The B-model and its category of branes 63

A.1. BRST transformations, action, and observables 63

A.2. Topological branes in the B-model 64

Appendix B. The curved B-model and its category of branes 67

B.1. BRST transformations, action, and observables $\quad 67$

B.2. Topological branes in the curved B-model 69

Appendix C. Perturbative computation of the fusion of Wilson lines on the boundary $\quad 72$

References

\section{INTRODUCTION}

Topological field theory (TFT) provides a bridge between quantum field theory with its nonrigorous path-integrals and rigorous mathematics. Two-dimensional topological field theories have been studied in great detail, mostly in connection with mirror symmetry. Threedimensional topological field theories are much less understood, with the exception of the Chern-Simons gauge theory [41]. But ChernSimons theory is a rather degenerate example of a $3 \mathrm{~d}$ TFT. It has no nontrivial local observables or boundary conditions; the only topological observables are Wilson loops which are localized on closed 1dimensional submanifolds. Our experience with 2d TFT teaches us that a theory is much more interesting and nontrivial when one can define it on a manifold with boundaries. 
In this paper we study a 3d TFT of a geometric nature which admits nontrivial boundary conditions. This theory is a topological sigmamodel which was introduced by E. Witten and the second author in [32]. We will call it the RW model. The target manifold for such a model must be a hyper-Kähler manifold $X$, or more generally a holomorphic symplectic manifold. This theory is closely related to the B-model with target $X$ and reduces to it upon compactification on a circle. We will see that it admits a variety of boundary conditions which are analogous to topological branes in the B-model.

There are several reasons to study this model. Firstly, it is rich enough to elucidate the general structure of $3 \mathrm{~d}$ TFT. The original definition of a TFT in arbitrary dimension given by M. Atiyah [1] did not allow for boundaries; neither did it allow for defects of higher codimension (such as Wilson loops in Chern-Simons theory). It was realized in mid 90's that such a generalization is rather intricate and is most conveniently formulated in the language of category theory. In two dimensions examples of the resulting structure are provided by $\mathrm{A}$ and B-models associated to Calabi-Yau manifolds [42, 13], and more abstractly by Calabi-Yau $A_{\infty}$ categories $[26,10]$. The structure of $3 \mathrm{~d}$ TFT is even more complex and requires higher category theory (see e.g. $[14,2,11])$, but concrete examples have been sparse. The path-integral for the RW model can be regarded as a heuristic tool for constructing such an example, or rather, a class of examples.

Another mathematical reason to study the RW model with boundaries is its relationship with the problem of "deformation quantization" of the derived category of coherent sheaves on a complex manifold, regarded as a symmetric monoidal category. This relationship will be explained below. We also find a close connection between RW theory and categorified algebraic geometry [36, 4]. Namely, in the case when the target space of the RW model has the form $T_{Y}^{*}$, where $Y$ is a complex manifold, the 2-category of boundary conditions is very similar to the 2-category of derived categorical sheaves on $Y$ as defined in [36]. This will discussed in detail in the follow-up paper [23].

Finally, a physical motivation to study the RW model is its connection with the $3 \mathrm{~d}$ mirror symmetry of K. Intriligator and N. Seiberg [17]. This is a conjectural duality between $3 \mathrm{~d}$ gauge theories with $N=4$ supersymmetry. An $N=4 d=3$ theory can be twisted into a $3 \mathrm{~d}$ topological field theory, and in some special cases the twisted theory turns out to be equivalent to the RW model (with a noncompact target space $X$ ). Understanding the RW model thus represents a step towards understanding $3 \mathrm{~d}$ mirror symmetry. 
Here is a brief summary of the results of the paper. We show that the simplest topological boundary conditions in the RW model with target $X$ correspond to complex Lagrangian submanifolds of $X$. More generally, we show how to associate a boundary condition to a fibration over a complex Lagrangian submanifold $Y$ whose fiber is a Calabi-Yau manifold $Z$. One should think of this fibration as a B-model with target $Z$ fibered over $Y$. This already points to a connection with derived algebraic geometry, which we discuss in section 4 . Even more generally, one might consider fibering over $Y$ a deformation of the B-model by forms of even degrees. For example, if the form is a holomorphic function on $Z$, one gets a fibered Landau-Ginzburg model. We argue that such a generalization is necessary if we want the set of boundary conditions to be closed with respect to some natural operations.

The set of boundary conditions for the RW model with target $X$ should be thought of as the set of objects of a 2-category. The set of morphisms from one boundary condition to another is interpreted physically as the set of topological defects of real dimension one separating one boundary condition from the other. We call such defects boundary line operators; they are similar to Wilson line operators in Chern-Simons theory and in the twisted $N=4 d=4$ gauge theory considered in [24]. Boundary line operators corresponding to a pair of boundary conditions form a category; we show that it is closely related to a $\mathbb{Z}_{2}$-graded version of the derived category of coherent sheaves. In certain cases, including the case when $X=T_{Y}^{*}$ for some complex manifold $Y$, one can promote $\mathbb{Z}_{2}$-grading to $\mathbb{Z}$-grading.

If the two boundary conditions coincide, the corresponding category of boundary line operators has a monoidal structure, i.e. an associative tensor product. Physically, the composition of line operators arises from fusing them together. This composition is noncommutative in general. It is a deformation of the usual tensor product of holomorphic vector bundles on $Y$.

Let us say a few words about the organization of the paper. In section 2 we study boundary conditions in the RW model. In section 3 we study surface operators, line operators, and point operators. In section 4 we discuss the algebraic interpretation of the 2-category of boundary conditions in the RW model. We argue that it is best expressed in the language of categorified algebraic geometry as discussed recently in $[36,4]$. In the appendix we review the B-model and introduce a generalization of the B-model which we call the curved B-model. The category of branes for the curved B-model plays a central role in this paper and is a deformation the $\mathbb{Z}_{2}$-graded derived category of coherent sheaves. 
In the second part of this paper [23] we will reformulate our results in the language of the Atiyah-Segal axiomatic approach to Topological Field Theory. We will also discuss the relationship between the RW model and categorified algebraic geometry.

A. K. would like to thank Alexei Bondal, Dennis Gaitsgory, David Ben-Zvi, David Nadler, Dan Freed, Andrei Mikhailov, and Dima Orlov for discussions. L. R. would like to thank Dima Arinkin, David BenZvi and David Nadler for discussions. N. S. would like to thank Andrei Mikhailov for discussions. Part of this work was done during the BIRS workshop "Matrix Factorizations in Physics and Mathematics", May 2008. A. K. and L. R. are grateful to the organizers for the invitation and to the Banff International Research Station for hospitality. The work of A. K. and N. S. was supported in part by the DOE grant DEFG03-92-ER40701. The work of L. R. was supported by NSF grant DMS-0808974.

\section{TOPOLOGICAL BOUNDARY CONDITIONS IN THE RW MODEL}

2.1. Review of the RW model. Let $M$ be a Riemannian 3-manifold with local coordinates $x^{\mu}, \mu=1,2,3$, and $X$ be a hyper-Kähler manifold $X$ of complex dimension $\operatorname{dim}_{\mathbb{C}} X=2 n$. The fields of the theory are

$$
\text { bosonic: } \quad \phi^{I}, \phi^{\bar{I}}, \quad \text { fermionic: } \quad \eta^{\bar{I}}, \chi_{\mu}^{I} \text {. }
$$

where $I, \bar{I}=1, \ldots, 2 n, \quad \mu=1,2,3$. Bosonic fields $\phi^{I}(x), \phi^{\bar{I}}(x)$ describe a map $\phi$ from $M$ to $X$ (in complex coordinates). The fermionic fields $\chi_{\mu}^{I}$ are components of a 1 -form $\chi^{I}$ on $M$ with values in $\phi^{*}\left(T_{X}\right)$, where $T_{X}$ is the holomorphic tangent bundle of $X$. The fermionic field $\eta^{\bar{I}}$ is a 0 -form on $M$ with values in the complex-conjugate bundle $\phi^{*}\left(\bar{T}_{X}\right)$. BRST transformations of the fields are

$$
\delta_{Q} \phi^{I}=0, \quad \delta_{Q} \phi^{\bar{I}}=\eta^{\bar{I}}, \quad \delta_{Q} \eta^{\bar{I}}=0, \quad \delta_{Q} \chi^{I}=d \phi^{I} .
$$

This transformation is nilpotent, $\delta_{Q}^{2}=0$, and is a derivation of the algebra generated by fields and their derivatives. The cohomology of $\delta_{Q}$ is called the algebra of topological observables.

The BRST invariant action consists of two parts:

$$
S=\int_{M} \mathcal{L}=\int_{M} \mathcal{L}_{1}+\int_{M} \mathcal{L}_{2}
$$


where

$$
\begin{aligned}
& \mathcal{L}_{1}=\delta_{Q}\left(g_{I \bar{J}} \chi^{I} \wedge * d \phi^{\bar{J}}\right)=g_{I \bar{J}} d \phi^{I} \wedge * d \phi^{\bar{J}}-g_{I \bar{J}} \chi^{I} \wedge * \nabla \eta^{\bar{J}}, \\
& \mathcal{L}_{2}=\frac{1}{2} \Omega_{I J}\left(\chi^{I} \wedge \nabla \chi^{J}+\frac{1}{3} \mathcal{R}_{K L \bar{M}}^{J} \chi^{I} \wedge \chi^{K} \wedge \chi^{L} \wedge \eta^{\bar{M}}\right) .
\end{aligned}
$$

Here $\Omega_{I J}$ is the holomorphic symplectic form on $X$, star denotes the Hodge star operator on forms on $M$ with respect to a Riemannian metric $h_{\mu \nu}$, and the covariant derivatives are defined using the pullback of the Levi-Civita connection:

$$
\begin{aligned}
& \nabla \eta^{\bar{J}}=d \eta^{\bar{J}}+\Gamma_{\bar{I} \bar{K}}^{\bar{J}} d \phi^{\bar{I}} \wedge \eta^{\bar{K}}, \\
& \nabla \chi^{J}=d \chi^{J}+\Gamma_{I K}^{J} d \phi^{I} \wedge \chi^{K} .
\end{aligned}
$$

(From now on, we will omit the sign $\wedge$ when writing the exterior product of forms on $M$.) Finally, $\mathcal{R}_{K L \bar{M}}^{J}$ denotes the curvature tensor of the Levi-Civita connection on $X$ :

$$
\mathcal{R}_{K L \bar{M}}^{J}=\frac{\partial \Gamma_{K L}^{J}}{\partial \phi^{\bar{M}}}, \quad \Gamma_{J K}^{I}=\left(\partial_{J} g_{K \bar{M}}\right) g^{I \bar{M}} .
$$

Note that only the BRST exact piece $\mathcal{L}_{1}^{b u l k}$ depends on the choice of the metric $h_{\mu \nu}$ on $M$. Therefore the partition function and correlators of BRST-invariant observables are independent of the metric. In other words, the theory is a topological field theory.

Correlators of BRST-invariant observables are called topological correlators. As usual, any topological correlator involving a BRST-exact observable vanishes. Thus one may regard topological correlators as multilinear functions on the BRST cohomology, i.e. on the algebra of topological observables. The algebra of local topological observables for the RW model is isomorphic to

$$
\oplus_{p} H^{p}\left(\mathcal{O}_{X}\right) .
$$

Indeed, given a $(0, p)$-form $\omega_{\bar{I}_{1} \ldots \bar{I}_{p}}$ we let

$$
\mathcal{O}_{\omega}=\frac{1}{p !} \omega_{\bar{I}_{1} \ldots \bar{I}_{p}} \eta^{\bar{I}_{1}} \ldots \eta^{\bar{I}_{p}}
$$

Then the BRST-operator acts by

$$
\delta_{Q}\left(\mathcal{O}_{\omega}\right)=\mathcal{O}_{\bar{\partial} \omega}
$$

and therefore the BRST cohomology is isomorphic to the Dolbeault cohomology. The algebra structure is given by the ordinary exterior product of forms. ${ }^{1}$

\footnotetext{
${ }^{1}$ This analysis is classical, but we will show below that no quantum corrections are possible.
} 
The term $\mathcal{L}_{2}$ in the Lagrangian depends only on the holomorphic symplectic form $\Omega$ on $X$. Thus topological correlators do not depend on the Kähler form of $X$ and are holomorphic functions of $\Omega$. In fact, it is not necessary to require the $(1,1)$ form $g_{I \bar{J}}$ to be closed, and the theory makes sense when $X$ is a not-necessarily-Kähler complex symplectic manifold. The action is a bit more complicated since the natural connection in the non-Kähler case is not the Levi-Civita connection. To simplify formulas, in the rest of the paper we will assume that $X$ is hyper-Kähler. However, all the results hold for arbitrary complex symplectic manifolds.

As a consequence of BRST symmetry, the path-integral computing topological correlators localizes on BRST-invariant field configurations. These can be determined by requiring the BRST variations of fermionic fields to vanish. From $\delta_{Q} \chi^{I}=d \phi^{I}$ it follows that the path-integral localizes on constant maps to $M$. The contribution of a particular map is given by the ratio of certain determinants (the Ray-Singer torsion) times the sum of Feynman diagrams; the Feynman rules are explained in detail in [32]. The correlator is obtained by integrating over the moduli space of constant maps to $X$, i.e. over $X$.

In addition to BRST symmetry, the theory also has a $\mathbb{Z}_{2}$ symmetry which multiplies all fermionic fields by -1 . If the Lagrangian contained only the $\mathcal{L}_{1}$ term, this $\mathbb{Z}_{2}$ could be extended to a $U(1)$ symmetry, so that the field $\chi$ has $U(1)$ charge -1 and $\eta$ has charge +1 . This symmetry is broken by $\mathcal{L}_{2}$ down to $\mathbb{Z}_{2}$. One can formally restore it by assigning the holomorphic symplectic form $\Omega$ charge +2 . We will call this symmetry the ghost number symmetry.

The ghost number symmetry has interesting consequences for the dependence of the partition function on the symplectic form $\Omega$. If the measure in the path-integral were invariant under ghost number symmetry, one could conclude that the partition function is invariant under a rescaling of $\Omega$ and therefore, by holomorphy, independent of $\Omega$. As a matter of fact, because of fermionic zero modes the measure has charge $2 n\left(b_{1}(M)+1\right)$ [32], so under the rescaling

$$
\Omega \mapsto \lambda^{2} \Omega
$$

the partition function scales as

$$
Z(M) \mapsto \lambda^{2 n\left(b_{1}(M)+1\right)} Z(M) .
$$

As explained in [32], this scaling implies that for a particular $M$ only a finite number of Feynman diagrams may contribute to the partition function. 
From the physical viewpoint, it is convenient to redefine

$$
\Omega \mapsto \hbar^{-1} \Omega, \quad g_{I \bar{J}} \mapsto \hbar^{-1} g_{I \bar{J}},
$$

so that the action (3) is proportional to $\hbar^{-1}$ and one can interpret $\hbar$ as the Planck constant. The above discussion of ghost number symmetry can be restated by saying that topological correlators of observables with a fixed ghost number receive contributions only from a particular order in the $\hbar$-expansion. In what follows, we will often deal with observables which do not have a definite ghost number. Correlators of such observables are formal power series in $\hbar$. However, for target $X$ of a fixed dimension no problems with convergence arise, since higher powers of $\hbar$ are accompanied by higher powers of fermionic fields, and there is only a finite number of the latter.

2.2. Reduction of the RW model on a circle. If $M=S^{1} \times \Sigma$, where $\Sigma$ is a two-dimensional oriented manifold, the RW model reduces to the B-model on $\Sigma$ with target $X$ [33]. Indeed, since the model is topological, we may assume that the size of the circle is very small, Fourier-expand all the fields on the circle and retain only the constant modes. The field $\eta^{\bar{I}}$ becomes a 0 -form on $\Sigma$. If we denote by $x^{3}$ the coordinate on $S^{1}$, the 1 -form field $\chi^{I}$ can be expanded as

$$
\chi^{I}=\chi_{\Sigma}^{I}+\chi_{3}^{I} d x^{3},
$$

where $\chi_{\Sigma}^{I}$ is a pull-back of a 1 -form $\rho^{I}$ on $\Sigma$. As for the field $\chi_{3}^{I}$, it becomes a 0 -form on $\Sigma$. We can define

$$
\theta_{I}=\Omega_{I J} \chi_{3}^{J} \text {. }
$$

Note that since $\Omega_{I J}$ has ghost number 2 , the fields $\theta_{I}$ and $\eta^{\bar{I}}$ have ghost number 1 , while $\rho^{I}$ has ghost number -1 . Thus the ghost number symmetry of the RW model becomes the usual ghost number symmetry of the B-model.

The BRST transformation restricted to constant Fourier modes read

$$
\begin{array}{rlrl}
\delta \phi^{I} & =0, & \delta \phi^{\bar{I}}=\eta^{\bar{I}}, \\
\delta \eta^{\bar{I}}=0, & \delta \theta_{I}=0, \\
\delta \rho^{I}=d \phi^{I} . &
\end{array}
$$

These are the BRST transformations of the B-model with target $X$. It is easy to check that the action of the RW model also reduces to the action of the B-model. In what follows we will often use the known properties of the B-model as a guide to understanding the RW model.

The above analysis of the reduction was classical. In the quantum theory, instead of setting nonconstant Fourier modes to zero, one has 
to integrate over them in the path-integral. This could induce corrections to the action of the B-model. However, using the ghost number symmetry, it is easy to show that no such corrections are possible, and the above classical result is exact. Indeed, infinitesimal deformations of the B-model with target $X$ are parameterized by even elements of the $\mathbb{Z}_{2}$-graded vector space

$$
\oplus_{p, q} H^{p}\left(\Lambda^{q} T_{X}\right)
$$

Since the RW model on $S^{1} \times \Sigma$ has ghost number symmetry, the action of the reduced model also should preserve this symmetry. This implies that possible deformations of the B-model must have $p+q=2$. On the other hand, the class in question must be constructed from the curvature of $X$, which is an element of $H^{1}\left(\Omega^{1} \otimes\right.$ End $\left.T_{X}\right)$, and the symplectic form $\Omega$. The curvature has ghost number $0, \Omega$ has ghost number 2 . The deformation of type $(q, p)$ must be a polynomial of degree $p$ in the curvature (to match the cohomological degree) and of degree 0 in $\Omega$ (to have zero ghost number zero). But then it is impossible to contract the holomorphic indices to get a section of $\Lambda^{q} T_{X}$ with $q \geq 0$. Hence no quantum deformation of the classical result is possible.

The reduction of the RW model to the B-model has a further subtlety, which becomes apparent when one compares the partition function of the RW theory on $S^{1} \times \Sigma$ and the partition function of the B-model on $\Sigma$ [33]. When $\Sigma$ is a torus, they are both equal to the Euler characteristic of $X$. For $g(\Sigma)>1$ both partition functions vanish because of zero modes for the fermionic field $\chi$. But while the partition function of the B-model vanishes for $g(\Sigma)=0$, the partition function of the RW theory is equal to

$$
\int_{X} f\left(R_{X}\right),
$$

where $f\left(R_{X}\right)$ is a certain characteristic class of $X$. To explain this disagreement, note that the action of the B-model can be modified by a term

$$
\int_{\Sigma} R^{(2)} \mathcal{O},
$$

where $R^{(2)}$ is the curvature 2 -form representing $2 \pi$ times the Euler class of $\Sigma$ and $\mathcal{O}$ is any BRST-invariant local operator built from fields $\eta$ and $\theta$. We can choose $R^{(2)}$ so that it is concentrated at a single point on $\Sigma$. Then, when $\Sigma$ is a sphere, the partition function of the deformed B-model is the same as the expectation value of the operator $e^{4 \pi \mathcal{O}}$ in the undeformed B-model. Note further that observables in 
the B-model can be identified with forms on $X$ using the isomorphism $\Omega: T_{X} \rightarrow T_{X}^{*}$. Thus if we set $4 \pi \mathcal{O}=\log f\left(R_{X}\right)$, we can reproduce the partition function of the RW theory on $S^{1} \times \Sigma$. This curvaturedependent correction to the action of the B-model is again a quantum effect.

2.3. Complex lagrangian submanifolds. First we will look for BRSTinvariant boundary conditions for the sigma-model which do not involve additional degrees of freedom on the boundary.

Suppose the boundary is locally given by the equation $x^{3}=0$, and the interior of $M$ is $x^{3}>0$. Let us temporarily regard $x^{3}$ as the time coordinate. Then from the point of view of the canonical formalism, the space of fields and their conjugate momenta at $x^{3}=0$ is an infinite-dimensional symplectic manifold. A boundary condition then corresponds to a choice of a Lagrangian submanifold in this symplectic manifold. The equations defining the submanifold must be local in the $x^{1}, x^{2}$ directions, to ensure that the boundary condition is local. Upon quantization, this Lagrangian submanifold will become what is known as the boundary state. We also require the Lagrangian submanifold to be BRST-invariant.

On the other hand, one could also consider $x^{1}$ as the time coordinate. $^{2}$ Then the space of fields and their conjugate momenta on the hypersurface $x^{1}=0$ must have a well-defined symplectic structure. This provides an additional constraint on allowed boundary conditions.

To apply the canonical formalism, it is convenient to choose local coordinates in the collar neighborhood of $\partial M$ so that the metric on $\left.T_{M}\right|_{\partial M}$ has the form

$$
h_{\alpha \beta} d x^{\alpha} \otimes d x^{\beta}+d x^{3} \otimes d x^{3},
$$

where the indices $\alpha, \beta$ take values in the set $\{1,2\}$ and label the coordinates on the boundary. If we regard $x^{3}$ as the time coordinate, the momenta conjugate to $\phi^{I}$ and $\phi^{\bar{J}}$ are

$$
\begin{aligned}
& \pi_{\phi^{I}}=\frac{\partial \mathcal{L}^{\text {bulk }}}{\partial\left(\partial_{3} \phi^{I}\right)}=\operatorname{vol}_{\partial M} g_{I \bar{J}} \partial_{3} \phi^{\bar{J}}-\left.\frac{1}{2} \Omega_{K J} \Gamma_{I L}^{J} \chi^{K} \chi^{L}\right|_{\partial M} \\
& \pi_{\phi^{\bar{J}}}=\frac{\delta \mathcal{L}^{b u l k}}{\delta\left(\partial_{3} \phi^{\bar{J}}\right)}=\operatorname{vol}_{\partial M}\left(g_{I \bar{J}} \partial_{3} \phi^{I}-g_{I \bar{K}} \Gamma_{\bar{J} \bar{L}}^{\bar{K}} \chi_{3}^{I} \eta^{\bar{L}}\right)
\end{aligned}
$$

Here $v l_{\partial M}$ is the volume form of the induced metric:

$$
\operatorname{vol}_{\partial M}=\sqrt{h} d x^{1} d x^{2}, \quad h=\operatorname{det} h_{\alpha \beta} .
$$

\footnotetext{
${ }^{2}$ In this paper we will only consider the boundary conditions which do not break rotational invariance on the boundary, so one could equally well take any other direction along the boundary as the time.
} 
The symplectic form on the space of fields can be read off the boundary part of the variation of the bulk action

$$
-\int_{\partial M}\left[\pi_{\phi^{I}} \delta \phi^{I}+\pi_{\phi_{\bar{K}}} \delta \phi^{\bar{K}}-\operatorname{vol}_{\partial M} g_{I \bar{J}} \chi_{3}^{I} \delta \eta^{\bar{J}}-\frac{1}{2} \Omega_{I J} \chi^{I} \delta \chi^{J}\right] .
$$

This expression defines a 1-form on the space of fields whose exterior differential is the symplectic form.

The simplest boundary condition restricts the map $\phi: M \rightarrow X$ so that $\partial M$ is mapped to some submanifold $Y \subset X$. Such boundary conditions are called D-branes in the B-model. Every BRST-invariant boundary condition in the RW model gives, upon reduction on a circle, a BRST-invariant boundary condition in the B-model with the same target. It is well-known that $Y$ corresponds to a BRST-invariant Dbrane in the B-model only if it is a complex submanifold. Thus we may assume that $Y$ is complex.

We will now show that in the RW model $Y$ has to be a Lagrangian submanifold with respect to the holomorphic symplectic structure $\Omega_{I J}$. We may assume without loss of generality that locally $Y$ is given by the equations

$$
\phi^{I}=0, \quad I=2 n-m+1, \ldots, 2 n .
$$

Here $m$ is the complex codimension of $Y$. Let us adopt the convention that lower-case unprimed indices range from 1 to $2 n-m$, while lowercase primed indices range from $2 n-m+1$ to $2 n$. Then the equation of the submanifold $Y$ can be concisely written as

$$
\phi^{i^{\prime}}=0 \text {, }
$$

and BRST-invariance requires

$$
\eta^{\bar{i}^{\prime}}=0, \quad \chi_{\alpha}^{i^{\prime}}=0 .
$$

But since the Poisson bracket of the fields $\chi_{1}^{I}$ and $\chi_{2}^{I}$ is given by the inverse of the holomorphic symplectic form $\Omega$ (if we regard $x^{3}$ as the time coordinate), the last equation implies that $Y$ must be Lagrangian with respect to $\Omega$. One can express the boundary conditions on the fields $\eta^{\bar{J}}$ and $\chi_{\alpha}^{J}$ more invariantly by saying that the vector fields $\eta$ and $\chi_{\alpha}, \alpha=1,2$, are tangent to $Y$.

It is convenient from now on to assume that local coordinates $\phi^{i}, \phi^{i^{\prime}}$ have been chosen so that the symplectic form $\Omega$ is off-diagonal:

$$
\Omega=\Omega_{j k^{\prime}} d \phi^{j} d \phi^{k^{\prime}} \text {. }
$$

The matrix inverse to $\Omega_{j k^{\prime}}$ will be denoted $\Omega^{k^{\prime} j}$.

To complete the determination of boundary conditions, let us now regard $x^{1}$ as the time coordinate. With respect to $x^{1}$, the momentum 
conjugate to $\eta^{\bar{j}}$ is $g_{\bar{j} K} \sqrt{h} h^{1 \beta} \chi_{\beta}^{K}$. Since the fields $\chi_{\beta}^{j}, \beta=1,2$, are unconstrained on the boundary, the same should be true about $\eta^{\bar{j}}$. On the other hand, with respect to $x^{3}$ the field $\eta^{\bar{j}}$ is canonically conjugate to $g_{\bar{i} J} \chi_{3}^{J}$, so we need to require

$$
g_{\bar{i} J} \chi_{3}^{J}=0 .
$$

In other words, the vector field $\chi_{3}$ must be orthogonal to $T_{Y}$ with respect to the Hermitian metric. Finally, since the bosonic fields $\phi^{i}$ and $\phi^{\bar{i}}$ are unconstrained, we must require their conjugate momenta $(10,11)$ to vanish on the boundary:

$$
\pi_{\phi^{i}}=0, \quad \pi_{\phi^{\bar{i}}}=0 .
$$

One can verify that these boundary conditions are BRST-invariant. ${ }^{3}$

We would like to stress that boundary conditions involving nonLagrangian holomorphic submanifolds of $X$ are not allowed in the RW model, even though they correspond to valid topological branes in the B-model. The only way to avoid this restriction is to break explicitly the diffeomorphism invariance on the boundary.

So far the discussion was purely classical. We will see later that on the quantum level one has to require $Y$ to be a Calabi-Yau manifold to make sure that the measure in the path-integral is BRST-invariant.

2.4. Deformations of boundary conditions. We can find more general boundary conditions for the RW model by deforming the ones we constructed above. To deform a boundary condition, one adds a boundary term to the action and simultaneously modifies the BRST transformations of the fields by terms localized on the boundary, so that the whole action is BRST-invariant.

A systematic way to construct such a deformation is the descent procedure [40]. Let $\mathcal{O}$ be an even (i.e. bosonic) local topological observable on the boundary. Its descendants $\mathcal{O}^{(1)} \in \Omega^{1}(M)$ and $\mathcal{O}^{(2)} \in \Omega^{2}(M)$ are defined by

$$
d \mathcal{O}=\delta_{Q} \mathcal{O}^{(1)}+\ldots, \quad d \mathcal{O}^{(1)}=\delta_{Q} \mathcal{O}^{(2)}+\ldots
$$

\footnotetext{
${ }^{3}$ When checking the BRST-invariance of the first condition in (16) one has to use the equation of motion for $\chi_{3}^{i}$

$$
-g_{i \bar{J}} \operatorname{vol}_{\partial M} \nabla_{3} \eta^{\bar{J}}+\Omega_{i j^{\prime}}\left(\nabla \chi^{j^{\prime}}+\frac{1}{2} \mathcal{R}_{k l \bar{m}}^{j^{\prime}} \chi^{k} \chi^{l} \eta^{\bar{m}}\right)=0
$$
}

where all fields and the covariant derivative are restricted to the boundary. 
where dots denote terms proportional to equations of motion. Now consider adding to the action a boundary term

$$
S^{b u l k} \mapsto S^{b u l k}+\epsilon \int_{\partial M} \mathcal{O}^{(2)}
$$

where $\epsilon$ is a formal parameter. It follows from the definition of $\mathcal{O}^{(2)}$ that the modified action is BRST-invariant up to terms proportional to equations of motion. The latter can be removed by modifying the BRST transformations of bulk fields by terms proportional to the deltafunction localized on the boundary. In general, $\mathcal{O}^{(2)}$ is not invariant with respect to modified BRST transformations so one has to correct the boundary action further by terms of order $\epsilon^{2}$. Luckily, in all cases of interest to us this is not necessary, and the procedure stops here.

It is easy to see that local topological observables on the boundary are of the same form as in the bulk, i.e. they have the form

$$
\frac{1}{p !} \omega_{\bar{i}_{1} \ldots \bar{i}_{p}} \eta^{\bar{i}_{1}} \ldots \eta^{\bar{i}_{p}}
$$

for some $(0, p)$-form $\omega$ on $Y$. On the classical level the BRST operator again acts as the Dolbeault operator and therefore the space of boundary topological observables is isomorphic to

$$
\oplus_{p=0}^{n} H^{p}\left(\mathcal{O}_{Y}\right)
$$

The $\mathbb{Z}_{2}$-grading is given by $p \bmod 2$. In what follows we will denote $p \bmod 2$ by $\hat{p}$. Thus the even subspace of $H^{\bullet}\left(\mathcal{O}_{Y}\right)$ will be denoted $H^{\hat{0}}\left(\mathcal{O}_{Y}\right)$, and the odd subspace will be denoted $H^{\hat{1}}\left(\mathcal{O}_{Y}\right)$. On the classical level, the algebra structure is given by the usual exterior product. We will show later that the algebra of boundary observables is not modified by quantum corrections.

Infinitesimal deformations of the boundary condition are parameterized by $H^{\hat{0}}\left(\mathcal{O}_{Y}\right)$. An element of this space can be represented by a $\bar{\partial}$-closed inhomogeneous form $W$ of even degree. The corresponding observable can be thought of as an even function $W(\phi, \eta)$ of bosonic variables $\phi^{i}, \phi^{\bar{i}}$ and fermionic variables $\eta^{\bar{i}}$ satisfying

$$
\eta^{\bar{i}} \frac{\partial W(\phi, \eta)}{\partial \phi^{\bar{i}}}=0
$$

More invariantly, one can say that $W$ is a function on the odd tangent bundle $\Pi \bar{T}_{Y}$ annihilated by the odd vector field

$$
\bar{\partial}=\eta^{\bar{i}} \frac{\partial}{\partial \phi^{i}} .
$$


We will call this function a curving on $Y$. The origin of this terminology will become clear later.

Next we construct the descendants of $W$. Let $\partial_{i}$ and $\partial_{\bar{i}}$ denote partial derivatives with respect to $\phi^{i}$ and $\phi^{\bar{i}}$ respectively. We first note that the equation (19) implies

$$
\partial_{\bar{j}} W=\eta^{\bar{i}} \partial_{\bar{i}} \frac{\partial W}{\partial \eta^{\bar{j}}}=\delta_{Q} \frac{\partial W}{\partial \eta^{\bar{j}}}, \quad \partial_{\bar{i}} \frac{\partial W}{\partial \eta^{\bar{j}}}-\partial_{\bar{j}} \frac{\partial W}{\partial \eta^{\bar{i}}}=\delta_{Q} \frac{\partial^{2} W}{\partial \eta^{\bar{i}} \partial \eta^{\bar{j}}} .
$$

Using these relations, we find

$$
W^{(1)}=\chi^{i} \partial_{i} W+d \phi^{i} \frac{\partial W}{\partial \eta^{\bar{i}}},
$$

$$
W^{(2)}=\frac{1}{2} \chi^{i} \chi^{j} \hat{\nabla}_{i} \partial_{j} W+\chi^{i} d \phi^{\bar{j}} \partial_{i} \frac{\partial W}{\partial \eta^{\bar{j}}}+\frac{1}{2} d \phi^{\bar{i}} d \phi^{\bar{j}} \frac{\partial^{2} W}{\partial \eta^{\bar{i}} \partial \eta^{\bar{j}}}-\operatorname{vol}_{\partial M} \Omega^{k^{\prime} i} A_{k^{\prime}}^{K} \partial_{i} W g_{K \bar{J}} \partial_{3} \phi^{\bar{J}} .
$$

Here $\hat{\nabla}$ is the covariant differential with respect to the induced metric on $Y$ and $A_{k^{\prime}}^{K}$ is defined by

$$
A_{k^{\prime}}^{i^{\prime}}=\delta_{k^{\prime}}^{i^{\prime}}, \quad A_{k^{\prime}}^{i}=-g_{k^{\prime}} \hat{g}^{\hat{g}^{j}}
$$

where $\hat{g}^{\bar{j} i}$ is the matrix inverse to $g_{i \bar{j}}$. The matrix $A_{k^{\prime}}^{K}$ represents the unique bundle map $A:\left.N_{Y} \rightarrow T_{X}\right|_{Y}$ which splits the exact sequence

$$
\left.0 \rightarrow T_{Y} \rightarrow T_{X}\right|_{Y} \rightarrow N_{Y} \rightarrow 0 \text {. }
$$

and identifies $N_{Y}$ with the orthogonal complement of $T_{Y}$. The splitting $A$ is nonholomorphic, and in general no holomorphic splitting exists; some consequences of this are described in the next subsection.

The descendants satisfy

$$
\delta_{Q} W^{(1)}=d W, \quad \delta_{Q} W^{(2)}=d W^{(1)}+\Omega^{k^{\prime} i} \partial_{i} W A_{k^{\prime}}^{K} \frac{\delta S^{b u l k}}{\delta \chi_{3}^{K}} .
$$

We define the boundary action as

$$
S^{b r y}=\int_{\partial M} W^{(2)}
$$

and modify the BRST transformation for $\chi_{3}$ by a boundary term:

$$
\delta_{Q, W} \chi_{3}^{K}=\partial_{3} \phi^{K}-\delta\left(x_{3}\right) A_{k^{\prime}}^{K} \Omega^{k^{\prime} i} \partial_{i} W
$$

Then the total action is BRST-invariant up to a total derivative on the boundary:

$$
\delta_{Q, W}\left(S^{b u l k}+S^{b r y}\right)=\int_{\partial M} d W^{(1)}
$$


It is instructive to look at a couple of special cases. If $W$ is a degree- 0 form, then it is simply a holomorphic function on $Y$. The boundary action simplifies to

$$
S^{b r y}=\int_{\partial M}\left(\frac{1}{2} \hat{\nabla}_{i} \partial_{j} W \chi^{i} \chi^{j}-\operatorname{vol}_{\partial M} \Omega^{k^{\prime} i} \partial_{i} W A_{k^{\prime}}^{K} g_{K \bar{J}} \partial_{3} \phi^{\bar{J}}\right)
$$

The first term in this action is reminiscent of the Landau-Ginzburg deformation of the B-model (see Appendix), with $W$ playing the role of the superpotential. We will see later on that this is more than a mere similarity.

If $W$ has degree 2 , then it corresponds to a $\bar{\partial}$-closed $(0,2)$ form $B_{\overline{i j}}$ on $Y$ :

$$
W=\frac{1}{2} B_{\overline{i j}} \eta^{\bar{i}} \eta^{\bar{j}}
$$

The corresponding boundary action is similar to the deformation of the B-model by a $(0,2)$ B-field. To make the similarity more obvious, let us recall that $X$ and therefore $Y$ are Kähler manifolds. If we assume in addition that $Y$ is compact, then the Dolbeault cohomology class of $B$ contains a closed 2-form, and therefore we may assume without loss of generality that $B$ is closed. Then the boundary action simplifies to

$$
S^{b r y}=\int_{\partial M} \phi^{*} B
$$

In the context of $2 \mathrm{~d}$ sigma-models such a term in the bulk action is known as the B-field. In 3d sigma-model it deforms the boundary action instead. Note that in this case no modification of the BRST transformation is necessary.

Finally, we need to consider deformations of the boundary action corresponding to geometric deformations of the Lagrangian submanifold. An infinitesimal deformation of $Y$ corresponds to a holomorphic section $\xi$ of the normal bundle $N_{Y}$. The symplectic form identifies $N_{Y}$ with $T_{Y}^{*}$; the requirement that the deformed submanifold be Lagrangian is equivalent to $\partial(\Omega \xi)=0$. Thus infinitesimal deformations are in one-to-one correspondence with closed holomorphic 1-forms on $Y$. Locally, such a form can be integrated to a holomorphic function $W$ on $Y$. This suggests that an infinitesimal deformation of the Lagrangian submanifold corresponding to a 1-form $\alpha$ can be described by the boundary action (25) where $\partial W$ is replaced by $\alpha$ times a constant factor.

To prove this statement, note that the variation of the boundary action contains a peculiar term

$$
-\int_{\partial M} \operatorname{vol}_{\partial M} \Omega^{k^{\prime} i} \partial_{i} W A_{k^{\prime}}^{K} g_{K \bar{J}} \delta\left(\partial_{3} \phi^{\bar{J}}\right) .
$$


To cancel such a term by a boundary term in the variation of the bulk action, we must assume that the field $\phi^{K}$ has a step-like discontinuity on the boundary:

$$
\lim _{x_{3} \rightarrow 0+} \phi^{K}\left(x_{1}, x_{2}, x_{3}\right)=\phi^{K}\left(x_{1}, x_{2}, 0\right)+A_{k^{\prime}}^{K} \Omega^{k^{\prime} i} \partial_{i} W .
$$

Therefore deforming the boundary action by $W$ is equivalent to deforming $Y$ along a normal vector field

$$
\xi^{k^{\prime}}=\Omega^{k^{\prime} i} \partial_{i} W
$$

We can describe this result a bit differently by introducing local Darboux coordinates $q^{i}, p_{i}, i=1, \ldots, n$, so that the symplectic form is $\Omega=d q^{i} d p_{i}$ and the undeformed Lagrangian is given by the equations $p_{i}=0$. Given a holomorphic function $W$ on $Y$, we define a deformed Lagrangian submanifold $\tilde{Y}$ by

$$
p_{i}=\frac{\partial W}{\partial q^{i}}, \quad i=1, \ldots, n .
$$

In symplectic geometry, the function $W$ is known as the generating function of the Lagrangian $\tilde{Y}$. We have shown above that on the infinitesimal level the boundary condition corresponding to $\tilde{Y}$ is equivalent to the deformation of $Y$ by the generating function $W$.

It is tempting to extend this result from infinitesimal to finite deformations and identify the superpotential $W$ with the generating function of the deformed Lagrangian submanifold $\tilde{Y}$. But such an identification appears problematic because the generating function depends on the choice of Darboux coordinates, while $W$ does not. The likely resolution is that the superpotential deformation is not well-defined beyond leading order in $W$, and the additional choices one has to make to define it amount to a choice of local Darboux coordinates. Such ambiguities in perturbation theory typically arise from short-distance divergences which require renormalization.

2.4.1. Obstructions. While classically adding a descendant 2-form to the boundary action preserves BRST-invariance, this is not necessarily true on the quantum level beyond first order in the deformation. Consequently, at second order in $W$ one may have an obstruction to deformation. An analogous phenomenon is well-known in the context of B-branes: deformations of complex submanifolds, holomorphic vector bundles or more general objects of the derived category of coherent sheaves may be obstructed. A less-known case where such obstructions occur is the B-model itself, in the case when the target manifold is not compact and Kähler. (One can show that the deformation theory of compact Kähler Calabi-Yau manifolds is unobstructed [7, 35, 34]). We 
will see below that the situation for boundary deformations in the RW model is similar to that in the B-model: if $Y$ is compact and Kähler, the deformation theory is unobstructed, while in general there are obstructions.

As shown above, geometric deformations of $Y$ are essentially a special case of deformations by means of a curving $W$. A geometric counterpart of our result is a theorem proved by C. Voisin [37] which implies that deformations of complex Lagrangian submanifolds in a complex symplectic manifold are unobstructed in the compact Kähler case.

One difference between deformations of the B-model and boundary deformations of the RW model is that in the latter case obstructions arise from the external geometry of $Y$ in $X$. It is well-known that in the real case the formal neighborhood of a submanifold is isomorphic to the total space of its normal bundle. In the complex case this is no longer the case. To describe the difference, consider again a short exact sequence of sheaves (23). It defines a class $[\beta] \in \operatorname{Ext}^{1}\left(N_{Y}, T_{Y}\right)=$ $H^{1}\left(T_{Y} \otimes N_{Y}^{*}\right)$ which measures the failure of the exact sequence to split holomorphically. This cohomology class also describes the leading deviation of the complex geometry of the neighborhood of $Y$ in $X$ from the geometry of the total space of the holomorphic vector bundle $N_{Y}$. To be more concrete, let $x^{i}, i=1, \ldots, \operatorname{dim}_{\mathbb{C}} Y$ denote local complex coordinates on $Y$ and $z^{\alpha}, \alpha=1, \ldots, \operatorname{codim}_{\mathbb{C}} Y$, denote complex linear coordinates on the fibers of $N_{Y}$ with respect to some local trivialization $e_{\alpha}$. Then to leading order in $z$ the difference between the $\bar{\partial}$ operator on the neighborhood of $Y$ and the $\bar{\partial}$-operator on the total space of $N_{Y}$ is

$$
z^{\alpha} \beta_{\alpha \bar{k}}^{i} d x^{\bar{k}} \partial_{i}
$$

where

$$
\beta_{\alpha \bar{k}}^{i} e^{\alpha} d x^{\bar{k}} \partial_{i}
$$

is a Dolbeault representative of $[\beta]$. From the differential-geometric viewpoint, it can be represented by the second fundamental form of $Y$.

An equivalent description of this class using Cech cohomology goes as follows. Let us choose local coordinates $x^{i}, z^{\alpha}$ on a neighborhood $\hat{Y}$ of $Y$ so that $Y$ is given by $z^{\alpha}=0, \alpha=1, \operatorname{codim}_{\mathbb{C}} Y$. The normal bundle of $Y$ is spanned by partial derivatives along $z^{\alpha}$ at $z^{\alpha}=0$. On an overlap of two charts we have two sets of coordinates $x^{i}, z^{\alpha}$ and $\tilde{x}^{i}, \tilde{z}^{\alpha}$. We consider a section of $\operatorname{Hom}\left(N_{Y}, T_{Y}\right)$ given by

$$
\frac{\partial \tilde{x}^{i}}{\partial z^{\alpha}}\left(d z^{\alpha} \otimes \frac{\partial}{\partial \tilde{x}^{i}}\right) .
$$


It defines a cocycle $\beta$ with values in $\operatorname{Hom}\left(N_{Y}, T_{Y}\right)$. If $\hat{Y}$ happens to be isomorphic to $N_{Y}$, one can choose the coordinates $x^{i}$ on all charts so that on double overlaps $\tilde{x}^{i}$ depends only on $x^{i}$ and not on $z^{\alpha}$; then the cocycle $\beta$ is trivial.

In the case when $Y$ is a complex Lagrangian submanifold of a complex symplectic manifold $X, N_{Y}$ is isomorphic to $T_{Y}^{*}$, and the class [ $\left.\beta\right]$ can be regarded as an element of $H^{1}\left(T_{Y} \otimes T_{Y}\right)$. Moreover, an easy computation in holomorphic Darboux coordinates shows that $[\beta]$ belongs to $H^{1}\left(\operatorname{Sym}^{2} T_{Y}\right)$, i.e. one can assume that $\beta_{\bar{k}}^{i j}=\beta_{\bar{k}}^{j i}$. A particular Dolbeault representative of $[\beta]$ is given by

$$
\beta_{\bar{k}}^{i j}=\Omega^{k^{\prime} i} \partial_{\bar{k}} A_{k^{\prime}}^{j}
$$

Note that $A_{k^{\prime}}^{j}$ does not transform as a tensor, but its $\bar{\partial}$-differential does, so the form $\beta$ is not necessarily exact.

The description of the formal neighborhood of $Y$ can be extended to all orders in the normal coordinates. Let us choose local Darboux coordinates $x^{i}, p_{i}$ such that $Y$ is given by $p_{i}=0$. In such coordinates the transition functions must arise from a generating function on an overlap of two coordinate charts, which upon Taylor expansion in powers of $p_{i}$ can be regarded as a holomorphic section of

$$
\oplus_{\ell} \operatorname{Sym}^{\ell} T_{Y} \text {. }
$$

In addition, the Taylor expansion must start with quadratic terms, so that in new coordinates $Y$ is still given by the equation $p_{i}=0$. Thus the transition functions can be encoded in an element of

$$
\oplus_{\ell \geq 2} H^{1}\left(\operatorname{Sym}^{\ell} T_{Y}\right) \text {. }
$$

We will denote by $\left[\beta^{(\ell)}\right]$ the $\ell^{\text {th }}$ homogeneous component of this class. The class $[\beta]$ we considered before is the same as $\left[\beta^{(2)}\right]$. Note that multiplying the symplectic form $\Omega$ by a factor $\lambda^{2}$ requires multiplying the coordinates $p_{i}$ and the generating function by the same factor. Hence the class $\beta^{(\ell)}$ has ghost number $2-2 \ell$. This will be useful later on.

Let us now explain how the obstruction arises at second order in $W$. The key observation is that adding $W$ to the action modifies the BRST transformation not only of $\chi_{3}^{K}$, but also of $\chi_{1,2}^{i}$ and $\phi^{i}$. Indeed, one can easily see that unless we modify the BRST transformation of $\phi^{i}$, the BRST transformation (24) does not satisfy $\delta_{Q, W}^{2} \chi_{3}=0$. We can rectify this by letting

$$
\delta_{Q, W} \phi^{i}\left(x^{3}\right)=-\theta\left(-x^{3}\right) \beta_{\bar{k}}^{i j} \partial_{j} W \eta^{\bar{k}}
$$


where $\theta(x)$ is a unit step-function, i.e. $\theta(x)=1$ if $x \geq 0$ and $\theta(x)=0$ if $x<0$. Then

$$
\partial_{3} \delta_{Q, W} \phi^{i}=\delta\left(x^{3}\right) \beta_{\bar{k}}^{i j} \partial_{j} W \eta^{\bar{k}}
$$

and we see that $\delta_{Q, W}^{2} \chi_{3}=0$. Alternatively, we can derive this formula from (26), (28) and

$$
\lim _{x^{3} \rightarrow 0+} \delta_{Q, W} \phi^{i}\left(x^{1}, x^{2}, x^{3}\right)=0 .
$$

Since $\Omega^{k^{\prime} i}$ is implicitly proportional to the Planck constant, we should regard this as the leading quantum correction to the BRST transformation. Similarly, one can determine quantum corrections to the BRST variations of the fields $\chi_{1,2}^{i}$.

Note that the variation of the bulk action is unaffected by the modifications in the BRST transformations of the fields $\phi^{i}, \chi_{1,2}^{i}$, because these modifications are concentrated on the boundary and the momenta conjugate to these fields vanish on the boundary. But the curving $W$ is no longer BRST-invariant:

$$
\delta_{Q, W} W=-\beta_{\bar{k}}^{i j} \eta^{\bar{k}} \partial_{i} W \partial_{j} W .
$$

Similarly, $W^{(2)}$ is no longer BRST-invariant up to total derivatives and the equation of motion for $\chi_{3}^{K}$. To rectify this, let us replace $W$ with a power series in the Planck constant $W=W_{0}+W_{1}+\ldots$, where the term $W_{n}$ is of order $\hbar^{n}$. Then BRST invariance requires $W_{0}$ to satisfy (19), while $W_{1}$ must satisfy

$$
\bar{\partial} W_{1}=\beta\left(d W_{0}, d W_{0}\right) .
$$

This equation has solutions if and only if the cohomology class

$$
\left[\beta\left(d W_{0}, d W_{0}\right)\right] \in H^{\hat{1}}\left(\mathcal{O}_{Y}\right)
$$

is trivial. This is the leading obstruction to the boundary deformation corresponding to $W_{0}$.

An alternative derivation of the same result goes as follows. Consider the path-integral with a boundary insertion

$$
W(0) \int_{\partial M} W^{(2)}
$$

Naively, since the BRST-variation of $W^{(2)}$ is a total derivative, and $W$ is BRST-invariant, this expression is also BRST-invariant. But because of possible short-distance singularities when the insertion point of $W^{(2)}$ approaches 0 , one should be more careful. Let $D_{\epsilon} \subset \partial M$ be a small 
disk centered at 0 . One can define the above product as the limit

$$
\lim _{\epsilon \rightarrow 0} W(0) \int_{\partial M \backslash D_{\epsilon}} W^{(2)} .
$$

The BRST-variation $\delta_{Q}$ of this expression is

$$
\oint W^{(1)} W(0)
$$

where integration is over a small circle on $\partial M$ centered at 0 . If the operator product of $W^{(1)}$ and $W$ is singular, this expression may be nonzero. In fact, as shown in appendix $\mathrm{C}$, there is a singularity in the boundary operator product of the fields $\left.\chi^{i}\right|_{\partial M}$ and $\delta \phi^{j}$, where $\delta \phi^{j}$ is a deviation of $\phi^{j}$ from its classical background value. If $z$ is a local complex coordinate on $\partial M$, the singularity in the OPE is

$$
\begin{aligned}
\chi_{z}^{i}(z, \bar{z}) \delta \phi^{j}(0) & \sim \frac{C}{z} \beta_{\bar{k}}^{i j} \eta^{\bar{k}}+\ldots \\
\chi_{\bar{z}}^{i}(z, \bar{z}) \delta \phi^{j}(0) & \sim-\frac{C}{\bar{z}} \beta_{\bar{k}}^{i j} \eta^{\bar{k}}+\ldots
\end{aligned}
$$

where $C$ is a constant. Therefore the operator product (31) is proportional to

$$
\beta_{\bar{k}}^{i j} \eta^{\bar{k}} \partial_{i} W \partial_{j} W
$$

If this form vanishes identically, there is no obstruction at second order in $W$. More generally, if the form does not vanish but is a trivial class in $\bar{\partial}$-cohomology, we can restore BRST-invariance at second order by writing $W=W_{0}+W_{1}+\ldots$ as above and requiring $W_{1}$ to satisfy (29).

If $Y$ is compact and Kähler, we can choose a representative of the Dolbeault cohomology class $\left[W_{0}\right]$ which is annihilated by $\partial$ (namely, the harmonic representative). This implies that the Dolbeault cohomology class of $\partial W_{0}$ is trivial, and therefore the obstruction (34) is trivial.

Let us compare this physical obstruction to the one arising from deformation theory of complex Lagrangian submanifolds. As shown by Kodaira [25], all obstructions to deforming a complex submanifold $Y$ of a complex manifold $X$ take values in $H^{1}\left(N_{Y}\right)$. In the case when $Y$ is Lagrangian, we have $N_{Y} \simeq T_{Y}^{*}$, and obstructions take values in $H^{1}\left(\Omega_{Y}^{1}\right)$. If we want the deformed manifold to be Lagrangian, we must require that the holomorphic 1-form $\alpha$ on $Y$ corresponding to $\xi$ is $\partial$ closed. Then a somewhat lengthy computation shows that the first obstruction is the Dolbeault cohomology class of

$$
\partial(\beta(\alpha \otimes \alpha)) \in \Omega_{Y}^{1,1} .
$$


In the situation considered above, $\alpha=\partial W$, where $W$ is a holomorphic function, and we see that the obstruction to deforming the submanifold is $\partial$ of (30). In the compact Kähler case, the obstruction vanishes, in agreement with [37]. Note that the geometric problem of deforming a complex Lagrangian submanifold is a special case of the physical problem of deforming the boundary condition in the RW theory: in the former case $\partial W$ is a closed holomorphic 1-form, while in the latter case it is a $\partial$ and $\bar{\partial}$-closed form of type $(1, \hat{0})$.

Deforming $Y$ as a submanifold of $X$ may deform the complex structure of $Y$. In general, an infinitesimal deformation of the complex structure of $Y$ is described by a class $[\mu] \in H^{1}\left(T_{Y}\right)$. Its Dolbeault representative $\mu$ is called a Beltrami differential. Given a Beltrami differential, we can deform the $\bar{\partial}$ operator on $Y$ by letting

$$
\bar{\partial}^{\text {new }}=\bar{\partial}-\mu(\partial) \text {. }
$$

It is easy to see that the Beltrami differential $\mu$ corresponding to a normal vector field $\xi$ on $Y$ is

$$
\mu=\beta(\xi),
$$

where we regard $\beta$ a section of $\operatorname{Hom}\left(N_{Y}, T_{Y}\right) \otimes \Omega_{Y}^{0,1}$. Therefore the deformation of the $\bar{\partial}$ operator on $Y$ corresponding to a curving $W$ is

$$
\bar{\partial}^{\text {new }}=\bar{\partial}-\beta_{\bar{k}}^{i j} d x^{\bar{k}} \partial_{j} W \partial_{i} .
$$

The corresponding modification of the BRST transformation for the fields $\phi^{i}$ and $\phi^{\bar{i}}$ is

$$
\delta_{Q, W} \phi^{\bar{i}}=\eta^{\bar{i}}, \quad \delta_{Q, W} \phi^{i}=-\beta_{\bar{k}}^{i j} \partial_{j} W \eta^{\bar{k}} .
$$

This is precisely what we found above for the BRST transformation of the fields $\phi^{\bar{i}}, \phi^{i}$ evaluated at $x^{3}=0$.

\subsection{Calabi-Yau fibrations.}

2.5.1. Boundary degrees of freedom. In the case of a two-dimensional sigma-model, an important class of boundary conditions arises from vector bundles on the target space or its submanifolds. These boundary conditions involve extra degrees of freedom on the boundary and therefore are not really conditions but boundary terms in the action which describe interactions between bulk and boundary degrees of freedom.

A systematic way to deduce the existence of such boundary conditions in the $2 \mathrm{~d}$ sigma-model is to consider first boundary degrees of freedom completely decoupled from the bulk. Such degrees of freedom are described by ordinary quantum mechanics; in the topological case, 
this means that states are described by elements of a finite-dimensional vector space, or a graded vector space, while observables are arbitrary linear operators on this space. If the vector space has dimension $n$, this corresponds to a trivial rank- $n$ vector bundle on the brane. Next one considers deformations which introduce interactions between bulk and boundary degrees of freedom. It turns out that the most general such deformation corresponds to a complex of vector bundles over the brane; in the B-model BRST-invariance requires the vector bundles to be holomorphic and the differential to commute with the $\bar{\partial}$ operator. This line of thought eventually leads one to identify D-branes in the B-model with objects of the bounded derived category of coherent sheaves.

Let us apply the same strategy to the RW model. If the boundary degrees of freedom are decoupled from the bulk, they must be described by a two-dimensional topological field theory. Since the bulk theory resembles the B-model, it is very natural to consider a B-model with a Calabi-Yau target $Z$ living on the boundary of $M$. Recall that in general a deformation of a B-model is described not in terms of classical geometry but by an $A_{\infty}$ Calabi-Yau category $[26,10]$. To keep the discussion concrete, we will limit ourselves to a particular class of deformations, namely the ones involving even $\bar{\partial}$-closed forms of type $(0, p)$. As discussed in the appendix, the case $p=0$ corresponds to a superpotential deformation, while the case $p=2$ corresponds to a B-field. The advantage of this class of topological field theories is that they can be described by a relatively simple explicit action. More general deformations involving forms with values in polyvector fields are possible, but the corresponding deformations are difficult to write down in a closed form [42].

Calabi-Yau sigma-model with target $Z$ deformed by a holomorphic function $W$ on $Z$ is known as a Landau-Ginzburg model. Of course, in order for a nonconstant superpotential $W$ to exist, $Z$ must be noncompact. This does not cause any problems provided the critical set of $W$ is compact, and the resulting topological field theory is very similar to a B-model with a compact target space. There is one important distinction though: while the ordinary B-model is usually regarded as $\mathbb{Z}$-graded (the grading is provided by the vector $\mathrm{R}$-charge), the LandauGinzburg model is only $\mathbb{Z}_{2}$-graded. ${ }^{4}$ For this reason, it is natural to

\footnotetext{
${ }^{4}$ Note that if one sets $W=0$ in the Landau-Ginzburg model, one recovers not the usual B-model with a noncompact target $Z$, but its $\mathbb{Z}_{2}$-graded version. In particular, the resulting category of B-branes is not the bounded derived category of $Z$, but the derived category of 2-periodic complexes of $\mathcal{O}_{Z}$-modules with coherent cohomology.
} 
consider, along with the superpotential deformation, deformations by arbitrary even $(0, p)$ forms, $p>0$. It is convenient to combine all these deformations into an inhomogeneous even form on $Z$; BRST-invariance requires it to be $\bar{\partial}$-closed. We will call such a form the curving, and the corresponding 2d TFT the curved B-model; its properties are discussed in section $B$. Since the RW model is $\mathbb{Z}_{2}$-graded, it is very natural to consider coupling it to a curved B-model on the boundary.

We are thus led to consider boundary degrees of freedom corresponding to a curved B-model with a Calabi-Yau target $Z$. Allowing interactions between bulk and boundary degrees of freedom amounts to deforming the boundary action by descendants of local operators of the form

$$
W_{Y} \cdot W_{Z}
$$

where $W_{Y} \in \oplus_{r} H^{r}\left(\mathcal{O}_{Y}\right)$ is a boundary topological observable constructed from the restrictions of bulk degrees of freedom, and $W_{Z}$ is a topological observable constructed from boundary degrees of freedom. For example, if the boundary degrees of freedom are described by the ordinary B-model with a Calabi-Yau target $Z, W_{Z}$ is an element of $\oplus_{p, q} H^{p}\left(\Lambda^{q} T_{Z}\right)$. In general, the only constraint on the local operator (35) is that it must be even. One can think of such a deformation as an even element of the vector space

$$
\oplus_{s, r} H^{s}\left(\Lambda^{r} T_{\mathcal{Y}}^{v}\right)
$$

where $\mathcal{Y}=Y \times Z$ is regarded as a trivial fibration with base $Y$ and fiber $Z$, and $T_{\mathcal{Y}}^{v}$ is the vertical tangent bundle of $\mathcal{Y}$, i.e. the bundle of vector fields tangent to the fibers.

In particular, elements of the subspace

$$
H^{1}\left(T_{\mathcal{Y}}^{v}\right)
$$

correspond to infinitesimal deformations of the product $Y \times Z$ into a general complex fibration with base $Y$. We conclude that in general boundary degrees of freedom can be thought of as taking values in the fiber of such a fibration.

We will begin by constructing a BRST-invariant boundary action for such degrees of freedom. Then we will consider a class of deformations of the boundary action parameterized by the subspace

$$
\oplus_{s} H^{s}\left(\mathcal{O}_{\mathcal{Y}}\right)
$$

More general deformations corresponding to graded components of the vector space (36) with $r>0$ also exist, but are more difficult to describe in a closed form. 
2.5.2. The Ehresmann connection. To write down a boundary action which is invariant under a change of local coordinates on $\mathcal{Y}$, we need to discuss connections on complex fibrations. Let the boundary $\partial M$ be mapped to the total space of the fibration $p: \mathcal{Y} \rightarrow Y$. The map $p$ is assumed to be holomorphic, and a fiber of $p$ is a Calabi-Yau m-fold. As before, $Y$ is a complex Lagrangian submanifold of a hyper-Kähler target space $X$. Let

$$
\phi^{A}, \quad A=1, \ldots, n+m,
$$

be local complex coordinates on the fibration $\mathcal{Y} \rightarrow Y$ so that

$$
\phi^{i}, \quad i=1, \ldots, n,
$$

are local complex coordinates on the base $Y$, while

$$
\phi^{a}, \quad a=n+1, \ldots, n+m
$$

are local complex coordinates on the fiber.

A vector field on $\mathcal{Y}$ is called vertical if it is tangent to the fibers of $p$, i.e. it belongs to the kernel of $d p$. In local complex coordinates, a vertical $(1,0)$ vector field $\xi$ has the form

$$
\xi^{a} \frac{\partial}{\partial \phi^{a}}
$$

where the functions $\xi^{a}$ may depend on both base and fiber coordinates. Vertical vector fields form a smooth complex subbundle of $T_{\mathcal{Y}}$ which we denote $T_{\mathcal{Y}}^{v}$. The quotient vector bundle $T_{\mathcal{Y}} / T_{\mathcal{Y}}^{v}$ will be denoted $T_{\mathcal{Y}}^{h}$; it is naturally isomorphic to $p^{*} T_{Y}$.

A 1-form on $\mathcal{Y}$ is called horizontal if it annihilates vertical vector fields. In local coordinates a horizontal $(1,0)$-form has the form

$$
\alpha_{i} d \phi^{i} .
$$

The bundle of horizontal $(1,0)$-forms is a subbundle of $T_{\mathcal{Y}}^{*}$ which we denote $T_{\mathcal{Y}}^{* h}$.

An Ehresmann connection on a smooth fibration $\mathcal{Y}$ is a splitting of the tangent bundle $T_{\mathcal{Y}}$ into a sum

$$
T_{\mathcal{Y}}^{v} \oplus T_{\mathcal{Y}}^{h}
$$

We will assume that this splitting is complex, i.e. respects the complex structure of the bundles. Such a splitting always exists. We do not assume that the splitting is holomorphic, since a holomorphic splitting rarely exists. In local coordinates the splitting $\xi \mapsto\left(\xi_{v}, \xi_{h}\right)$ is described by

$$
\xi_{v}=\left(\xi^{a}-V_{i}^{a} \xi^{i}\right) \frac{\partial}{\partial \phi^{a}}, \quad \xi_{h}=\xi^{i} \frac{\partial}{\partial \phi^{i}},
$$


and similarly for vector fields of type $(0,1)$. Given an Ehresmann connection $\left(V_{i}^{a}, V_{\bar{i}}^{\bar{a}}\right)$, we can define a horizontal vector field on $\mathcal{Y}$ as a vector field which belongs to $T_{\mathcal{Y}}^{h}$. Any vector field $\psi$ on the base $Y$ is lifted uniquely to a horizontal vector field $\psi^{h}$ on $\mathcal{Y}$.

We define the covariant derivative of a section

$$
s: Y \rightarrow \mathcal{Y}, \quad s:\left(\phi^{i}, \phi^{\bar{i}}\right) \mapsto s^{a}\left(\phi^{i}, \phi^{\bar{i}}\right)
$$

along a vector field $\xi=\left(\xi^{i}, \xi^{\bar{i}}\right)$ on $Y$ by local formulas

$$
\left(\nabla_{\xi} s\right)^{a}=\xi^{i} \nabla_{i} s^{a}+\xi^{\bar{i}} \nabla_{\bar{i}} s^{a}=\xi^{i}\left(\partial_{i} s^{a}-V_{i}^{a}\right)+\xi^{\bar{i}} \partial_{\bar{i}} s^{a} .
$$

Note that the covariant derivative in the $(0,1)$ direction coincides with the ordinary derivative. This happens because we assumed that the Ehresmann connection is compatible with the complex structure of $T_{\mathcal{Y}}$ and as a consequence the mixed component of the connection $V_{\bar{i}}^{a}$ is zero.

In the theory of Ehresmann connections, the Lie algebra of vertical vector fields plays the same role as the Lie algebra of the gauge group in Yang-Mills theory. Thus we should think of $V_{i}^{a} d \phi^{i}$ as components of a horizontal 1-form on $\mathcal{Y}$ with values in vertical vector fields. Similarly, the curvature of the Ehresmann connection is a horizontal 2-form with values in vertical vector fields. In our case, it only has $(1,1)$ and $(2,0)$ parts:

$$
\begin{aligned}
& \mathcal{R}_{i \bar{j}}^{a}=\partial_{\bar{j}} V_{i}^{a}+V_{\bar{j}}^{\bar{b}} \partial_{\bar{b}} V_{i}^{a} \\
& \mathcal{R}_{i j}^{a}=\partial_{j} V_{i}^{a}-\partial_{i} V_{j}^{a}+V_{j}^{b} \partial_{b} V_{i}^{a}-V_{i}^{b} \partial_{b} V_{j}^{a} .
\end{aligned}
$$

2.5.3. An affine connection on the fibration. To construct a boundary action, we will need a Kähler metric on the fibers of $\mathcal{Y}$ (actually, a Hermitian one would suffice) and an affine torsion-free connection on the vector bundle $T_{\mathcal{Y}}$ compatible with the complex structure. In principle, any such connection would do, and the topological correlators can be shown not to depend on the choice. But to minimize the geometric input, it is convenient to construct the affine connection starting from the fiberwise Kähler metric, the Kähler metric on the base $Y$, and an Ehresmann connection on $\mathcal{Y}$. Note that we do not require the total space of the fibration to be Kähler: that would be an unnecessary and rather strong restriction.

The vector bundles $T_{\mathcal{Y}}^{v}$ and $T_{\mathcal{Y}}^{h}$ have natural connections. The connection on $T_{\mathcal{Y}}^{h}$ is a pull-back of the Levi-Civita connection of the induced metric on $Y$. The corresponding Christoffel symbols are

$$
\hat{\Gamma}_{j k}^{i}=\Gamma_{j k}^{i}-A_{i^{\prime}}^{i} \Gamma_{j k}^{i^{\prime}},
$$


where $A_{i^{\prime}}^{i}$ is defined by (22). Thus if $\xi$ is a horizontal vector field and $\zeta$ is an arbitrary vector field, we define

$$
\nabla_{\zeta} \xi^{i}=\zeta^{A} \partial_{A} \xi^{i}+\hat{\Gamma}_{j k}^{i} \zeta^{j} \xi^{k}
$$

In order to describe the affine connection on $T_{\mathcal{Y}}^{v}$, it is convenient to split $\zeta$ according to (41) and define covariant derivatives $\nabla_{a}^{v}$ for $\zeta_{v}^{a} \in T_{\mathcal{Y}}^{v}$ and $\nabla_{i}^{h}$ for $\zeta_{h}^{i} \in T_{\mathcal{Y}}^{h}$ separately:

$$
\nabla_{b}^{v} \xi_{v}^{a}=\partial_{b} \xi_{v}^{a}+\gamma_{b c}^{a} \xi_{v}^{c}, \quad \nabla_{i}^{h} \xi_{v}^{a}=\partial_{i} \xi_{v}^{a}+\left(L_{V} \xi_{v}\right)_{i}^{a},
$$

where the Lie derivative $L_{V}$ along the vector field $V_{i}^{a}$ is defined as

$$
\left(L_{V} \xi_{v}\right)_{i}^{a}=V_{i}^{b} \partial_{b} \xi_{v}^{a}-\xi_{v}^{b} \partial_{b} V_{i}^{a} .
$$

Hence if $\xi_{v}$ is a vertical vector field, then its total covariant derivative is

$$
\nabla_{\beta} \xi_{v}^{a}=\partial_{\beta} \xi_{v}^{a}+\gamma_{b c}^{a} \nabla_{\beta} \phi^{b} \xi_{v}^{c}-\left(\partial_{b} V_{i}^{a}\right)\left(\partial_{\beta} \phi^{i}\right) \xi_{v}^{b} .
$$

Since both components (40) of $T_{\mathcal{Y}}$ have canonical affine connections, they define a canonical affine connection on $T_{\mathcal{Y}}$. It is given by

$$
\tilde{\nabla}_{B} \xi^{A}=\partial_{B} \xi^{A}+\tilde{\mathcal{A}}_{B C}^{A} \xi^{C}
$$

where

$$
\begin{gathered}
\tilde{\mathcal{A}}_{j k}^{i}=\hat{\Gamma}_{j k}^{i}, \quad \tilde{\mathcal{A}}_{b k}^{i}=\tilde{\mathcal{A}}_{j c}^{i}=\tilde{\mathcal{A}}_{b c}^{i}=0, \quad \tilde{\mathcal{A}}_{b c}^{a}=\gamma_{b c}^{a}, \\
\tilde{\mathcal{A}}_{k b}^{a}=\tilde{\mathcal{A}}_{b k}^{a}=-\gamma_{b c}^{a} V_{k}^{c}-\partial_{b} V_{k}^{a}, \\
\tilde{\mathcal{A}}_{j k}^{a}=-\partial_{j} V_{k}^{a}+V_{k}^{c} \partial_{c} V_{j}^{a}+\gamma_{b c}^{a} V_{j}^{b} V_{k}^{c}+V_{i}^{a} \hat{\Gamma}_{j k}^{i} .
\end{gathered}
$$

This connection has torsion:

$$
\mathcal{T}_{B C}^{A}=\tilde{\mathcal{A}}_{B C}^{A}-\tilde{\mathcal{A}}_{C B}^{A},
$$

whose only non-zero components are

$$
\mathcal{T}_{j k}^{a}=\mathcal{R}_{j k}^{a}
$$

Hence a modified connection

$$
\mathcal{A}_{B C}^{A}=\tilde{\mathcal{A}}_{B C}^{A}-\frac{1}{2} \mathcal{T}_{B C}^{A},
$$

is torsion-free. Its components are

$$
\begin{gathered}
\mathcal{A}_{j k}^{i}=\hat{\Gamma}_{j k}^{i}, \quad \mathcal{A}_{b k}^{i}=\mathcal{A}_{j c}^{i}=\mathcal{A}_{b c}^{i}=0, \quad \mathcal{A}_{b c}^{a}=\gamma_{b c}^{a}, \\
\mathcal{A}_{k b}^{a}=\mathcal{A}_{b k}^{a}=-\gamma_{b c}^{a} V_{k}^{c}-\partial_{b} V_{k}^{a}, \\
\mathcal{A}_{j k}^{a}=\frac{1}{2} \mathcal{R}_{j k}^{a}+\gamma_{b c}^{a} V_{j}^{b} V_{k}^{c}+V_{i}^{a} \hat{\Gamma}_{j k}^{i} .
\end{gathered}
$$


2.5.4. BRST transformations of boundary degrees of freedom. We consider the following fields on $\partial M$ :

$$
\text { bosonic: } \phi^{A}, \phi^{\bar{A}} \in \operatorname{Map}(\Sigma, \mathcal{Y}), F^{A} \in \Gamma\left(\phi^{*} T_{\mathcal{Y}} \otimes \Lambda^{2} T_{\Sigma}^{*}\right),
$$

$$
\text { fermionic: } H^{\bar{A}} \in \Gamma\left(\phi^{*} \bar{T}_{\mathcal{Y}}\right), P^{A} \in \Gamma\left(\phi^{*} T_{\mathcal{Y}} \otimes T_{\Sigma}^{*}\right), \theta_{a} \in \Gamma\left(\phi^{*} T_{\mathcal{Y}}^{v *}\right) .
$$

The fields are essentially those of the B-model with target $\mathcal{Y}$. Note though that instead of a fermionic 0 -form taking value in the pull-back of $T_{\mathcal{Y}}^{*}$, we have a field $\theta_{a}$ taking value in the pull-back of the quotient bundle $T_{\mathcal{Y}}^{*}=T_{\mathcal{Y}}^{*} / T_{\mathcal{Y}}^{* h}$. The bosonic field $F^{A}$ is an auxiliary field. The BRST transformations are taken to be

$$
\delta_{Q} \phi^{A}=0, \quad \delta_{Q} \phi^{\bar{A}}=H^{\bar{A}}, \quad \delta_{Q} H^{\bar{A}}=0, \quad \delta_{Q} P^{A}=d \phi^{A}, \quad \delta_{Q} \theta_{a}=0,
$$

and for the auxiliary field

$$
\delta_{Q} F^{A}=\nabla P^{A}+\frac{1}{2} \mathcal{R}_{B C \bar{D}}^{A} P^{B} P^{C} H^{\bar{D}}
$$

In the latter formula the covariant derivative and its curvature are those of (56-58): since the connection has zero torsion, the BRST transformation satisfies $\delta_{Q}^{2} F^{A}=0$. In fact, $\delta_{Q}$ is nilpotent when acting on any field, not just $F^{A}$.

Next we need to restrict the fields on the boundary so that for fixed values of the bulk fields they describe a B-model whose target is a fiber of $\mathcal{Y}$. We impose the following projection conditions:

$$
H^{\bar{i}}=\left.\eta^{\bar{i}}\right|_{\partial M}, \quad P^{i}=\left.\chi^{i}\right|_{\partial M}, \quad F^{i}=\operatorname{vol}_{\partial M} \Omega^{i j^{\prime}} A_{j^{\prime}}^{K} g_{K \bar{J}} \partial_{3} \phi^{\bar{J}} .
$$

The unrestricted parts of the fields can be parameterized by their projections to $T^{v} \mathcal{Y}$. Hence we introduce the fields

$$
\eta^{\bar{a}}=H^{\bar{a}}-V_{\bar{i}}^{\bar{a}} \eta^{\bar{i}}, \quad \rho^{a}=P^{a}-V_{i}^{a} \chi^{i}, \quad f^{a}=F^{a}-V_{i}^{a} F^{i} .
$$

Their BRST transformations can be computed to be

$$
\delta_{Q} \phi^{a}=0, \quad \delta_{Q} \phi^{\bar{a}}=\eta^{\bar{a}}+V_{\bar{i}}^{\bar{a}} \eta^{\bar{i}}, \quad \delta_{Q} \theta_{a}=0
$$

$$
\delta_{Q} \eta^{\bar{a}}=-\left(\partial_{\bar{b}} V_{\bar{i}}^{\bar{a}}\right) \eta^{\bar{b}} \eta^{\bar{i}}+\frac{1}{2} \mathcal{R}_{\bar{i} \bar{j}}^{\bar{a}} \eta^{\bar{i}} \eta^{\bar{j}}, \quad \delta_{Q} \rho^{a}=\nabla \phi^{a}-\mathcal{R}_{i \bar{j}}^{a} \eta^{\bar{j}} \chi^{i}-\left(\partial_{\bar{b}} V_{i}^{a}\right) \eta^{\bar{b}} \chi^{i}
$$




$$
\begin{aligned}
& \text { (67) } \delta_{Q} f^{a}=\nabla \rho^{a}+\mathcal{R}_{i \bar{j}}^{a} d \phi^{\bar{j}} \chi^{i}+\left(\partial_{\bar{b}} V_{i}^{a}\right) \nabla \phi^{\bar{b}} \chi^{i}+\frac{1}{2} \mathcal{R}_{b c \bar{d}}^{a} \rho^{b} \rho^{c} \eta^{\bar{d}} \\
& -\nabla_{c}\left(\partial_{\bar{d}} V_{j}^{a}\right) \chi^{j} \rho^{c} \eta^{\bar{d}}-\frac{1}{2} \nabla_{j}\left(\partial_{\bar{d}} V_{k}^{a}\right) \chi^{j} \chi^{k} \eta^{\bar{d}}+\left(-\nabla_{c} \mathcal{R}_{j \bar{l}}^{a}+\left(\partial_{c} V_{\bar{l}}^{\bar{b}}\right)\left(\partial_{\bar{b}} V_{j}^{a}\right)\right) \chi^{j} \rho^{c} \eta^{\bar{l}} \\
& +\frac{1}{2}\left(-\nabla_{j} \mathcal{R}_{k \bar{l}}^{a}+\left(\partial_{\bar{b}} V_{k}^{a}\right) \mathcal{R}_{\bar{l} j}^{\bar{b}}\right) \chi^{j} \chi^{k} \eta^{\bar{l}}-v o l_{\partial M}\left(\left(\partial_{\bar{b}} V_{i}^{a}\right) \eta^{\bar{b}}+R_{i \bar{j}}^{a} \eta^{\bar{j}}\right) \Omega^{i j^{\prime}} A_{j^{\prime}}^{K} g_{K \bar{J}} \partial_{3} \phi^{\bar{J}} .
\end{aligned}
$$

To compute the BRST transformation of $f^{a}$ we first used (62) for BRST transformations of $F^{a}$ and $F^{i}$ and then substituted the projected expressions (63). In (65-67) we used

$$
\nabla \phi^{a}=d \phi^{a}-V_{i}^{a} d \phi^{i}
$$

while the other covariant derivatives are defined as follows:

$$
\nabla \rho^{a}=d \rho^{a}+\gamma_{b c}^{a}\left(\nabla \phi^{b}\right) \rho^{c}-\left(\partial_{c} V_{j}^{a}\right)\left(d \phi^{j}\right) \rho^{c}+\frac{1}{2} \mathcal{R}_{k j}^{a}\left(d \phi^{k}\right) \chi^{j}
$$

$$
\nabla_{c}\left(\partial_{\bar{d}} V_{j}^{a}\right)=-\partial_{c} \partial_{\bar{d}} V_{j}^{a}+\gamma_{b c}^{a} \partial_{\bar{d}} V_{j}^{a},
$$

$$
\nabla_{j}\left(\partial_{\bar{d}} V_{k}^{a}\right)=\partial_{j} \partial_{\bar{d}} V_{k}^{a}-\hat{\Gamma}_{j k}^{i} \partial_{\bar{d}} V_{k}^{a}+\left(L_{V} \partial_{\bar{d}} V_{k}\right)_{j}^{a},
$$

$$
\left(L_{V} \partial_{\bar{d}} V_{k}\right)_{j}^{a}=V_{j}^{b} \partial_{b} \partial_{\bar{d}} V_{k}^{a}-\left(\partial_{\bar{d}} V_{k}^{b}\right) \partial_{b} V_{j}^{a},
$$$$
\text { (72) } \nabla_{c} \mathcal{R}_{j \bar{l}}^{a}=\partial_{c} \mathcal{R}_{j \bar{l}}^{a}+\gamma_{b c}^{a} \mathcal{R}_{j \bar{l}}^{b} \text {, }
$$$$
\text { (73) } \nabla_{j} \mathcal{R}_{k \bar{l}}^{a}=\partial_{j} \mathcal{R}_{k \bar{l}}^{a}-\hat{\Gamma}_{j k}^{i} \mathcal{R}_{i \bar{l}}^{a}+\left(L_{V} \mathcal{R}_{k \bar{l}}\right)_{j}^{a} \text {, }
$$

$$
\left(L_{V} \mathcal{R}_{k \bar{l}}\right)_{j}^{a}=V_{j}^{b} \partial_{b} \mathcal{R}_{k \bar{l}}^{a}-\mathcal{R}_{k \bar{l}}^{b} \partial_{b} V_{j}^{a} .
$$

Note that if the Ehresmann connection is zero, $V_{i}^{a}=0$, then the equations (65-67) become BRST transformations of the B-model with target space $Z$.

2.5.5. The boundary action. Now we can construct a covariant and BRST-invariant boundary action $S^{b r y}=\int_{\partial M} \mathcal{L}^{b r y}$ which for trivial Ehresmann connection becomes the usual action of B-model:

$$
\begin{gathered}
\mathcal{L}^{b r y}=\mathcal{L}_{1}^{b r y}+\mathcal{L}_{2}^{b r y}, \\
\mathcal{L}_{1}^{b r y}=\delta_{Q}\left(\mathcal{V}_{1}^{b r y}\right), \quad \mathcal{V}_{1}^{b r y}=g_{a \bar{b}} \rho^{a} \wedge * \nabla \phi^{\bar{b}},
\end{gathered}
$$




$$
\begin{gathered}
\mathcal{L}_{1}^{b r y}=\operatorname{vol}_{\partial M} h^{\alpha \beta}\left[g_{a \bar{b}}\left(\nabla_{\alpha} \phi^{a}\right)\left(\nabla_{\beta} \phi^{\bar{b}}\right)-g_{a \bar{b}} \rho_{\alpha}^{a} \nabla_{\beta} \eta^{\bar{b}}+\left(\nabla_{\bar{i}} g_{a \bar{b}}\right)\left(\nabla_{\alpha} \phi^{\bar{b}}\right) \eta^{\bar{i}} \rho_{\beta}^{a}\right. \\
+\left(\mathcal{R}_{\bar{i} j}^{\bar{b}} \partial_{\alpha} \phi^{j}+\mathcal{R}_{\bar{i} \bar{b}}^{\bar{b}} \partial_{\alpha} \phi^{\bar{j}}+\left(\partial_{c} V_{\bar{i}}^{\bar{b}}\right) \nabla_{\alpha} \phi^{c}\right) g_{a \bar{b}} \eta^{\bar{i}} \rho_{\beta}^{a}-g_{a \bar{b}} \mathcal{R}_{i \bar{j}}^{a}\left(\nabla_{\alpha} \phi^{\bar{b}}\right) \eta^{\bar{j}} \chi_{\beta}^{i} \\
\left.-g_{a \bar{b}}\left(\partial_{\bar{c}} V_{i}^{a}\right)\left(\nabla_{\alpha} \phi^{\bar{b}}\right) \eta^{\bar{c}} \chi_{\beta}^{i}\right] \\
\mathcal{L}_{2}^{b r y}=-\theta_{a} \delta_{Q} f^{a} .
\end{gathered}
$$

BRST variation of the boundary action (75-78) is given by

$$
\delta_{Q} \mathcal{L}^{b r y}=\Omega^{i j^{\prime}}\left(\left(\partial_{\bar{b}} V_{i}^{a}\right) \eta^{\bar{b}}+\mathcal{R}_{i \bar{j}}^{a} \eta^{\bar{j}}\right) \theta_{a} A_{j^{\prime}}^{K} \frac{\delta S^{b u l k}}{\delta \chi_{3}^{K}} .
$$

Let us modify BRST transformation of the bulk field $\chi_{3}^{K}$ in the following way:

$$
\tilde{\delta}_{Q} \chi_{3}^{K}=\partial_{3} \phi^{K}-\delta\left(x_{3}\right) A_{j^{\prime}}^{K} \Omega^{j^{\prime} i} \theta_{a}\left(\left(\partial_{\bar{b}} V_{i}^{a}\right) \eta^{\bar{b}}+\mathcal{R}_{i \bar{j}}^{a} \eta^{\bar{j}}\right) .
$$

Then the total action $S^{\text {tot }}=\int_{M} \mathcal{L}^{b u l k}+\int_{\partial M} \mathcal{L}^{b r y}$ is BRST invariant, and moreover $\mathcal{L}_{1}^{\text {bulk }}$ combines with the last term in $\mathcal{L}_{2}^{\text {bry }}$ into

$$
\tilde{\delta}_{Q}\left(\mathcal{V}_{1}^{b u l k}\right)
$$

so that the dependence of $S^{t o t}$ on the metric on $M$ enters only through BRST-exact terms.

The boundary term in the BRST variation of $\chi_{3}^{K}$ is proportional to

$$
\left(\partial_{\bar{b}} V_{i}^{a}\right) \eta^{\bar{b}}+\mathcal{R}_{i \bar{j}}^{a} \eta^{\bar{j}}=\partial_{\bar{A}} V_{i}^{a} H^{\bar{A}}
$$

The tensor

$$
\mathcal{F}_{i \bar{A}}^{a}=\partial_{\bar{A}} V_{i}^{a}
$$

is a $\bar{\partial}$-closed section of $\Omega^{0,1}\left(T_{\mathcal{Y}}^{v} \otimes T_{\mathcal{Y}}^{h *}\right)$, and its cohomology class has a simple geometric meaning: it is the Atiyah class of the fibration $\mathcal{Y} \rightarrow$ $Y$, i.e. an obstruction to the existence of a holomorphic Ehresmann connection.

The Atiyah class also corrects the boundary BRST variations of other fields. Let us consider the field $\phi^{I}$. The boundary action (78) contains a term proportional to $\partial_{3} \phi^{\bar{J}}$, therefore $\partial_{3} \phi^{I}$ has a delta-function singularity on the boundary:

$$
\partial_{3} \phi^{I}=\delta\left(x^{3}\right) A_{k^{\prime}}^{I} \Omega^{k^{\prime} j} \theta_{a} H^{\bar{A}} \mathcal{F}_{j \bar{A}}^{a} .
$$


This means that $\phi^{I}$ itself has a step-like discontinuity on the boundary. Using (28) we find

$$
\tilde{\delta}_{Q} \phi^{i}=\theta\left(-x^{3}\right) \beta_{\bar{k}}^{i j} \mathcal{F}_{j \bar{A}}^{a} \theta_{a} \eta^{\bar{k}} H^{\bar{A}} .
$$

This should be regarded as the leading quantum correction to the BRST variation of $\phi^{i}$.

Once the BRST transformations for $\phi^{i}$ are modified, covariance with respect to holomorphic changes of coordinates on $\mathcal{Y}$ requires one to modify BRST transformations for $\phi^{a}$. In mathematical terms, one needs to lift the cohomology class $[\beta \mathcal{F}] \in H^{2}\left(T_{\mathcal{Y}}^{h} \otimes T_{\mathcal{Y}}^{v}\right)$ to $H^{2}\left(T_{\mathcal{Y}} \otimes T_{\mathcal{Y}}^{v}\right)$. That is, we would like to find a $\bar{\partial}$-closed section of $T_{\mathcal{Y}} \otimes T_{\mathcal{Y}}^{v} \otimes \Omega_{\mathcal{Y}}^{0,2}$ whose image under the projection $T_{\mathcal{Y}} \rightarrow T_{\mathcal{Y}}^{h}$ is the section

$$
\beta_{\bar{k}}^{i j} \mathcal{F}_{j \bar{A}}^{a} \partial_{i} \otimes \partial_{a} \otimes d \phi^{\bar{k}} \wedge d \phi^{\bar{A}} \in \Omega^{0,2}\left(T_{\mathcal{Y}}^{h} \otimes T_{\mathcal{Y}}^{v}\right) .
$$

From the short exact sequence

$$
0 \rightarrow T_{\mathcal{Y}}^{v} \rightarrow T_{\mathcal{Y}} \rightarrow T_{\mathcal{Y}}^{h} \rightarrow 0
$$

we see that there is an obstruction for doing this taking values in $H^{3}\left(T_{\mathcal{Y}}^{v} \otimes T_{\mathcal{Y}}^{v}\right)$

To write the obstruction and the modified transformation law for $\phi^{a}$ more explicitly, we will adopt the following notation. We will regard inhomogeneous forms of type $(0, p)$ as functions on the odd tangent bundle of $\mathcal{Y}$, i.e. as functions of odd fields $H^{\bar{A}}$, and will keep this dependence implicit. Thus $\beta^{i j}$ will mean $\beta_{\bar{k}}^{i j} \eta^{\bar{k}}$, and $\mathcal{F}_{j}^{a}$ will mean $\mathcal{F}_{j \bar{A}}^{a} H^{\bar{A}}$. Then the most general covariant transformation law for $\phi^{a}$ can be written as

$$
\tilde{\delta}_{Q} \phi^{a}=V_{i}^{a} \beta^{i j} \mathcal{F}_{j}^{b} \theta_{b}+\rho^{a},
$$

where $\rho^{a}$ is a section of $T_{\mathcal{Y}}^{v}$ depending on other fields. Requiring $\tilde{\delta}_{Q}^{2} \phi^{a}=$ 0 we find that $\rho^{a}$ has the form

$$
\rho^{a}=\rho^{a b} \theta_{b},
$$

where $\rho^{a b}$ satisfies

$$
\bar{\partial} \rho^{a b}=\beta^{i j} \mathcal{F}_{i}^{a} \mathcal{F}_{j}^{b}
$$

This means that the cohomology class of

$$
\beta^{i j} \mathcal{F}_{i}^{a} \mathcal{F}_{j}^{b} \in \Omega^{0,3}\left(\Lambda^{2} T_{\mathcal{Y}}^{v}\right),
$$

must be trivial. This cohomology class is an obstruction for coupling the B-model with target $\mathcal{Y}$ and the RW model with target $X$.

If the obstruction vanishes, then one picks a particular solution $\rho^{a b}$ of (82) and defines the modified BRST transformation laws for $\phi^{a}$ by

$$
\tilde{\delta}_{Q} \phi^{a}=\left(\rho^{a b}+V_{i}^{a} \beta^{i j} \mathcal{F}_{j}^{b}\right) \theta_{b} .
$$


One may always choose $\rho^{a b}$ to be antisymmetric.

BRST transformations of the fields $\theta_{a}$ and $P^{A}$ also need to be corrected to ensure that the boundary action is BRST-invariant. In effect, this amounts to a further deformation of the boundary B-model. We will not pursue this issue here.

2.5.6. Boundary local observables. Local observables on the boundary are constructed in complete analogy with the B-model:

$$
\mathcal{O}_{\omega}=\omega_{\bar{A}_{1} \ldots \bar{A}^{s}}^{a_{1} \ldots a_{r}} H^{\bar{A}_{1}} \ldots H^{\bar{A}_{s}} \theta_{a_{1}} \ldots \theta_{a_{r}}, \quad \omega \in \Omega^{0, s}\left(\Lambda^{r} T_{\mathcal{Y}}^{v}\right) .
$$

On the classical level, the BRST operator acts by

$$
\delta_{Q} \mathcal{O}_{\omega}=\mathcal{O}_{\bar{\partial} \omega} .
$$

Thus we may identify the classical algebra of boundary topological observables with

$$
\oplus_{s, r} H^{s}\left(\Lambda^{r} T_{\mathcal{Y}}^{v}\right)
$$

As discussed above, the BRST differential receives quantum corrections. Eqs. (81) and (84) tell us how the BRST variation of $\phi^{A}$ is modified to leading order in the Planck constant. Since we have not computed how the BRST variation of $\theta_{a}$ is modified, we will only consider the observables which do not depend on $\theta_{a}$. More precisely, we expand an observable $W$ into a Taylor series in the Planck constant, $W=W_{0}+W_{1}+\ldots$, where $W_{k}$ is of order $\hbar^{k}$, and require $W_{0}$ to depend only on $\phi^{A}, \phi^{\bar{A}}$ and $H^{\bar{A}}$. Then BRST-invariance requires $W_{0}$ to satisfy

$$
\bar{\partial} W_{0}=0,
$$

while $W_{1}$ satisfies

$$
\bar{\partial} W_{1}+\beta^{i j} \mathcal{F}_{j}^{b} \theta_{b} \nabla_{i} W_{0}+\rho^{a b} \partial_{a} W_{0} \theta_{b}=0 .
$$

From this equation we see that $W_{1}$ must have the form $W_{1}=\psi^{a} \theta_{a}$, where $\psi^{a}$ is an element of $\Omega^{0, \hat{1}}\left(T_{\mathcal{Y}}^{v}\right)$ satisfying

$$
\bar{\partial} \psi^{a}=-\beta^{i j} \mathcal{F}_{j}^{a} \nabla_{i} W_{0}+\rho^{a b} \partial_{b} W_{0} .
$$

It follows from (82) that the right-hand-side of (86) is $\bar{\partial}$-closed and therefore defines a class in $H^{\hat{0}}\left(T_{\mathcal{Y}}^{v}\right)$. For the equation (86) to admit solutions, this cohomology class must be trivial. One can interpret this mathematically by saying that there is a spectral sequence which converges to the quantum algebra of boundary observables whose first term is given by (85) and the action of the first differential on the subspace with $r=1$ is given by the right-hand-side of (86). 
2.5.7. Deformations of the boundary action. Infinitesimal deformations of the boundary action correspond to even elements of the algebra of boundary observables. Let us again consider an observable $W=$ $W_{0}+W_{1}+\ldots$ such that $W_{0}$ does not depend on $\theta_{a}$. On the classical level, we may drop all the terms except $W_{0}$, and the BRST invariance requires $W_{0}$ to satisfy $\bar{\partial} W_{0}=0$. The corresponding deformation of the boundary action is obtained by applying the descent procedure. The results are very much like in subsection 2.4, with $Y$ replaced with the total space of $\mathcal{Y}$ :

$$
W^{(1)}=P^{A} \partial_{A} W+d \phi^{\bar{A}} \frac{\partial W}{\partial H^{\bar{A}}},
$$

$$
\begin{aligned}
W^{(2)} & =\frac{1}{2} P^{A} P^{B} \nabla_{A} \partial_{B} W+P^{A} d \phi^{\bar{B}} \partial_{A} \frac{\partial W}{\partial H^{\bar{B}}}+\frac{1}{2} d \phi^{\bar{A}} d \phi^{\bar{B}} \frac{\partial^{2} W}{\partial H^{\bar{A}} \partial H^{\bar{B}}} \\
& -\operatorname{vol}_{\partial M} \Omega^{k^{\prime} i}\left(\partial_{i} W+V_{i}^{a} \partial_{a} W\right) A_{k^{\prime}}^{K} g_{K \bar{J}} \partial_{3} \phi^{\bar{J}} .
\end{aligned}
$$

The descendants satisfy

$$
\delta_{Q} W^{(1)}=d W, \quad \delta_{Q} W^{(2)}=d W^{(1)}+\partial_{a} W \frac{\delta S^{b r y}}{\delta \theta_{a}}+\Omega^{k^{\prime} i}\left(\partial_{i} W+V_{i}^{a} \partial_{a} W\right) A_{k^{\prime}}^{K} \frac{\delta S^{b u l k}}{\delta \chi_{3}^{K}} .
$$

We define the deformed action to be

$$
S^{\text {tot def }}=\int_{M} \mathcal{L}^{\text {bulk }}+\int_{\partial M} \mathcal{L}^{b r y}+\int_{\partial M} W^{(2)} .
$$

and modify the BRST transformation for the fields $\chi_{3}^{K}$ and $\theta_{a}$ :

$$
\tilde{\delta}_{Q, W} \chi_{3}^{K}=\partial_{3} \phi^{K}-\delta\left(x_{3}\right) A_{k^{\prime}}^{K} \Omega^{k^{\prime} i} \theta_{a} H^{\bar{A}} \mathcal{F}_{i \bar{A}}^{a}-\delta\left(x^{3}\right) A_{k^{\prime}}^{K} \Omega^{k^{\prime} i}\left(\partial_{i} W+V_{i}^{a} \partial_{a} W\right),
$$

$$
\tilde{\delta}_{Q, W} \theta_{a}=-\partial_{a} W \text {. }
$$

Then the total action is BRST-invariant up to a total derivative:

$$
\tilde{\delta}_{Q, W} S^{\text {tot def }}=\int_{\partial M} d W^{(1)}
$$

In particular, if we take $W$ to be a holomorphic function on $\mathcal{Y}$, the deformed action describes a family of Landau-Ginzburg models fibered over $Y$, with the superpotential depending both on the fiber and base coordinates. In general, we will call $W$ the curving, since in section $\mathrm{B}$ the B-model deformed by an even inhomogeneous $\bar{\partial}$-closed form is called the curved B-model. 
As discussed above, for a given $W_{0}$ there are obstructions for finding $W_{1}, W_{2}, \ldots$, satisfying the requirements of BRST invariance. However even if all these obstructions vanish, there may be an obstruction to extending an infinitesimal deformation $W=W_{0}+W_{1}+\ldots$ to a finite deformation already at second order in $W$. This is a quantum effect which arises because adding $W^{(2)}$ to the action necessitates a change in the BRST transformations of fields on the boundary. As in section 2.4.1, we can determine the modification of the BRST transformation of $\phi^{i}$ by noting that $\phi^{I}$ has a discontinuity at $x^{3}=0$ :

$$
\partial_{3} \phi^{K}=\delta\left(x^{3}\right) A_{k^{\prime}}^{K} \Omega^{k^{\prime} j}\left(-\mathcal{F}_{j}^{a} \theta_{a}+\nabla_{j} W_{0}\right)
$$

Then the BRST variation of $\phi^{i}$ is given by

$$
\tilde{\delta}_{Q, W} \phi^{i}=\theta\left(-x^{3}\right) \beta^{i j}\left(\mathcal{F}_{j}^{a} \theta_{a}-\nabla_{j} W_{0}\right) .
$$

(We replaced $W$ with $W_{0}$ because we are only interested in leading quantum corrections to BRST transformations). It is easy to see that this ensures $\tilde{\delta}_{Q, W}^{2} \chi_{3}^{K}=0$. One can also check that $\tilde{\delta}_{Q, W}^{2} \phi^{i}=0$.

Once the BRST transformation of $\phi^{i}$ has been modified, covariance with respect to changes of coordinates requires us to modify the BRST transformation of $\phi^{a}$. The most general covariant BRST variation is

$$
\tilde{\delta}_{Q, W} \phi^{a}=V_{i}^{a} \tilde{\delta}_{Q, W} \phi^{i}+\rho_{W}^{a},
$$

where $\rho_{W}^{a}$ is a field-dependent and possibly $W$-dependent section of $T_{\mathcal{Y}}^{v}$. Requiring $\tilde{\delta}_{Q, W}^{2} \phi^{a}=0$, we find that $\rho_{W}^{a}$ must have the form

$$
\rho_{W}^{a}=\rho_{W}^{a b} \theta_{b}+\psi_{W}^{a},
$$

where $\rho_{W}^{a b}$ and $\psi_{W}^{a}$ are independent of $\theta_{a}$ and satisfy

$$
\begin{aligned}
& \bar{\partial} \rho_{W}^{a b}=\beta^{i j} \mathcal{F}_{i}^{a} \mathcal{F}_{j}^{b}, \\
& \bar{\partial} \psi_{W}^{a}=\rho^{a b} \partial_{b} W_{0}-\beta^{i j} \mathcal{F}_{i}^{a} \nabla_{j} W_{0} .
\end{aligned}
$$

Comparing these equations with (82) and (86) we see that particular solutions are obtained by letting

$$
\rho_{W}^{a b}=\rho^{a b}, \quad \psi_{W}^{a}=\psi^{a} .
$$

We now found the leading quantum corrections to BRST transformations of $\phi^{A}$ including terms linear in $W_{0}$. The obstruction quadratic in $W_{0}$ arise from the requirement that $W$ be BRST-invariant with respect to these corrected transformations. This requirement implies that $W_{1}$ must have the form

$$
W_{1}=\psi^{a} \theta_{a}+w
$$

where $w$ is independent of $\theta_{a}$ and satisfies

$$
\bar{\partial} w=-2 \psi^{a} \partial_{a} W_{0}+\beta^{i j} \nabla_{i} W_{0} \nabla_{j} W_{0} .
$$


The right-hand-side of this equation is $\bar{\partial}$-closed, thanks to (86) and the antisymmetry of $\rho^{a b}$. Thus it defines a cohomology class in $H^{\hat{1}}\left(\mathcal{O}_{\mathcal{Y}}\right)$ which is an obstruction for the existence of $w$. This is the first obstruction for extending an infinitesimal deformation $W$ to a finite deformation; as expected, it is quadratic in $W$.

2.6. Reduction on a circle in the presence of boundaries. Let $M=S^{1} \times \Sigma$, where the Riemann surface $\Sigma$ has a nonempty boundary. Since the dimensional reduction of the RW model is the B-model with the same target, every boundary condition in the RW model gives rise to a boundary condition for the B-model. In this subsection we will determine which B-branes can be obtained in this way.

First let us consider the case without boundary degrees of freedom, i.e. when $\mathcal{Y}=Y$. Let $S^{1}$ be parameterized by $x^{2} \in[0,2 \pi)$. The reduction to a $2 \mathrm{~d}$ field theory occurs in the limit when the circumference of $S^{1}$ goes to zero, i.e. $h_{22} \rightarrow 0$. We decompose the 1 -form $\chi$ into components along $\Sigma$ and $S^{1}$ :

$$
\chi^{I}=\chi_{\Sigma}^{I}+\chi_{2}^{I} d x^{2}
$$

Reduction along $S^{1}$ amounts to requiring all fields to be independent of $x^{2}$, so $\chi_{\Sigma}^{I}$ is a pull-back of a 1 -form $\rho^{I}$ on $\Sigma$. We also define

$$
\theta_{I}=\int \Omega_{I J} \chi_{2}^{J} d x^{2}
$$

The reduced model also contains fields $\eta^{\bar{I}}, \phi^{I}$ and $\phi^{\bar{I}}$. Their BRST transformations are

$$
\delta \phi^{I}=0, \quad \delta \phi^{\bar{I}}=\eta^{\bar{I}}, \quad d \rho^{I}=d \phi^{I}, \quad \delta \theta_{I}=0 .
$$

These are the BRST transformations of the B-model. On the boundary $\partial \Sigma$ the bosonic fields $\phi^{I}, \phi^{\bar{I}}$ take values in $Y \subset X$, while the conjugate momenta satisfy

$$
\pi_{\phi^{i}}=\frac{\partial \mathcal{L}^{\text {bulk }}}{\partial\left(\partial_{3} \phi^{i}\right)}=0, \quad \pi_{\phi^{\bar{i}}}=\frac{\partial \mathcal{L}^{\text {bulk }}}{\partial\left(\partial_{3} \phi^{\bar{i}}\right)}=0 .
$$

The fermionic fields satisfy the boundary conditions

$$
\eta^{\bar{i}^{\prime}}=0, \quad \theta_{i}=0, \quad g_{\bar{i} J} \rho_{3}^{J}=0, \quad \rho_{1}^{i^{\prime}}=0 .
$$

These are precisely the boundary conditions for a B-brane corresponding to the submanifold $Y \subset X$.

In general, when boundary degrees of freedom are present, we have to perform the reduction both for bulk and boundary degrees of freedom. For simplicity, let us consider the case when the fiber of $\mathcal{Y}$ is compact. If the bulk fields are fixed, the space of states of boundary degrees of 
freedom is the space of states of the curved B-model whose target is the fiber of $\mathcal{Y}$. If the fiber is Kähler, this space is the de Rham cohomology of the fiber, with the natural $\mathbb{Z}_{2}$ grading (see section $\mathrm{B}$ ). As one varies the boundary values of the bulk fields, these $\mathbb{Z}_{2}$-graded vector spaces fit into a vector bundle which can be described as fiberwise de Rham cohomology. This vector bundle is flat, the flat connection being the Gauss-Manin connection. We conclude that a Lagrangian submanifold $Y$ equipped with a fibration $\mathcal{Y}$ whose fibers are compact and Kähler reduces to a B-brane $Y$ equipped with a flat vector bundle (the fiberwise de Rham cohomology of $\mathcal{Y})$.

2.7. Reduction on an interval. We have explained above that the RW theory with target $X$ on $S^{1} \times \Sigma$ is equivalent to the B-model with the same target on $\Sigma$. We can also consider the RW model on a manifold of the form $I \times \Sigma$, where $I$ is an interval. The boundary of this 3-manifold is a disjoint union of two copies of $\Sigma$, so we can choose two different boundary conditions corresponding to two complex Lagrangian submanifolds $Y_{1}, Y_{2}$ equipped with fibrations $\mathcal{Y}_{1}, \mathcal{Y}_{2}$ over them. In this situation the RW theory must reduce to some $2 \mathrm{~d}$ topological field theory on $\Sigma$. In this subsection we will determine this theory. This will enable us in the next section to identify the categories of line defects separating different boundary conditions.

2.7.1. Identical submanifolds at the two ends. Since the general case is rather complicated, we will first consider some special cases. The simplest case is $Y_{1}=Y_{2}=Y$, and the boundary degrees of freedom are absent: $\mathcal{Y}_{1}=\mathcal{Y}_{2}=Y$. The boundary conditions restrict the bosonic fields to the submanifold $Y$. If we expand all bosonic fields into Fourier series on the interval $I$, then all the modes for $\phi^{i^{\prime}}$ will be massive, while the constant mode for $\phi^{i}$ will be massless. The masses of the massive modes are of order $1 / \sqrt{h_{33}}$, so in the limit $h_{33} \rightarrow 0$ one can set $\phi^{i^{\prime}}=0$ and assume that the fields $\phi^{i}$ are constant on $I$. Similarly, the massless fermionic fields are constant modes of $\eta^{\bar{i}}, \chi_{1,2}^{i}$, and

$$
\theta_{i}=\int \Omega_{i j^{\prime}} \chi_{3}^{j^{\prime}} d x^{3}
$$

These are the fields of the B-model with target $Y$. It is easy to check that the BRST transformations and the action are also those of the B-model with target $Y$.

The above derivation of the effective $2 \mathrm{~d}$ theory was classical; now let us analyze possible quantum corrections. As in the case of the circle reduction, on the quantum level it is not correct simply to set nonconstant modes to zero: one must integrate them out, and this could 
induce corrections to the effective $2 \mathrm{~d}$ theory. We will now show that ghost number symmetry does not allow any such corrections. Quantum corrections would deform the B-model while preserving the ghost number symmetry and therefore would take values in

$$
\oplus_{p+q=2} H^{p}\left(\Lambda^{q} T_{Y}\right) .
$$

The corresponding class must be built from the curvature tensor of $Y$, which represents a class in $H^{1}\left(\Omega^{1} \otimes \operatorname{End} T_{Y}\right)$, and the classes $\beta^{(\ell)} \in$ $H^{1}\left(\operatorname{Sym}^{\ell} T_{Y}\right), \ell \geq 2$, describing the formal neighborhood of $Y$. Consider a monomial which contains $m$ curvature tensors and the classes $\beta^{\left(\ell_{i}\right)}, i=1, \ldots, N$. The integers $p$ and $q$ in (95) are given by

$$
p=m+N, \quad q=\sum_{i} \ell_{i}-m .
$$

Since $p+q=2$, we must have

$$
\sum_{i} \ell_{i}+N=2
$$

But since $\ell_{i} \geq 2 \forall i$ and $N>0$, this equality cannot be satisfied.

The quantum B-model with target $Y$ is anomalous unless $Y$ is a halfCalabi-Yau manifold, i.e. unless the square of the canonical line bundle $K_{Y}$ is trivial. Since a consistent $3 \mathrm{~d}$ topological field field theory must reduce to a consistent $2 \mathrm{~d}$ topological field theory, we conclude that the RW model on a 3-manifold with boundary is anomalous unless $Y$ is a half-Calabi-Yau manifold. In fact, it is easy to see that the RW model is anomalous unless $Y$ is a Calabi-Yau manifold. Consider the path-integral on a ball $D^{3}$ on whose boundary we specify a boundary condition associated to $Y$. The bosonic zero modes are $\phi^{i}, \phi^{\bar{i}}, i=$ $1, \ldots, n$, the fermionic zero modes are $\eta^{\bar{i}}, i=1, \ldots, n$, and a BRSTinvariant measure

$$
\omega_{i_{1} \ldots i_{n}} d \phi^{i_{1}} \ldots d \phi^{i_{n}} d \phi^{\overline{1}} \ldots d \phi^{\bar{n}} d \eta^{\overline{1}} \ldots d \eta^{\bar{n}}
$$

requires a choice of a holomorphic volume form $\omega_{i_{1} \ldots i_{n}}$ on $Y$. Thus $K_{Y}$ must be trivial.

2.7.2. Including the curving. Now let us keep $\mathcal{Y}_{1}=\mathcal{Y}_{2}=Y_{1}=Y_{2}=$ $Y$, but allow for nontrivial curvings $W_{1}$ and $W_{2}$ on $Y_{1}$ and $Y_{2}$. An important difference compared to the previous case is that the field $\phi^{K}$ has step-like discontinuities at $x^{3}=0$ and $x^{3}=1$ :

$$
\begin{aligned}
& \lim _{x^{3} \rightarrow 0+} \phi^{K}\left(x^{1}, x^{2}, x^{3}\right)-\phi^{K}\left(x^{1}, x^{2}, 0\right)=A_{k^{\prime}}^{K} \Omega^{k^{\prime} i} \partial_{i} W_{1}, \\
& \lim _{x^{3} \rightarrow 1-} \phi^{K}\left(x^{1}, x^{2}, x^{3}\right)-\phi^{K}\left(x^{1}, x^{2}, 0\right)=A_{k^{\prime}}^{K} \Omega^{k^{\prime} i} \partial_{i} W_{2} .
\end{aligned}
$$


As a consequence, the field $\phi^{I}$ on the interval $(0,1)$ will not be constant either, even in the limit when its length goes to zero. To determine the reduced model, a convenient short-cut is to focus on BRST transformations of the fields. The action of the B-model will be determined by these BRST transformations uniquely, up to BRST-exact terms.

The bosonic fields of the reduced model are

$$
\phi_{0}^{i}=\int_{0}^{1} \phi^{i} d x^{3}, \quad \phi_{0}^{\bar{i}}=\int_{0}^{1} \phi^{\bar{i}} d x^{3} .
$$

The fermionic 0-form fields are

$$
\eta_{0}^{\bar{i}}=\int_{0}^{1} \eta^{\bar{i}} d x^{3}, \quad \theta_{i}=\int_{0}^{1} \Omega_{i k^{\prime}} \chi_{3}^{k^{\prime}} d x^{3} .
$$

The fermionic 1-form fields are

$$
\rho^{i}=\int_{0}^{1} \chi_{\Sigma}^{i} d x^{3}
$$

The BRST variations of the fields are

$$
\begin{aligned}
\delta_{Q, W} \phi_{0}^{i} & =0, & & \delta_{Q, W} \phi_{0}^{\bar{i}}=\eta_{0}^{\bar{i}}, \\
\delta_{Q, W} \eta_{0}^{\bar{i}} & =0, & \delta_{Q, W} \theta_{i} & =-\partial_{i}\left(W_{1}-W_{2}\right), \\
\delta_{Q, W} \rho^{i} & =d \phi_{0}^{i}, & &
\end{aligned}
$$

where $W_{1,2}$ are regarded as functions of $\phi_{0}^{i}, \phi_{0}^{\bar{i}}$, and $\eta_{0}^{\bar{i}}$.

The field content and BRST transformations of the reduced model appear to be the same as in the curved B-model with target $Y$ and curving $W_{1}-W_{2}$. But on the quantum level there are corrections to this statement which originate from the fact that in general the formal neighborhood of $Y$ differs from $T_{Y}^{*}$. Indeed, the fields $\phi^{i^{\prime}}$ have discontinuities at $x^{3}=0,1$, and since the transition functions for $\phi^{i}$ depend on the values of $\phi^{i^{\prime}}$, this affects the transition functions for $\phi_{0}^{i}$, i.e. the complex structure of the target of the B-model. To leading order in $\phi^{i^{\prime}}$, this modification of the complex structure of $Y$ is represented by a Cech 1-cocycle with values in $T_{Y}$ which represents the cohomology class $[\beta(\xi)]$, where $\beta \in H^{1}\left(T_{Y} \otimes N_{Y}^{*}\right)$ is defined by the exact sequence (23), and $\xi$ is the normal vector field on $Y$ specifying the discontinuity of $\phi^{i^{\prime}}$.

In our case $\xi$ is given by

$$
\xi^{k^{\prime}}=\Omega^{k^{\prime} i} \partial_{i} W
$$

where $W$ is either $W_{1}$ or $W_{2}$ depending on whether we take $x^{3}=0$ or $x^{3}=1$. In the limit $h_{33} \rightarrow 0$ we may assume that $\phi^{i^{\prime}}$ depends linearly on $x^{3}$, so the deformation of the transition function for $\phi_{0}^{i}$ is 
the average of the deformations at $x^{3}=0$ and $x^{3}=1 .^{5}$ The conclusion is that the reduced theory is a B-model whose target is a deformation of $Y$ corresponding to the cohomology class

$$
\left[\frac{1}{2} \beta\left(\partial\left(W_{1}+W_{2}\right)\right]\right.
$$

Note that since $W_{1,2}$ are not simply functions, but $\bar{\partial}$-closed $(0, \hat{0})$ forms, $\xi$ is not really a normal vector field but a $\bar{\partial}$-closed section of $\Omega^{0,0}\left(N_{Y}\right)$. Consequently, the class above takes values not in $H^{1}\left(T_{Y}\right)$, but in $H^{\hat{1}}\left(T_{Y}\right)$, and the modified "transition functions" are not holomorphic functions but invertible $\bar{\partial}$-closed inhomogeneous forms of type $(0, p)$ with even $p$. If desired, one can make a change of field variables $\phi_{0}^{i}, \phi_{0}^{\bar{i}}$ and $\eta_{0}^{\bar{i}}$ which eliminates such strange "transition functions", but at the expense of modifying the BRST transformations for $\phi_{0}^{i}$. If $\beta$ is a Dolbeault representative of $[\beta]$, the corresponding modification of the BRST transformation for $\phi_{0}$ will read

$$
\delta \phi_{0}^{i}=-\frac{1}{2} \beta^{i j} \partial_{j}\left(W_{1}+W_{2}\right)
$$

Since the curvings $W_{1}, W_{2}$ depend on the fields $\eta_{0}^{\bar{j}}$, the right-hand-side of this formula may contain arbitrary odd powers of $\eta_{0}^{\bar{j}}$.

2.7.3. Including the fibrations. The next case is $Y_{1}=Y_{2}=Y$, but the fibrations $\mathcal{Y}_{1}, \mathcal{Y}_{2}$ are otherwise arbitrary. We also allow for curvings $W_{1}$ and $W_{2}$ on $\mathcal{Y}_{1}$ and $\mathcal{Y}_{2}$. For simplicity we will neglect the effects of the class $\beta$, i.e. the discussion will be classical. As before, in the $2 \mathrm{~d}$ limit the bosonic fields $\phi^{i^{\prime}}$ vanish, while the boundary degrees of freedom at $x^{3}=0$ and $x^{3}=1$ are not affected by the reduction. Thus the reduced theory will be a sigma-model with target $\mathcal{Y}_{1} \times_{Y} \mathcal{Y}_{2}$, where $\times_{Y}$ denotes the fibered product. The map from $\Sigma$ to $\mathcal{Y}_{1} \times_{Y} \mathcal{Y}_{2}$ can be described by the bosonic fields $\phi^{A}=\left(\phi^{i}, \phi_{1}^{a_{1}}, \phi_{2}^{a_{2}}\right)$.

Let $\pi_{1}$ and $\pi_{2}$ be the projections from $\mathcal{Y}_{1} \times_{Y} \mathcal{Y}_{2}$ to $\mathcal{Y}_{1}$ and $\mathcal{Y}_{2}$, respectively, and $p_{12}$ be the projection from $\mathcal{Y}_{1} \times_{Y} \mathcal{Y}_{2}$ to $Y$. The fermionic fields of the reduced model come from the boundary fermionic fields and the constant Fourier modes of the bulk fields. The former give rise

\footnotetext{
${ }^{5}$ For $W_{1} \neq W_{2}$, we cannot assume that $\phi^{i^{\prime}}$ is independent of $x^{3}$ because such an ansatz would not satisfy the boundary conditions.
} 
to

$$
\begin{aligned}
& \eta_{1}^{\bar{a}_{1}} \in \Gamma\left(\phi^{*} \pi_{1}^{*} \bar{T}_{\mathcal{Y}_{1}}^{v}\right), \quad \rho_{1}^{a_{1}} \in \Gamma\left(\phi^{*} \pi_{1}^{*} T_{\mathcal{Y}_{1}}^{v} \otimes T_{\Sigma}^{*}\right), \quad \theta_{1 a_{1}} \in \Gamma\left(\phi^{*} \pi_{1}^{*} T_{\mathcal{Y}_{1}}^{v *}\right), \\
& \eta_{2}^{\bar{a}_{2}} \in \Gamma\left(\phi^{*} \pi_{2}^{*} \bar{T}_{\mathcal{Y}_{2}}^{v}\right), \quad \rho_{2}^{a_{2}} \in \Gamma\left(\phi^{*} \pi_{2}^{*} T_{\mathcal{Y}_{2}}^{v} \otimes T_{\Sigma}^{*}\right), \quad \theta_{2 a_{2}} \in \Gamma\left(\phi^{*} \pi_{2}^{*} T_{\mathcal{Y}_{2}}^{v *}\right) .
\end{aligned}
$$

The latter give rise to the 0 -form

$$
\eta^{\bar{i}} \in \Gamma\left(\phi^{*} \bar{T}_{Y}\right)
$$

the 1 -form

$$
\rho^{i} \in \Gamma\left(\phi^{*} T_{Y} \otimes T_{\Sigma}^{*}\right)
$$

which is the constant Fourier mode of the component of $\chi^{i}$ along $\Sigma$, and the 0 -form

$$
\theta_{i}=\int \Omega_{i j^{\prime}} \chi_{3}^{j^{\prime}} d x^{3} \in \Gamma\left(\phi^{*} T_{Y}^{*}\right)
$$

Their BRST transformations are

$$
\begin{array}{ll}
\delta_{Q, W} \phi_{1}^{\bar{a}_{1}}=\eta_{1}^{\bar{a}_{1}}+V_{1 \bar{i}}^{\bar{a}_{1}} \eta^{\bar{i}}, & \delta_{Q, W} \phi_{1}^{a_{1}}=0, \\
\delta_{Q, W} \eta_{1}^{\bar{a}_{1}}=-\left(\partial_{\bar{b}_{1}} V_{1 \bar{i}}^{\bar{a}_{1}}\right) \eta_{1}^{\bar{b}_{1}} \eta^{\bar{i}}+\frac{1}{2} \mathcal{R}_{1 \bar{j}}^{\bar{a}_{1}} \eta^{\bar{i}} \eta^{\bar{j}}, & \delta_{Q, W} \theta_{1 a_{1}}=-\partial_{a_{1}} W_{1}, \\
\delta_{Q, W} \rho_{1}^{a_{1}}=\nabla \phi_{1}^{a_{1}}-\mathcal{R}_{1 i \bar{j}}^{a_{1}} \eta^{\bar{j}} \rho^{i}-\left(\partial_{\bar{b}_{1}} V_{1 i}^{a_{1}}\right) \eta_{1}^{\bar{b}_{1}} \rho^{i}, & \\
\delta_{Q, W} \phi_{2}^{\bar{a}_{2}}=\eta_{2}^{\bar{a}_{2}}+V_{2 \bar{i}}^{\bar{a}_{2}} \eta^{\bar{i}}, & \delta_{Q, W} \phi_{2}^{a_{2}}=0, \\
\delta_{Q, W} \eta_{2}^{\bar{a}_{2}}=-\left(\partial_{\bar{b}_{2}} V_{2 \bar{i}}^{\bar{a}_{2}}\right) \eta_{2}^{\bar{b}_{2}} \eta^{\bar{i}}+\frac{1}{2} \mathcal{R}_{2 \bar{i}}^{\bar{a}_{2}} \eta^{\bar{i}} \eta^{\bar{j}}, & \delta_{Q, W} \theta_{2 a_{2}}=\partial_{a_{2}} W_{2}, \\
\delta_{Q, W} \rho_{2}^{a_{2}}=\nabla \phi_{2}^{a_{2}}-\mathcal{R}_{2 i \bar{j}}^{a_{2}} \eta^{\bar{j}} \rho^{i}-\left(\partial_{\bar{b}_{2}} V_{2 i}^{a_{2}}\right) \eta_{2}^{\bar{b}_{2}} \rho^{i}, & \delta_{Q} \eta^{\bar{i}}=0, \\
\delta_{Q, W} \rho^{i}=d \phi^{i}, & \\
103) \delta_{Q, W} \theta_{i}=\left(\partial_{\bar{b}_{1}} V_{1 i}^{a_{1}} \eta_{1}^{\bar{b}_{1}}+\mathcal{R}_{1 i \bar{j}}^{a_{1}} \eta^{\bar{j}}\right) \theta_{1 a_{1}}+\left(\partial_{\bar{b}_{2}} V_{2 i}^{a_{2}} \eta_{2}^{\bar{b}_{2}}+\mathcal{R}_{2 i \bar{j}}^{a_{2}} \eta^{\bar{j}}\right) \theta_{2 a_{2}} & -\left(\partial_{i} W_{1}+V_{1 i}^{a_{1}} \partial_{a_{1}} W_{1}\right)+\left(\partial_{i} W_{2}+V_{2 i}^{a_{2}} \partial_{a_{2}} W_{2}\right) .
\end{array}
$$

These transformations look complicated, but this is an artefact of using a nonholomorphic trivialization of the tangent bundle of $\mathcal{Y}_{1} \times_{Y} \mathcal{Y}_{2}$. If we assemble the fields $\eta_{1}^{\bar{a}_{1}}, \eta_{2}^{\bar{a}_{2}}, \eta^{\bar{i}}$ into a single field $\eta^{\bar{A}} \in \Gamma\left(\phi^{*} \bar{T}_{\mathcal{Y}_{1} \times_{Y} \mathcal{Y}_{2}}\right)$, the fields $\rho_{1}^{a_{1}}, \rho_{2}^{a_{2}}, \rho^{i}$ into a single field $\rho^{A} \in \Gamma\left(\phi^{*} T_{\mathcal{Y}_{1} \times_{Y} \mathcal{Y}_{2}} \otimes T_{\Sigma}^{*}\right)$ and the fields $\theta_{1 a_{1}}, \theta_{2 a_{2}}, \theta_{i}$ into a single field $\theta_{A} \in \Gamma\left(\phi^{*} T_{\mathcal{Y}_{1} \times_{Y} \mathcal{Y}_{2}}^{*}\right)$, and use the 
holomorphic coordinate trivialization, we find

$$
\begin{aligned}
\delta_{Q, W} \phi^{\bar{A}} & =\eta^{\bar{A}}, & & \delta_{Q, W} \phi^{A}=0, \\
\delta_{Q, W} \eta^{\bar{A}} & =0, & & \delta_{Q, W} \theta_{A}=-\partial_{A}\left(W_{1}-W_{2}\right), \\
\delta_{Q, W} \rho^{A} & =d \phi^{A} . & &
\end{aligned}
$$

These are the BRST transformations of the curved B-model with target $\mathcal{Y}_{1} \times_{Y} \mathcal{Y}_{2}$ and the curving $\pi_{1}^{*} W_{1}-\pi_{2}^{*} W_{2}$. Presumably, quantum corrections further deform this result.

2.7.4. Different submanifolds, trivial fibrations. Now let us allow $Y_{1}$ and $Y_{2}$ to be different, but assume that there are no boundary degrees of freedom, i.e. $\mathcal{Y}_{1}=Y_{1}$ and $\mathcal{Y}_{2}=Y_{2}$. The classical vacua of the RW theory on $S^{1} \times \Sigma$ are constant field configurations satisfying the boundary conditions. Since the left and right boundaries must be mapped to $Y_{1}$ and $Y_{2}$, respectively, the set of classical vacua is $Y_{12}=Y_{1} \cap Y_{2}$. An obvious guess for a $2 \mathrm{~d}$ field theory with this set of classical vacua is the B-model with target $Y_{12}$. However, if $Y_{12}$ is not an embedded submanifold, it is not clear what this means. Even if $Y_{12}$ is an embedded submanifold (e.g. when $Y_{12}$ consists of several isolated points), the naive guess is not always correct. This happens because in general the directions transverse to $Y_{12}$ do not correspond to massive degrees of freedom.

To make a more intelligent guess, let $\Sigma=S^{1} \times \mathbb{R}$. Then one can determine the quantum space of states by first reducing the RW theory on $S^{1}$. As discussed above, reduction on $S^{1}$ yields a B-model with target $X$ on $I \times \mathbb{R}$. The boundary conditions correspond to Lagrangian submanifolds $\iota_{1}: Y_{1} \hookrightarrow X$ and $\iota_{2}: Y_{2} \hookrightarrow X$. The space of states of this B-model is

$$
\oplus_{p} \operatorname{Ext}_{X}^{p}\left(\iota_{1 *} \mathcal{O}_{Y_{1}}, \iota_{2 *} \mathcal{O}_{Y_{2}}\right) .
$$

More precisely, this is the space of boundary-changing operators. Let us compute this space when $Y_{12}=Y_{1} \cap Y_{2}$ consists of a single point $r \in X$. In the neighborhood of $r$ we can choose complex Darboux coordinates $p_{i}, q^{i}$ so that $\Omega=d q_{i} d p^{i}, Y_{1}$ is given by the equations

$$
p_{i}=\frac{\partial F_{1}(q)}{\partial q^{i}}
$$

and $Y_{2}$ is given by the equations

$$
p_{i}=\frac{\partial F_{2}(q)}{\partial q^{i}},
$$


for some holomorphic functions $F_{1}(q), F_{2}(q)$ on an open set $U$ in a vector space $V \simeq \mathbb{C}^{n}$. The functions $F_{1}, F_{2}$ are called generating functions of the Lagrangians $Y_{1}$ and $Y_{2}$. The intersection of $Y_{1}$ and $Y_{2}$ is the critical set of $F_{1}-F_{2}$; by assumption $r \in U$ is the only critical point of $F_{1}-F_{2}$ in $U$. Given a choice of Darboux coordinates, we may identify an open neighborhood of $r$ with an open neighborhood of the zero section in $T_{U}^{*}$ and regard the intersections of $Y_{1}$ and $Y_{2}$ with this neighborhood as Lagrangian submanifolds of $T_{U}^{*}$.

It is obvious physically and can be proved mathematically that the Ext groups depend only on the behavior of $Y_{1}$ and $Y_{2}$ in an arbitrarily small open neighborhood of $r$. Therefore to compute the Ext groups we can use a local Koszul resolution for a submanifold $Y_{1}$ of $T_{U}^{*}$ :

$$
\Lambda^{n} V \otimes \mathcal{O} \stackrel{\alpha}{\longrightarrow} \Lambda^{n-1} V \otimes \mathcal{O} \stackrel{\alpha}{\longrightarrow} \cdots \stackrel{\alpha}{\longrightarrow} V \otimes \mathcal{O} \stackrel{\alpha}{\longrightarrow} \mathcal{O},
$$

where $\mathcal{O}=\mathcal{O}_{T_{U}^{*}}$, and $\alpha$ is a contraction with a section of $V^{*} \otimes \mathcal{O}$ given by $\left(p_{i}-\partial_{i} F_{1}\right) d q^{i}$. Applying $\operatorname{Hom}\left(-, \iota_{2 *} \mathcal{O}_{Y_{2}}\right)$ to the Koszul resolution, we get a complex

$$
\Lambda^{n} V^{*} \otimes \Gamma(U) \stackrel{\beta}{\longleftarrow} \cdots \stackrel{\beta}{\longleftarrow} V^{*} \otimes \Gamma(U) \stackrel{\beta}{\longleftarrow} \Gamma(U),
$$

where $\beta$ is exterior product with an element of $V^{*} \otimes \Gamma(U)$ given by $\left(\partial_{i} F_{2}-\partial_{i} F_{1}\right) d q^{i}$, and $\Gamma(U)=H^{0}\left(U, \mathcal{O}_{U}\right)$. The cohomology of this complex is nonvanishing only in the left-most term and is isomorphic to the vector space

$$
\Lambda^{n} V \otimes(\Gamma(U) / A)
$$

where $A$ is the ideal generated by partial derivatives of $F_{2}-F_{1}$. The factor $\Lambda^{n} V$ is unimportant, since the Lagrangian submanifolds $Y_{1}$ and $Y_{2}$ are equipped with holomorphic volume forms, which give a natural isomorphism $\Lambda^{n} V \simeq \mathbb{C}$.

The vector space $\Gamma(U) / A$ is the Jacobi ring of $F_{1}-F_{2}$ and is most naturally obtained in the Landau-Ginzburg model with target $U$ and superpotential $W=F_{1}-F_{2}$. This suggests that reduction on the interval produces such a Landau-Ginzburg model.

If $n$ is odd, the vector space (104) is purely odd. The same is also true for the space of states of the Landau-Ginzburg model with an odd-dimensional target. In fact, the choice of absolute grading in both cases is ambiguous: if instead of Ext groups one considers Tor groups, and instead of the space of states for the Landau-Ginzburg model one considers the space of local operators, one gets the Jacobi ring of $F_{1}-F_{2}$ with even grading for all $n$. This ambiguity is discussed in more detail in section 2.7.6.

We will now argue that when $Y_{1}$ and $Y_{2}$ intersect at an isolated point, the RW theory on $I \times \Sigma$ reduces to the Landau-Ginzburg model on $U$ 
with the superpotential $F_{1}-F_{2}$. We have seen above that this is true in the case $Y_{1}=Y_{2}$. In general, we observed in subsection 2.4 that a deformation of the Lagrangian submanifold by means of a generating function $F$ is equivalent, to first order in $F$, to adding a boundary superpotential $F$ and modifying the BRST transformation of $\chi_{3}^{k^{\prime}}$ by boundary terms:

$$
\delta_{Q} \chi_{3}^{k^{\prime}}=\partial_{3} \phi^{k^{\prime}}-\delta\left(x_{3}\right) \Omega^{k^{\prime} j} \partial_{j} F_{1}+\delta\left(x_{3}-1\right) \Omega^{k^{\prime} j} \partial_{j} F_{2} .
$$

Hence to first order in $F_{1}, F_{2}$ the field $\theta_{i}$ defined by (94) transforms as

$$
\delta_{Q} \theta_{i}=-\partial_{i}\left(F_{1}-F_{2}\right) .
$$

This is exactly the right transformation law for the Landau-Ginzburg model with superpotential $F_{1}-F_{2}$. After dropping the term involving $\partial_{3} \phi^{i^{\prime}}$, the boundary action (25) gives the following contribution to the action of the $2 \mathrm{~d}$ field theory on $\Sigma$ :

$$
\int_{\Sigma} \frac{1}{2} \hat{\nabla}_{i} \partial_{j}\left(F_{1}-F_{2}\right) \rho^{i} \rho^{j}
$$

This is the Landau-Ginzburg deformation of the B-model action with the superpotential $W=F_{1}-F_{2}$ (see section $\mathrm{B}$ for a brief review of the Landau-Ginzburg model). This proves our statement to leading order in $F_{1}, F_{2}$.

To complete the argument, we use the ghost number symmetry. We note that $F_{1}$ and $F_{2}$ both have weight 2 under this symmetry, just like $\Omega$. The fields $\eta$ and $\theta$ have weights +1 , while the fields $\rho$ have weight -1 . Terms of higher order in $F_{1}, F_{2}$ must be at least quartic in $\rho$. But since $\rho$ is a 1 -form and the action must be a 2 -form on $\Sigma$, no such terms are possible. ${ }^{6}$

Next we generalize this result to the case when $Y_{12}=Y_{1} \cap Y_{2}$ is arbitrary. For simplicity we will neglect the quantum corrections arising from the nontrivial external geometry of $Y_{12}$. Without loss of generality we may assume that $Y_{12}$ is connected. We choose an open neighborhood $K$ of $Y_{12}$ and a Lagrangian submanifold $U$ of $K$ containing $Y_{12}$. We require that in the neighborhood of any $r \in Y_{12}$ there exist Darboux coordinates $p_{i}, q^{i}, i=1, \ldots, n$ so that $U$ is given by the equations $p_{i}=$ $0, i=1, \ldots, n$, and Lagrangian submanifolds $Y_{1} \subset K$ and $Y_{2} \subset K$ are described by generating functions $F_{1}$ and $F_{2}$. Obviously, the generating functions $F_{1}, F_{2}$ are constant on $Y_{12}$.

\footnotetext{
${ }^{6}$ The topological character of the theory implies that the part of the action which is not BRST-exact cannot depend on the metric on $\Sigma$.
} 
As before, locally the reduction of the RW model gives the LandauGinzburg model with target $U$ and the superpotential $W=F_{1}-F_{2}{ }^{7}$ Unlike in the previous case, the critical points of this superpotential are not necessarily isolated; rather, the critical set is $Y_{12}$. It remains to check that $F_{1}-F_{2}$ is a globally-defined holomorphic function on $U$. We note that $F_{1}$ and $F_{2}$ are defined up to an additive constant, so $d\left(F_{1}-\right.$ $\left.F_{2}\right)$ is a well-defined closed holomorphic 1-form on $U$. We also know that this form vanishes on $Y_{12} \subset U$ and therefore its class in $H^{1}\left(Y_{12}\right)$ vanishes as well. By taking a sufficiently small $U$, we can always ensure that the restriction map $H^{1}(U) \rightarrow H^{1}\left(Y_{12}\right)$ is an isomorphism; then $d\left(F_{1}-F_{2}\right)$ can be integrated to a holomorphic function on $U$ defined up to an additive constant.

This answer simplifies in the case when $Y_{12}$ is a submanifold of $X$ and the intersection of $Y_{1}$ and $Y_{2}$ is clean. This means that for any $r \in Y_{12}$ we have

$$
T_{r} Y_{1} \bigcap T_{r} Y_{2}=T_{r} Y_{12}
$$

This implies that $F_{1}-F_{2}$ are Morse-Bott functions on $U$, with $Y_{12}$ being the critical submanifold. As explained in section $B$, in such a situation the Landau-Ginzburg model with target $U$ and superpotential $F_{1}-F_{2}$ is equivalent to the B-model with target $Y_{12}$. More precisely, this is true if $n-\operatorname{dim} Y_{12}$ is even; if $n-\operatorname{dim} Y_{12}$ is odd, the Landau-Ginzburg model with target $U$ and superpotential $F_{1}-F_{2}$ is equivalent to the LandauGinzburg model with target $Y_{12} \times \mathbb{C}$ and superpotential $x^{2}$, where $x$ is an affine coordinate on $\mathbb{C}$. (While the Landau-Ginzburg model with target $\mathbb{C}$ and superpotential $x^{2}$ is trivial on the closed worldsheet $\Sigma$, this is not true when $\Sigma$ has boundaries, see section B.2).

2.7.5. Different submanifolds, nontrivial fibrations. The most general case is a combination of all of the above. We have two fibrations $\mathcal{Y}_{1}$ and $\mathcal{Y}_{2}$ over complex Lagrangian submanifolds $Y_{1}$ and $Y_{2}$ whose intersection is a not-necessarily-smooth $Y_{12}$. For simplicity when discussing reduction we will neglect quantum corrections coming from the nontrivial external geometry of $Y$. We also need to choose an open neighborhood $K \supset Y_{12}$ and a Lagrangian submanifold $U$ containing $T$ and contained in $K$ such that locally there exist Darboux coordinates such that $U$ is given by the equation $p_{i}=0$, while $Y_{1} \bigcap K$ and $Y_{2} \bigcap K$ are given by the equations $p_{i}=\partial_{i} F_{1}$ and $p_{i}=\partial_{i} F_{2}$. The differential $d\left(F_{1}-F_{2}\right)$ is a globally-defined closed holomorphic 1-form on $U$ which can be integrated to a holomorphic function on $U$ defined up to an additive constant.

\footnotetext{
${ }^{7}$ Quantum corrections could deform this result.
} 
As before, if we neglect quantum corrections, one can replace $K$ with an infinitesimal neighborhood of the zero section of $T_{U}^{*}$ which we continue to call $K$. We have the projection $\kappa: T_{U}^{*} \rightarrow U$ which identifies $Y_{1} \cap K$ and $Y_{2} \bigcap K$ with $U$. We can use $\kappa$ to push the fibrations $\mathcal{Y}_{1}$ and $\mathcal{Y}_{2}$ to $U$; we will call the resulting fibrations $\mathcal{U}_{1}$ and $\mathcal{U}_{2}$. The curvings $W_{1}$ and $W_{2}$ are push forward to curvings $W_{\mathcal{U}_{1}}$ and $W_{\mathcal{U}_{2}}$ on $\mathcal{U}_{1}$ and $\mathcal{U}_{2}$, respectively.

We can view $Y_{s}$ equipped with a fibration $\mathcal{Y}_{s}$ as a deformation of $U$ equipped with $\mathcal{U}_{s}$. The deformations are described by generating functions $F_{s}, s=1,2$. If we neglect the deformations altogether, the reduced theory will be the curved B-model with target $\mathcal{U}_{1} \times_{U} \mathcal{U}_{2}$ and curving $\pi_{1}^{*} W_{\mathcal{U}_{1}}-\pi_{2}^{*} W_{\mathcal{U}_{2}}$, where $\pi_{s}, s=1,2$ denotes the projection from $\mathcal{U}_{1} \times_{U} \mathcal{U}_{2}$ to $\mathcal{U}_{s}$. To first order in $F_{1}, F_{2}$, we get an extra piece in the transformation law for $\theta_{i}$ :

$$
\tilde{\delta}_{Q, W} \theta_{i}=\delta_{Q, W} \theta_{i}-\partial_{i}\left(F_{1}-F_{2}\right),
$$

where $\delta_{Q, W}$ denotes the BRST-variation (103). Then in the holomorphic coordinate trivialization we get

$$
\tilde{\delta}_{Q, W} \theta_{A}=-\partial_{A}\left(W_{1}+F_{1}-W_{2}-F_{2}\right) .
$$

This is the BRST transformation for the curved B-model with the target $\mathcal{U}_{1} \times_{U} \mathcal{U}_{2}$ and the curving

$$
\pi_{1}^{*} W_{\mathcal{U}_{1}}-\pi_{2}^{*} W_{\mathcal{U}_{2}}+p_{12}^{*}\left(F_{1}-F_{2}\right),
$$

where $p_{12}$ is the projection $\mathcal{U}_{1} \times{ }_{U} \mathcal{U}_{2} \rightarrow U$. The modification of the boundary action due to $F_{1}, F_{2}$ gives the following correction to the action of the reduced theory:

$$
\int_{\Sigma} \frac{1}{2} \hat{\nabla}_{i} \partial_{j}\left(F_{1}-F_{2}\right) \rho^{i} \rho^{j}
$$

This is the Landau-Ginzburg deformation corresponding to the superpotential $p_{12}^{*}\left(F_{1}-F_{2}\right)$.

When considering reduction on $S^{1} \times \Sigma$, we found that for a curved worldsheet $\Sigma$ the action of the reduced theory contains a dilatonic coupling not usually present in the B-model. Such terms could also arise in the case of reduction on the interval. We leave the analysis of such terms to future work.

2.7.6. The grading ambiguity. The above discussion of dimensional reduction brings out a subtlety in the quantization of the RW model with boundaries: the absolute $\mathbb{Z}_{2}$-grading on the space of states is not well-defined if the complex dimension of $X$ is not divisible by four, i.e. 
if $n$ is odd. The underlying reason is that for odd $n$ there is no completely canonical choice of a BRST-invariant measure on the space of $\chi$ zero modes. For simplicity, let us assume that the boundary condition on $\partial M$ is given by a complex Lagrangian submanifold $Y$; then the zero modes of $\chi$ normal to $Y$ take values in $H^{1}(M, \partial M)$, and the zero modes tangent to $Y$ take values in $H^{1}(M)$. The BRST-invariant measure involves volume forms on $H^{1}(M)$ and $H^{1}(M, \partial M)$ raised to the $n^{\text {th }}$ power. If $n$ is odd, changing the orientation of $H^{1}(M)$ or $H^{1}(M, \partial M)$ results in a change of sign of the BRST-invariant measure.

One can explain this ambiguity in a different way. Let $\Sigma$ be an oriented 2-manifold with a boundary. Given a complex Lagrangian submanifold $Y$, the RW model attaches to $\Sigma$ a vector space with a well-defined relative $\mathbb{Z}_{2}$ grading. To define an absolute grading, it is sufficient to specify a distinguished vector whose grading is defined to be even. One can attempt to define such a distinguished vector by picking an oriented 3-manifold $M$ whose boundary is split by a 1manifold into $\Sigma$ and $\Sigma^{\prime}$, where $\Sigma^{\prime}$ is some oriented 2-manifold with the same boundary as $\Sigma$. Given such $M$, one can consider the path-integral for the RW model on $M$ with the boundary condition corresponding to $Y$ specified on $\Sigma^{\prime}$. This gives a state in the vector space associated to $\Sigma$. This procedure works if different choices of $M$ produce vectors which have the same relative grading. For odd $n$ it turns out that this is not true, and consequently there is no canonical choice of absolute grading.

As an example, consider $\Sigma=S^{1} \times I$, where the boundary conditions on the two components of the boundary are identical and given by a Lagrangian submanifold $Y$ in $X$. If we reduce the theory on a circle, we obtain a B-model with target $X$ on an interval, with boundary conditions corresponding to a B-brane $Y$. Its space of states is $H^{\bullet}\left(\Lambda^{\bullet} N_{Y}\right)$. It is a Frobenius algebra; in particular, it has a trace function which is nonvanishing only on the component $H^{n}\left(\Lambda^{n} N_{Y}\right)$, where $n=\operatorname{dim}_{\mathbb{C}} Y$. It is natural to regard this component as even; this determines an absolute $\mathbb{Z}_{2}$ grading. From the three-dimensional viewpoint, this absolute grading corresponds to taking the 3 -manifold $M$ to be a solid torus whose boundary $S^{1} \times S^{1}$ is glued from two annuli $\Sigma$ and $\Sigma^{\prime}$.

On the other hand, if we reduce the theory on an interval, we obtain a B-model with target $Y$ on a circle. Its space of states is $H^{\bullet}\left(\Lambda^{\bullet} T_{Y}\right)$. It is again a Frobenius algebra, with a trace function which is nonvanishing only on the component $H^{n}\left(\Lambda^{n} T_{Y}\right)$. It is therefore natural to regard this component as even. From the three-dimensional viewpoint this definition of absolute grading corresponds to $M$ being a solid cylinder whose boundary is glued from an annulus $\Sigma$ and two disks. 
Now, since $Y$ is Lagrangian, we have $N_{Y} \simeq T_{Y}^{*}$, and since $Y$ is a Calabi-Yau manifold, we have $\Lambda^{p} N_{Y} \simeq \Lambda^{n-p} T_{Y}$. Thus the two vector spaces are naturally isomorphic as ungraded vector spaces, but the "natural" $\mathbb{Z}_{2}$ gradings agree only for even $n$.

Note that when $\Sigma$ has no boundary, the grading ambiguity does not arise [32]. While there is more than one way to "fill" $\Sigma$ with a 3manifold $M$ such that $\partial M=\Sigma$, all these ways correspond to the same grading on the vector space attached to $\Sigma$.

\section{Topological DEFECTS}

3.1. Surface operators. A surface operator in a $3 \mathrm{~d}$ TFT is a topological defect of codimension 1 . One can reduce to a large extent the study of surface operators to a study of boundary conditions using the "folding trick": if the location of the surface operator is given by $x^{3}=0$, one can identify the region $x^{3}<0$ with $x^{3}>0$ by means of the parity-reversing involution $x^{3} \mapsto-x^{3}$ and regard the surface operator as a boundary condition for a TFT which is a product of the original TFT and the parity-reversed TFT.

In the case of the RW model, parity-reversal is equivalent to replacing $\Omega$ with $-\Omega$. If $X$ is a complex symplectic manifold with a holomorphic symplectic form $\Omega$, let $X^{*}$ denote the same complex manifold with the holomorphic symplectic form $-\Omega$. Thus a surface operator for the RW model with target $X$ is the same as a boundary condition for the RW model with target $X^{*} \times X$.

Among all surface operators, there is a special one, corresponding to the diagonal $\Delta \subset X^{*} \times X$. It is obviously a Lagrangian submanifold, thanks to the relative minus sign between the symplectic forms of the two factors. The boundary conditions for this surface operator say that all fields and the first derivatives of bosonic fields vary continuously across $x^{3}=0$. Such a surface operator is equivalent to no surface operator at all, so we will refer to it as the invisible surface operator.

One can generalize the notion of a surface operator by considering the situation when the TFTs for $x^{3}>0$ and $x^{3}<0$ are not necessarily isomorphic. For example, they could be RW models with different target spaces $X_{1}$ and $X_{2}$. Such a surface operator is equivalent to a boundary condition for the RW model with target $X_{1}^{*} \times X_{2}$. The usual boundary condition is a special case of such a generalized surface operator corresponding to $X_{1}=\{p t\}, X_{2}=X$.

One important difference between boundary conditions and surface operators is that the set of surface operators has a monoidal structure (i.e. an associative multiplication with a unit object). Indeed, if we 

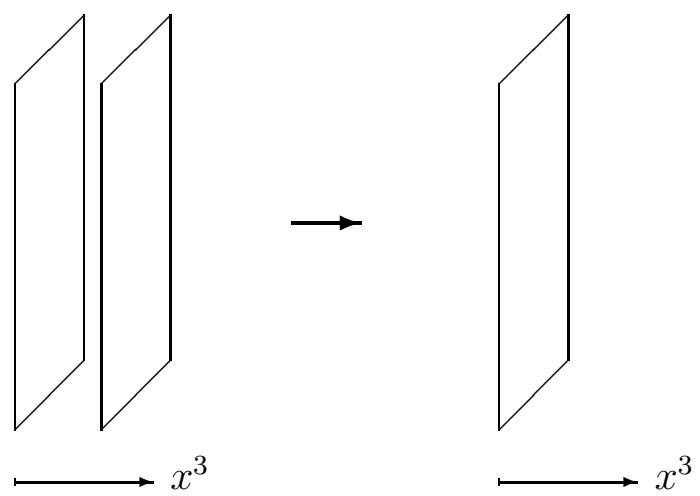

FiguRE 1. Fusion of surface operators.

consider two surface operators located at $x^{3}=0$ and $x^{3}=\epsilon$, by taking the limit $\epsilon \rightarrow 0+$ one gets another surface operator (Fig. 3.1). In the topological theory it is not really necessary to take the limit, since changing $\epsilon$ can be effected by a diffeomorphism of $M$. The invisible surface operator is the identity object for this monoidal structure.

One can also compose generalized surface operators, if we regard them as morphisms in a category whose objects are holomorphic symplectic manifolds, so we only consider composition when the "target" of the first surface operator is the same as the "source" of the second one. In fact, as explained below, the set of generalized surface operators between a fixed pair of holomorphic symplectic manifolds itself has the structure of a 2-category. Thus holomorphic symplectic manifolds are objects of a 3-category. This is discussed in more detail in [?].

In symplectic geometry one sometimes considers the "symplectic category" whose objects are symplectic manifolds and morphisms are Lagrangian correspondences, i.e. a morphism between $X_{1}$ and $X_{2}$ is a Lagrangian submanifold in $X_{1}^{*} \times X_{2}[39,3]$. This is not a true category because the composition of morphisms is defined only when Lagrangian submanifolds have a clean intersection [15, 38]. The category of surface operators on the other hand is well-defined by construction, i.e. compositions of morphisms are always defined. However, it is not easy to compute this composition in general. Neither is it obvious that the set of surface operators that we constructed is closed with respect to composition, although we believe this to be the case.

To compare the "symplectic category" and the category of surface operators, note that objects of the former are also objects of the latter. Now consider two Lagrangian correspondences $Y_{12} \subset X_{1}^{*} \times X_{2}$ and 
$Y_{23} \subset X_{2}^{*} \times X_{3}$. Let $\Delta_{X_{2}}$ denote the diagonal in $X_{2} \times X_{2}^{*}$. The composition $Y_{12} \circ Y_{23}$ in the "symplectic category" is defined as the image of

$$
\left(Y_{12} \times Y_{23} \bigcap X_{1}^{*} \times \Delta_{X_{2}} \times X_{3}\right) \subset X_{1}^{*} \times X_{2} \times X_{2}^{*} \times X_{3}
$$

under the projection to $X_{1}^{*} \times X_{3}$. When the intersection (105) is clean, its image is a Lagrangian submanifold in $X_{1}^{*} \times X_{3}[15,38]$. In general, the fiber of the projection over a point in the image may have dimension greater than zero. It is zero-dimensional precisely when the intersection is transverse.

On the other hand, we may consider the composition of $Y_{12}$ and $Y_{23}$ as generalized surface operators. The intersection (105) is precisely the space of classical vacua on $\Sigma=\mathbb{R}_{x_{1}, x_{2}}^{2}$ with the insertion of surface operators at $x_{1}=0$ and $x_{1}=\epsilon$. If the intersection is clean, the projection to $X_{1}^{*} \times X_{3}$ defines a fibration over its image whose fiber describes degrees of freedom living on the composite surface operator with the bulk fields fixed. Thus if the intersection is clean but not transverse, the composition of $Y_{12}$ and $Y_{23}$ as generalized surface operators is described not by the Lagrangian submanifold $Y_{12} \circ Y_{23}$, but by a fibration over it. Only in the case of transverse intersection do the two compositions agree.

We conclude this section by considering the composition of generalized surface operators in the case when the corresponding Lagrangian correspondences need not intersect cleanly. For simplicity, let $X_{1}=$ pt, $X_{2}=\mathbb{C}^{2 n}$ with coordinates $q^{1}, \ldots, q^{n}, p_{1}, \ldots, p_{n}, \Omega=d q^{i} d p_{i}$ and $X_{3}=\mathbb{C}^{2 n}$ with coordinates $\tilde{q}^{1}, \ldots, \tilde{q}^{n}, \tilde{p}_{1}, \ldots, \tilde{p}_{n}, \tilde{\Omega}=d \tilde{q}^{i} d \tilde{p}_{i}$. Suppose further that $Y_{12} \subset X_{2}$ is given by the generating function $f(q)$ and $Y_{23} \subset X_{2}^{*} \times X_{3}$ is given by the generating function $F(q, \tilde{q})$. Since $X_{1}=p t$, the generalized surface operator corresponding to $Y_{12}$ is simply a boundary condition for the RW model with target $X_{2}$. The fusion of the surface operator $Y_{23}$ with this boundary condition produces a new boundary condition for the RW model with target $X_{3}$.

Imagine now that the boundary is located at $x^{3}=0$, and the surface operator $Y_{23}$ is located at $x^{3}=\epsilon>0$. The degrees of freedom of the RW model with target $X_{2}$ on the interval $[0, \epsilon]$ should be thought of as boundary degrees of freedom for the new boundary condition. As for fields of the RW model with target $X_{3}$ on the half-line $x^{3}>\epsilon$, they should be kept fixed. Now we can appeal to the results of section 2.7 and conclude that the boundary degrees of freedom for the new boundary condition are described by a Landau-Ginzburg model with target $\mathbb{C}_{q}^{n}$ and the superpotential $W=f(q)-F(q, \tilde{q})$. This superpotential 


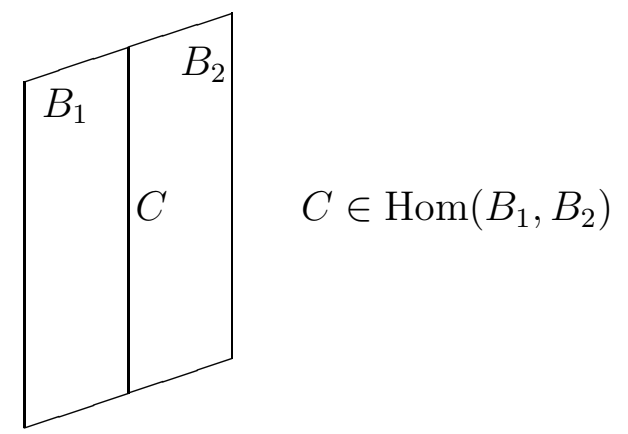

Figure 2. Line operator $C$ is a morphism between surface operators $B_{1}$ and $B_{2}$.

depends on the fields $\tilde{q}$, so in the end we get a trivial fibration over the Lagrangian submanifold $\mathbb{C}_{\tilde{q}}^{n}$ with fiber $\mathbb{C}_{q}^{n}$ and the superpotential $f(q)-F(q, \tilde{q})$.

\subsection{Line operators.}

3.2.1. Generalities. A line operator in a $3 \mathrm{~d} \mathrm{TFT}$ is a topological defect of codimension two. The corresponding one-dimensional submanifold can be in the interior of $M$, can split the boundary of $M$, or can split a two-dimensional submanifold on which a surface operator is inserted. The last case is the most general one, since one can regard a boundary line operator as a line operator on a surface operator between $X_{1}=\{p t\}$ and $X_{2}=X$, and one can regard the "bulk" line operator as a line operator on the invisible surface operator in $M$.

Note that the submanifold corresponding to a line operator may separate the "worldsheet" of the surface operator into two disconnected pieces, so that one may have different surface operators on the two sides of the line operator (Fig. 3.2.1). One may regard line operators as morphisms in a category whose objects are surface operators. Since surface operators themselves form a category, one can think of line operators as 2-morphisms in a 2-category whose objects are complex symplectic manifolds and whose 1-morphisms are surface operators. In fact, the set of line operators separating two fixed surface operators has the structure of a $\mathbb{C}$-linear category, with morphisms corresponding to point operators separating different line operators. Thus this 2category is really a 3-category. Its objects are holomorphic symplectic manifolds, its 1-morphisms are generalized surface operators, its 2morphisms are line operators on generalized surface operators, and its 3-morphisms are point operators. 


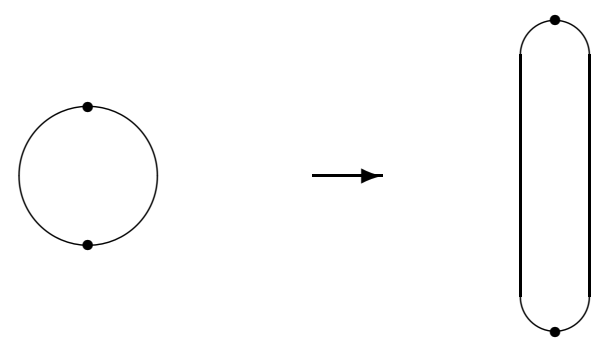

FiguRE 3. Boundary line operators in 3d TFT correspond to branes in the effective $2 \mathrm{~d}$ TFT obtained by reducing $3 \mathrm{~d}$ TFT on an interval.

3.2.2. Line operators on the boundary. The study of line operators can be reduced to a large extent to the study of branes in $2 \mathrm{~d}$ TFT. Without loss of generality, we may consider line operators on the boundary of $M$, since line operators on a surface separating RW models with targets $X_{1}$ and $X_{2}$ are equivalent to line operators on the boundary of the RW model with target $X_{1}^{*} \times X_{2}$. Let us further suppose that the line operator is a morphisms between boundary conditions corresponding to curved fibrations $\left(\mathcal{Y}_{1}, W_{1}\right)$ and $\left(\mathcal{Y}_{2}, W_{2}\right)$ over Lagrangian submanifolds $Y_{1}$ and $Y_{2}$ respectively. Let $M$ be $D^{2} \times \mathbb{R}$, where the unit disc $D^{2}$ is regarded as space and $\mathbb{R}$ as time. We will further assume that there are two marked points $r_{0}, r_{1}$ on $\partial D^{2}$, and two line operators located at $\left\{r_{0}\right\} \times \mathbb{R}$ and $\left\{r_{1}\right\} \times \mathbb{R}$. In the limit when $D^{2}$ becomes an infinitely narrow oval, the RW model on such $M$ reduces to a $2 \mathrm{~d}$ TFT on $I \times \mathbb{R}$, and the line operators become boundary conditions for this $2 \mathrm{~d}$ TFT (see Fig. 3.2.2). Point operators located on line operators become boundary-changing operators in the $2 \mathrm{~d}$ TFT, so the category of line operators is equivalent to the category of branes in the $2 \mathrm{~d}$ TFT.

In the case of the RW model, the effective $2 \mathrm{~d}$ TFT was determined in subsection 2.7. It is simple to describe it in the classical approximation in the case when $Y_{1}=Y_{2}=Y$. On the classical level the reduced theory is a curved B-model with target $\mathcal{Y}_{1} \times_{Y} \mathcal{Y}_{2}$ and the curving $\pi_{1}^{*} W_{1}-\pi_{2}^{*} W_{2}$, where $\pi_{s}$ is the projection from $\mathcal{Y}_{1} \times_{Y} \mathcal{Y}_{2}$ to $\mathcal{Y}_{s}$. In what follows we will not show the curving explicitly and will write $\mathcal{Y}_{s}$ instead of $\left(\mathcal{Y}_{s}, W_{s}\right)$. We will also denote by $\mathcal{Y}_{s}^{*}$ the pair $\left(\mathcal{Y}_{s}, W_{s}\right)$. Thus the category of branes in the effective $2 \mathrm{~d}$ TFT is the curved derived category of $\mathcal{Y}_{1} \times_{Y} \mathcal{Y}_{2}^{*}$. Here it was assumed that the orientation of the part of the boundary corresponding to $\mathcal{Y}_{1}$ agrees with the orientation of the line operator, so we should regard this category as the category of morphisms from the 1-object $\mathcal{Y}_{2}$ to the 1-object $\mathcal{Y}_{1}$. The category of morphisms from $\mathcal{Y}_{1}$ to $\mathcal{Y}_{2}$ is the curved derived category of $\mathcal{Y}_{1}^{*} \times_{Y} \mathcal{Y}_{2}$. 
If the class $[\beta] \in H^{1}\left(T_{Y} \otimes N_{Y}^{*}\right)$ is nonvanishing, quantum corrections may deform the target of the reduced theory; for example, as explained in section 2.7, without fibrations the leading deformation is described by the Beltrami differential $\mu=\frac{\beta}{2}\left(\partial W_{1}+\partial W_{2}\right)$. We also showed in section 2.7 that if $\mathcal{Y}_{1}=\mathcal{Y}_{2}=Y$, and $W_{1}=W_{2}=0$, the classical answer is exact. That is, in this special case the category of boundary line operators is exactly the $\mathbb{Z}_{2}$-graded derived category of $Y$.

Another simple case is when fibrations are absent and $Y_{1}$ and $Y_{2}$ intersect over a finite set $\left\{p_{1}, \ldots, p_{\ell}\right\} \subset X$. In this case the reduced $2 \mathrm{~d}$ TFT is a Landau-Ginzburg model whose target is a union of open sets $U_{k} \subset \mathbb{C}^{n}, k=1, \ldots, \ell$, with superpotentials $W_{1}, \ldots, W_{\ell}$. The superpotential $W_{k}$ on $U_{k}$ is determined by the local geometry of $Y_{1}$ and $Y_{2}$ in a neighborhood of $p_{k}$ : given a holomorphic symplectomorphism from this neighborhood to $T U_{k}^{*}, W_{k}$ is given by $F_{1}-F_{2}$, where $F_{1}$ and $F_{2}$ are the holomorphic generating functions on $U_{k}$ corresponding to $Y_{1}$ and $Y_{2}$, respectively.

Instead of utilizing the known results about branes in curved Bmodels, one can analyze the category of line operators on the boundary more directly. For definiteness, let us consider the case $Y_{1}=Y_{2}=Y$; as explained in subsection 2.7, this entails no real loss of generality, since the general case can be reduced to this one. Let us show explicitly how to construct a boundary line operator given an object of the curved derived category of $\mathcal{Y}_{1} \times_{Y} \mathcal{Y}_{2}^{*}$. For simplicity, we will do on the classical level, i.e. neglecting boundary corrections to BRST transformations originating from the second fundamental form of $Y$.

We consider a connected component $\Sigma$ of $\partial M$ and a one-dimensional submanifold $\gamma \subset \Sigma$ which splits $\Sigma$ into $\Sigma_{1}$ and $\Sigma_{2}$. We will assume that the orientation of $\gamma$ agrees with the orientation of $\Sigma_{1}$ and disagrees with the orientation of $\Sigma_{2}$. The boundary condition on $\Sigma_{s}$ is described by the fibration $\mathcal{Y}_{s}$, equipped with the curving $W_{s} \in H^{0,0}\left(\mathcal{Y}_{s}\right), s=1,2$. The BRST variation of the part of the boundary action coming from $\Sigma_{s}$ is

$$
\delta_{Q} S_{s}^{b r y}=\int_{\Sigma_{s}}\left(\Omega^{k^{\prime} i} A_{k^{\prime}}^{K}\left(\mathcal{F}_{s i \bar{A}}^{a} \theta_{a} H_{s}^{\bar{A}} \frac{\delta S^{b u l k}}{\delta \chi_{3}^{K}}+\nabla_{i} W_{s} \frac{\delta S^{b u l k}}{\delta \chi_{3}^{K}}\right)+d W_{s}^{(1)}\right),
$$

where $W_{s}^{(1)}$ is given by (87) with $W$ replaced with $W_{s}$. The BRST transformation of $\chi_{3}^{K}$ involves boundary terms as in (80); thanks to these boundary terms the BRST variation of the total action is

$$
\delta_{Q, W}\left(S_{1}^{b r y}+S_{2}^{b r y}+S^{b u l k}\right)=\int_{\gamma}\left(W_{1}^{(1)}-W_{2}^{(1)}\right) .
$$


Here $W_{1}-W_{2}$ is regarded as a $\bar{\partial}$-closed form of type $(0, \hat{0})$ on $\mathcal{Y}_{12}=$ $\mathcal{Y}_{1} \times_{Y} \mathcal{Y}_{2}$. This BRST variation must be canceled by the BRST variation of the line operator on $\gamma$.

Now consider an object of the curved derived category of $\mathcal{Y}_{12}$. It is a pair $(E, D)$, where $E$ is a smooth $\mathbb{Z}_{2}$-graded vector bundle on $\mathcal{Y}_{12}$ and $D$ is an odd differential operator on $\Omega^{0, \bullet}(E)$ satisfying (122). The restrictions of the fields $\phi^{A}, H^{\bar{A}}$ and $P^{A}$ to $\gamma$ define a map $\Phi: \gamma \rightarrow \Pi \bar{T}_{\mathcal{Y}_{12}^{*}}$ and a fermionic 1-form $\rho^{A}$ on $\gamma$ with values in the pull-back of $T_{\mathcal{Y}_{12}}$. The BRST operator $\delta_{Q}$ acts on the space of pairs $\left(\Phi, \rho^{A}\right)$. As explained in section B.2, given an object $(E, D)$ of the curved derived category of $\mathcal{Y}_{12}$ one can construct a function $\exp \left(-S^{\gamma}\right)$ on the space of pairs $\left(\Phi, \rho^{A}\right)$ whose BRST variation is the right-hand-side of (106). This function is the supertrace of the holonomy of a certain connection on $\Phi^{*}(E)$ constructed from $D$ and the fields $\Phi$ and $\rho^{A}$. We take $\exp \left(-S^{\gamma}\right)$ as the line operator corresponding to $(E, D)$.

The function $\exp \left(-S^{\gamma}\right)$ greatly simplifies in the case when $\mathcal{Y}_{1}=$ $\mathcal{Y}_{2}=Y$, and $(E, D)$ is a holomorphic vector bundle on $Y$. Then the line operator takes the form

$$
\operatorname{Tr} \operatorname{Hol}_{\gamma}\left(A_{i} d \phi^{i}+A_{\bar{i}} d \phi^{\bar{i}}+F_{i \bar{j}} \chi^{i} \eta^{\bar{j}}\right)
$$

where $\left(A_{i}, A_{\bar{i}}\right)$ are components of a connection 1 -form on $E$, and $F_{i \bar{j}}$ is the curvature of this connection.

3.2.3. Fusion of line operators on the boundary. In the case $\mathcal{Y}_{1}=\mathcal{Y}_{2}=$ $\mathcal{Y}$, the category of line operators is the category of endomorphisms of the object $\mathcal{Y}$ in the 2 -category of boundary conditions. Therefore it has a monoidal structure realized physically by fusing the line operators on the boundary. The invisible line operator is the unit object with respect to this monoidal structure. Mathematically, it is represented by the "fibered diagonal" $\Delta_{Y}$ in the fibered product $\mathcal{Y} \times_{Y} \mathcal{Y}$. Note that the curving vanishes on $\Delta_{Y}$, so presumably this is a valid object of the curved derived category (see [22] where this is explained in the special case of the Landau-Ginzburg model).

As explained above, to any line operator on the boundary described by the fibration $\mathcal{Y}$ one can associate a boundary condition in the curved B-model with target $\mathcal{Y}^{*} \times_{Y} \mathcal{Y}$ (classically) or its deformation (quantummechanically). From this viewpoint, the monoidal structure on the category of line operators is not natural. Nevertheless, there is a purely two-dimensional interpretation of this monoidal structure. Consider a a 3-manifold $M=\mathbb{R} \times \Sigma$ where $\Sigma$ is a disc. On the boundary of $M$ we specify a boundary condition described by $\mathcal{Y}$ and insert a line operator which is located at a point on the boundary of $\Sigma$. Consider squashing 


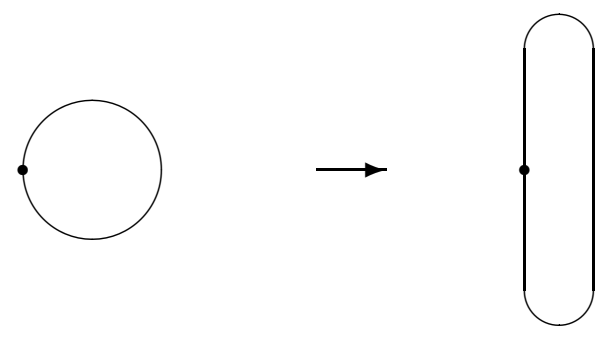

Figure 4. Boundary line operators in 3d TFT also correspond to bulk line operators in the effective $2 \mathrm{~d}$ TFT obtained by reducing $3 \mathrm{~d}$ TFT on an interval.

$\Sigma$ into an interval (see Fig. 3.2.3). According to section 2.7, the RW model reduces to the curved B-model on $I \times \mathbb{R}$ with target $\mathcal{Y}^{*} \times_{Y}$ $\mathcal{Y}$ (more precisely, its deformation), and the boundary line operator becomes a bulk line operator in the B-model located at a point in the interior of $I$. Fusing boundary line operators in the RW model obviously reduces to fusing bulk line operators in the B-model.

The category of bulk line operators in a curved B-model with target $Z$ is equivalent to the curved derived category of $Z^{*} \times Z$ where $Z^{*}$ denotes $Z$ with the opposite curving. The monoidal structure is given by the convolution product. Equivalently, an object of the curved derived category of $Z^{*} \times Z$ can be thought of as a functor from the curved derived category of $Z$ to itself, and the monoidal structure is given by the composition of such functors. This monoidal structure is not symmetric (i.e. it is noncommutative).

Bulk line operators of the reduced theory which originate from boundary line operators in the parent RW theory on an interval form a monoidal subcategory in the curved derived category of $Z^{*} \times Z$, where $Z$ is a deformation of $\mathcal{Y}^{*} \times_{Y} \mathcal{Y}$. In general, there is no reason to expect this monoidal subcategory to be symmetric.

The curved derived category of $\mathcal{Y}^{*} \times_{Y} \mathcal{Y}$ has an obvious monoidal structure given by the convolution product. Equivalently, we can think of an object of the curved derived category of $\mathcal{Y}^{*} \times_{Y} \mathcal{Y}$ as a functor from the curved derived category of $\mathcal{Y}$ to itself, and the obvious monoidal structure is given by the composition of such functors. It is easy to see that on the classical level this is the right monoidal structure. We will now argue that it receives quantum corrections which depend not only on the geometry of $\mathcal{Y}$, but also on the way $Y$ is embedded into $X$.

To simplify the discussion, we will consider in detail the case $\mathcal{Y}=Y$ and vanishing curving. In this case the category of boundary line operators is simply the $\mathbb{Z}_{2}$-graded derived category of $Y$, and the obvious 
monoidal structure is given by the tensor product of vector bundles on $Y$. This monoidal structure is symmetric and the unit object is the trivial rank-one bundle on $Y$. It is obvious that fusion of line operators on the classical level corresponds to tensoring the corresponding vector bundles. We would like to determine the leading quantum correction to this result.

The most straightforward way is to evaluate the product of boundary line operators in perturbation theory; in section $\mathrm{C}$ we do it to leading order in $\hbar$. In this section we will take an indirect route which is less computationally demanding. Let $(E, D)$ be an object of the $\mathbb{Z}_{2}$-graded derived category of $Y$, i.e. $E$ is a smooth $\mathbb{Z}_{2}$-graded vector bundle on $Y$ and $\mathcal{K}$ is an odd differential operator on $\Omega^{0, \bullet}(E)$ satisfying (122) with $W=0$. We can represent the operation of tensoring with $(E, D)$ by an object of the $\mathbb{Z}_{2}$-graded derived category of $Y \times Y$, namely the push-forward of $(E, D)$ from the diagonal in $Y \times Y$. An infinitesimal deformation of this functor corresponds to an infinitesimal deformation of the corresponding object on $Y \times Y$. The latter are classified by odd elements of the endomorphism vector space of $\delta_{*}(E, D)$, where $\delta$ is the diagonal embedding of $Y$ into $Y \times Y$. This space is isomorphic to the cohomology of $D$ on the space $\Omega^{0, \bullet}(\operatorname{End}(E)) \otimes \Lambda^{\bullet} T_{Y}$ (this is proved in the same way as in the usual derived category).

We can determine the leading-order deformation without any computations as follows. The boundary line operator corresponding to a pair $(E, D)$ is the holonomy of a certain superconnection $\mathcal{N}$ defined by (117). This superconnection depends on the restrictions of various fields to the 1-dimensional submanifold $\gamma \in \partial M$. Quantum corrections to the composition of line operators are obtained by expanding the holonomy of $\mathcal{N}$ in a power series in the fields and evaluating the contractions of fermionic and bosonic fields. The contractions arise from interactions and therefore must involve the curvature of $Y$, the second fundamental form of $Y \subset X$, and their derivatives. From the algebro-geometric viewpoint the curvature represents an element of $H^{1}\left(\Omega_{Y}^{1} \otimes \operatorname{End} T_{Y}\right)$ (the Atiyah class of the tangent bundle of $Y$ ), while the second fundamental form $\beta$ represents an element of $H^{1}\left(T_{Y} \otimes N_{Y}^{*}\right)$ determined by the exact sequence (23). For a Lagrangian submanifold one has $N_{Y}^{*} \simeq T_{Y}$, and $[\beta] \in H^{1}\left(\operatorname{Sym}^{2} T_{Y}\right)$. The leading term in the expansion of the holonomy of $\mathcal{N}$ is proportional to the curvature of $D$, i.e.

$$
F=\nabla D+D \nabla \in \Omega_{Y}^{1} \otimes \Omega^{0, \bullet}(\operatorname{End}(E))
$$

The form $F$ is $D$-closed and can be regarded as a representative of the Atiyah class of the object $(E, D)$ in the $\mathbb{Z}_{2}$-graded derived category. 
These considerations uniquely determine the leading deformation to be

$$
\beta\left\llcorner F \in T_{Y} \otimes \Omega^{0, \bullet}(\operatorname{End}(E)),\right.
$$

where $L$ denotes a contraction between a holomorphic bi-vector field and a holomorphic 1-form. This class is odd, as required, and $D$-closed (because $\beta$ is $\bar{\partial}$-closed and $F$ is $D$-closed). Thus it defines a leading deformation of the pushforward of $(E, D)$ from $Y$ to $Y \times Y$.

It remains to check whether the coefficient of the leading deformation is really nonzero (the normalization of the coefficient is unimportant). This is done in section $\mathrm{C}$ by evaluating the OPE of the Wilson loops to leading order in $\beta$. As a preparation, let us make the deformation of the monoidal structure a bit more explicit in the case when $(E, D)$ is a holomorphic vector bundle on $Y$. Then $F$ is the ordinary curvature of $E$, and the deformation corresponds to an element

$$
\left[\beta\llcorner F] \in H^{2}\left(T_{Y} \otimes \text { End } E\right) .\right.
$$

Thus if $E_{1}$ and $E_{2}$ are two holomorphic vector bundles on $Y$, the composition of the corresponding line operators will be the smooth vector bundle $E_{12}=E_{1} \otimes E_{2}$ equipped with a differential operator

$$
D_{12}=\bar{\partial}+\beta\left\llcorner\left(F_{1} \wedge F_{2}\right)\right.
$$

on $\Omega^{0, \bullet}\left(E_{1} \otimes E_{2}\right)$. Here $\bar{\partial}$ is the ordinary $\bar{\partial}$-operator on $E_{1} \otimes E_{2}$ and $F_{1}$ and $F_{2}$ are curvature forms of $E_{1}$ and $E_{2}$. Note that the quantum correction to $\bar{\partial}$ changes sign under the exchange of $E_{1}$ and $E_{2}$ which means that the deformed monoidal structure is not symmetric. Note also that the correction is a $(0,3)$ form on $Y$, so the composition of line operators corresponding to holomorphic line bundles is no longer a holomorphic line bundle but a more general object of the $\mathbb{Z}_{2}$-graded derived category of $Y$.

If the class $[\beta]$ vanishes, then to leading order in the Planck constant the monoidal structure of the category of boundary line operators is not deformed. However, it is likely that at higher orders in the Planck constant the monoidal structure will be affected by the geometry of higherorder infinitesimal neighborhoods of $Y$ in $X$. For example, at quadratic order in the Planck constant we have a class $\left[\beta^{\prime}\right] \in H^{1}\left(\mathrm{Sym}^{3} T_{Y}\right)$ which likely deforms the associativity morphism in the category of boundary line operators. We will see an example of this below.

To conclude this section we will show that the invisible line operator on $Y$ (i.e. the unit object in the category of boundary line operators on $Y$ ) does not receive quantum corrections. On the classical level, the unit object is the structure sheaf $\mathcal{O}_{Y}$. A quantum correction would correspond to an element $\mu \in H^{\hat{1}}\left(\mathcal{O}_{Y}\right)$ which has ghost number 1 . This 
element must be constructed from the curvature tensor of $Y$ and the classes $\beta^{(\ell)} \in H^{1}\left(\operatorname{Sym}^{\ell} T_{Y}\right)$. Suppose $\mu$ contains $m$ curvature tensors as well as classes $\beta^{\left(\ell_{i}\right)}, i=1, \ldots, N$. Ghost number symmetry implies

$$
m+N+\sum_{i}\left(2-2 \ell_{i}\right)=1 .
$$

Hence $N=m+1 \bmod 2$. This also ensures that the cohomological degree of $\mu$ is odd, as required.

Now we note that the action of the RW theory has a parity symmetry if we require all fields to be parity-even and $\Omega$ to be parity-odd. The boundary condition corresponding to $Y$ is parity-invariant, and so is the invisible line operator on the boundary. Thus the class $\mu$ must be parity-even. By definition, $\beta^{(\ell)}$ contains $\Omega^{-1}$ raised to the power $\ell-1$ and so its parity is $\ell-1 \bmod 2$. This implies

$$
\sum_{i} \ell_{i}=N \bmod 2
$$

and therefore

$$
\sum_{i} \ell_{i}=m+1 \bmod 2
$$

But then it is impossible to contract all holomorphic indices to get an element of $H^{\hat{1}}\left(\mathcal{O}_{Y}\right)$. Thus the invisible line operator is undeformed.

3.2.4. Line operators in the bulk. The monoidal 2-category of surface operators in the RW model has a unit object: the invisible surface operator. Its category of endomorphisms is the Hochschild cohomology of the 2-category of surface operators. From the physical viewpoint, it should be thought of as the category of line operators in the bulk.

Since the invisible surface operator is represented by the diagonal $\Delta \hookrightarrow X \times X$ with a trivial curving, the category of line operators in the bulk is the $\mathbb{Z}_{2}$-graded derived category of $X$. An object of this category can be represented by a $\mathbb{Z}_{2}$-graded smooth vector bundle $E$ on $X$ equipped with a differential operator $D$ on $\Omega^{0, \bullet}(E)$ satisfying $D^{2}=0$ and the Leibniz rule

$$
D(\omega \wedge \sigma)=\bar{\partial} \omega \wedge \sigma+(-1)^{\operatorname{deg}_{\mathbb{Z}_{2}} \omega} \omega \wedge D \sigma, \forall \omega \in \Omega^{0, \bullet}(X), \forall \sigma \in \Omega^{0, \bullet}(E) .
$$

The operator $D$ is called a $\bar{\partial}$-superconnection. One can get such an object from a complex of holomorphic vector bundles by letting $D=$ $\bar{\partial}+\mathcal{K}$, where $\mathcal{K}$ is the differential in the complex. This generalizes the observation of [32] that one can define a BRST line operator for any holomorphic vector bundle on $X$.

If we take $E$ to be trivial rank-one vector bundle, then the Wilson line operator is simply the identity operator, i.e. it is invisible. 
The category of bulk line operators has a monoidal structure obtained by fusing the operators together. The invisible line operator is the unit object; it corresponds to the structure sheaf $\mathcal{O}_{X}$. The second fundamental form for the diagonal in $X^{*} \times X$ vanishes, so to leading order in the Planck constant the monoidal structure is undeformed. But already at second order in the Planck constant we expect corrections, since the the second-order infinitesimal neighborhood of the diagonal is not isomorphic to the second-order infinitesimal neighborhood of the zero section in $T_{X}^{*}$ [18]. The deviation is parameterized by the Atiyah class of $T_{X}$, which can be regarded as an element of $H^{1}\left(\operatorname{Sym}^{3} T_{X}\right)$. Such a correction cannot affect the tensor product itself (i.e. cannot affect the isomorphism class of $E_{1} \otimes E_{2}$ ), but it can affect the associativity morphism which tells us how to identify $\left(E_{1} \otimes E_{2}\right) \otimes E_{3}$ and $E_{1} \otimes\left(E_{2} \otimes E_{3}\right)$. Thus at quadratic order in the Planck constant we expect a correction to the associativity morphism proportional to the contraction of this class with the Atiyah classes of the three objects involved.

The deformation of the associativity morphism is related to the fact that the category of bulk line operators is a braided monoidal one, with a nontrivial braiding $[32,31]$. The braided structure comes about because diffeomorphisms of a disc with marked points (at which line operators are inserted) act functorially on the category which TQFT associates to such a disk, and diffeomorphisms isotopic to identity act trivially. On the classical level, the braiding is trivial, but at linear order in the Planck constant a nontrivial braiding appears proportional to the Atiyah classes of the two objects involved [32, 31].

3.2.5. The categorified boundary-bulk map. In the $2 \mathrm{~d}$ TFT, there is a boundary-bulk map which sends an object of the category of branes to a local operator. This maps factors through the K-theory of the category of branes and can be regarded as a homomorphism from the K-theory to the additive group of the space of local operators. Pictorially, it is represented by a disc 1-correlator with an insertion of a bulk operator, or equivalently by an annulus whose inner boundary component is a cut boundary, and the outer boundary component is the brane boundary.

In $3 \mathrm{~d}$ TFT the same geometric structure corresponds to a map which sends an object of the 2-category of boundary conditions to a bulk line operator. One can interpret it as a categorified version of the Chern character. Let us determine it for boundary conditions in the RW model.

Consider first the case when the boundary condition is described by a complex Lagrangian submanifold $Y$. Let $(E, D)$ be an object of the 
$\mathbb{Z}_{2}$-graded derived category of $X$. We need to compute the vector space associated to a disc with an insertion of the bulk line operator $(E, D)$ and the boundary condition $Y$. The bosonic zero modes parameterize $Y$, while the fermionic zero modes are components of $\eta$ tangent to $Y$. The Hilbert space of the zero modes is therefore the space of smooth sections of $\Omega^{0, \bullet}\left(\left.E\right|_{Y}\right)$, and the vector space associated to the punctured disc is the cohomology of $\Omega^{0, \bullet}\left(\left.E\right|_{Y}\right)$ with respect to $D$. This means that the line operator corresponding to $Y$ is simply the structure sheaf of $Y$ pushed forward to $X$.

To determine the answer for a fibration $\mathcal{Y}$ over $Y$, note that one can deform the disc so that it looks like the surface of a cigar. One can then imagine the circumference of the cigar shrink to zero size. In this limit the boundary condition corresponding to $Y$ reduces to a boundary condition in the B-model with target $X$, and therefore the boundary-bulk map is simply reduction on a circle. Reduction on a circle for boundary conditions in the RW model has been described in section 2.6. Namely, given a fibration $\mathcal{Y}$ with compact fibers we take the flat vector bundle on $Y$ given by the fiberwise de Rham cohomology and push it forward to $X$.

3.2.6. Line operators corresponding to submanifolds in the target space. In the case of the B-model one can define a boundary condition corresponding to an arbitrary complex submanifold $Y$ in the target space $X$. It is believed that the resulting brane corresponds to an object of the derived category of $X$ which is the pushforward of $\mathcal{O}_{Y}$ to $X$. Such an object has a resolution by locally free sheaves, and therefore is isomorphic to a certain complex of holomorphic vector bundles on $X$. Thus as far as the B-model is concerned, it is sufficient to consider branes corresponding to complexes of holomorphic vector bundles on $X$.

If $X$ is a complex symplectic manifold, we can use the resolution of $\iota_{*} \mathcal{O}_{Y}$ to construct a line operator in the RW model with target $X$. Different resolutions will correspond to isomorphic line operators, but this is far from obvious from our construction of line operators. It is interesting to ask whether in the RW model there is an alternative description of the same line operator which is manifestly independent of the choice of resolution. By analogy with the B-model, one expects that such a line operator will be defined by the condition that $\phi$ maps the support $\gamma$ of the line operator to $Y$.

In this section we will describe a candidate for such a line operator in the RW model. Since it appears unnatural to impose conditions on $\phi$ on a codimension-two submanifold, we will regularize the problem 
by excising a tubular neighborhood $\mathcal{M}$ of $\gamma$ and will define the line operator by imposing suitable boundary conditions on fields on the boundary of $M \backslash \mathcal{M}$.

We will use cylindrical coordinates in the neighborhood of $\gamma:(r, \varphi, t)$, $r \in[0, \infty), \varphi \in[0,2 \pi), t \in \mathbb{R}$ so that $\mathcal{M}$ is given by $r<r_{0}$. In a topological field theory, one can assume $r_{0}$ to be arbitrarily small, since the correlators do not depend on it. Keeping this in mind, we will impose conditions at $r=r_{0}$ which are nonlocal in the $\varphi$-direction but local in the $t$-direction. This nonlocality will not matter when we take $r_{0}$ to be very small.

Near $r=r_{0}$ we can expand each field into a Fourier series, for example $\phi^{I}=\sum_{n \in \mathbb{Z}} \phi_{n}^{I}(r, t) e^{i n \varphi}$, etc. To ensure that the limit $r_{0} \rightarrow 0$ is non-singular we will require

$\phi_{n}^{I}=0, \quad \phi_{n}^{\bar{I}}=0, \quad \eta_{n}^{\bar{I}}=0, \quad \Lambda \chi_{t, n}^{I}-\chi_{\varphi, n}^{I}=0, \quad n \neq 0, \quad I, \bar{I}=1, \ldots, 2 n$

where the parameter $\Lambda^{-1}$ has mass dimension one. These conditions do not depend on the submanifold $Y$.

To write down the conditions for the constant Fourier-modes, suppose that $Y$ is locally defined by the equations $\phi^{i^{\prime}}=0, i^{\prime}=k+1, \ldots, 2 n$. We may use $\phi^{i}, i=1, \ldots, k$, as local coordinates on $Y$. We will require (108)

$\phi_{0}^{i^{\prime}}=0, \quad \phi_{0}^{\bar{i}^{\prime}}=0, \quad \eta_{0}^{\bar{i}^{\prime}}=0, \quad \Lambda \chi_{t, 0}^{i^{\prime}}-\chi_{\varphi, 0}^{i^{\prime}}=0, \quad i^{\prime}, \bar{i}^{\prime}=k+1, \ldots, 2 n$,

$$
\pi_{i, 0}=0, \quad \pi_{\bar{i}, 0}=0, \quad g_{J \bar{i}} \chi_{r, 0}^{J}=0, \quad g_{J \bar{i}} \chi_{\varphi, 0}^{J}=0, \quad i, \bar{i}=1, \ldots, k .
$$

Here $\pi_{i}$ and $\pi_{\bar{i}}$ are canonical momenta for $\phi^{i}$ and $\phi^{\bar{i}}$, if regard $r$ as the time coordinate. The system of constraints (107-109) is BRST invariant and ensures that $\partial \mathcal{M}$ is mapped to the $k$-dimensional submanifold $Y$.

In particular, if $Y=X$, the limit $r_{0} \rightarrow 0$ of these conditions simply says that all fields are smooth at $r=0$. This means that this line operators is trivial, or invisible.

3.3. Point operators. Let us make a few remarks about point operators, i.e. operators localized at points. A more standard name for them is local operators. The most general case is when such an operator is inserted at a joining point of two line operators. The space of such operators is the space of morphisms in the category of line operators. As explained above, the categories of line operators arising in the RW model are all equivalent to curved derived categories of certain complex manifolds, so in this way we get a complete description of point operators and their algebra. Let us give a few examples. 
Consider first a boundary condition corresponding to a fibration $\mathcal{Y}$ over a Lagrangian submanifold $Y$. A point operator on such a boundary can be thought of as sitting on an invisible line operator, which is represented by the fibered diagonal in $\mathcal{Y} \times_{Y} \mathcal{Y}$. The space of such line operators is the endomorphism algebra of the structure sheaf of the fibered diagonal $\Delta_{Y}$ which on the classical level is isomorphic to

$$
\oplus_{r} \operatorname{Ext}^{r}\left(\mathcal{O}_{\Delta_{Y}}, \mathcal{O}_{\Delta_{Y}}\right) \simeq \oplus_{p, q} H^{p}\left(\Lambda^{q} T_{\mathcal{Y}}^{v}\right)
$$

As discussed above, there may be nontrivial quantum corrections to this result due to nontrivial external geometry of $Y$; thus (110) is merely the first term in a spectral sequence which converges to the quantum algebra of boundary observables.

In the special case $\mathcal{Y}=Y,(110)$ reduces to

$$
\oplus_{p} H^{p}\left(\mathcal{O}_{Y}\right)
$$

which agrees with section 2.4. In this case no quantum corrections are possible. Indeed, we have shown above that in this case neither the category of boundary line operators nor the unit object receive quantum corrections. Hence the endomorphism algebra of the unit object is also uncorrected.

Our last example is a point operator in the bulk, which can be thought of as sitting on an invisible line operator in the bulk. The category of bulk line operators is equivalent to the $\mathbb{Z}_{2}$-graded derived category of $X$. The invisible line operator corresponds to the structure sheaf of $X$, so the space of point operators is

$$
\oplus_{p} \operatorname{Ext}^{p}\left(\mathcal{O}_{X}, \mathcal{O}_{X}\right)=\oplus_{p} H^{p}\left(\mathcal{O}_{X}\right)
$$

This agrees with [32]. Again, no quantum corrections are possible.

The algebra of local observables in any $N$-dimensional $\mathbb{Z}_{2}$-graded TFT is a supercommutative algebra with a Lie bracket of degree $N-$ $1 \bmod 2$. These two algebraic structures are compatible in an obvious sense. The Lie bracket arises as follows: to any local observable one can associate a tower of descendants. The $(N-1)^{\text {th }}$ descendant is a $N-1$ form of ghost number $1-N \bmod 2$ which is closed up to BRSTexact terms. Thus one can think of it as a conserved current which generates an infinitesimal symmetry of the TFT. All such symmetries form a Lie superalgebra with a bracket of degree 0 . One can show that the symmetries arising from descendants of local observables form a subalgebra of this Lie superalgebra, and therefore the algebra local observables inherits a Lie bracket of degree $N-1 \bmod 2$.

For example, in the curved B-model the algebra of local observables carries an odd bracket. A supercommutative algebra with a compatible 
odd Lie bracket bracket is called a $\mathbb{Z}_{2}$-graded Gerstenhaber algebra. It is well-known that the Hochschild cohomology of any $\mathbb{Z}_{2}$-graded DGcategory carries such a structure; in the case of the curved B-model the relevant category is the category of branes.

In the RW model, we expect that the algebra of local observables carries an even Lie bracket; the resulting structure is simply a Poisson algebra. Indeed, since $X$ is a complex symplectic manifold, the sheaf $\mathcal{O}_{X}$ is obviously a sheaf of Poisson algebras, and therefore its cohomology is a Poisson algebra. In the compact Kähler case the Poisson bracket is trivial; analogously, in the B-model with a compact Kähler target the Gerstenhaber bracket is trivial.

\section{Relationship With CATEGoRIFied ALGEBRAiC GEOMETRY}

In this paper we have studied topological boundary conditions and topological defects of various codimensions for the RW model. We have seen that boundary conditions form a 2-category: morphisms between two boundary conditions are boundary line operators separating two parts of the boundary, and 2-morphisms between line operators are local operator insertions on line operators. We have described a large class of boundary conditions for the RW model with target $X$, namely complex fibrations over complex Lagrangian submanifolds in $X$. The structure of this 2-category is rather intricate, and it would be desirable to have a more algebraic description for it. A good analogy is the Bmodel: the structure of the category of B-branes is greatly clarified by relating it to the category of coherent sheaves, i.e. a category of modules over the sheaf of holomorphic functions.

While we do not know such an algebraic description in general, we can propose a candidate description for the full sub-2-category whose objects are fibrations over a fixed complex Lagrangian submanifold $Y$. Let $\mathrm{A}_{0}$ denote the boundary condition corresponding to $\mathcal{Y}=Y$. Let A be any other boundary condition supported on $Y$. The category of boundary-changing line operators between $A_{0}$ and $A$ (i.e. the category $\operatorname{Hom}\left(\mathrm{A}_{0}, A\right)$ in the 2-category of boundary conditions) is a module over the monoidal category of boundary operators on $A_{0}$ (i.e. the category $\left.\operatorname{Hom}\left(\mathrm{A}_{0}, \mathrm{~A}_{0}\right)\right){ }^{8}$ If $\mathrm{A}$ is supported on $Y$, one might conjecture that the category $\operatorname{Hom}\left(\mathrm{A}_{0}, \mathrm{~A}\right)$ determines $A$; then one could hope to identify the 2 -category of boundary conditions with the 2-category of modules over the monoidal category $\operatorname{Hom}\left(A_{0}, A_{0}\right)$.

\footnotetext{
${ }^{8}$ Monoidal category is a categorification of an algebra. A module over a monoidal category is defined by categorifying the usual definition of a module over an algebra. Modules over a monoidal category form a 2-category.
} 
By analogy with the B-model, we expect that this conjecture is true if $Y$ is a (smooth) affine variety; otherwise we need to "sheafify" the categories $\operatorname{Hom}\left(A_{0}, A\right)$ and $\operatorname{Hom}\left(A_{0}, A_{0}\right)$, i.e. consider a sheaf of categories over $Y$ which is a module over a sheaf of monoidal categories.

On the classical level, we can make all this rather explicit. The monoidal category $\operatorname{Hom}\left(A_{0}, A_{0}\right)$ is the $\mathbb{Z}_{2}$-graded derived category of coherent sheaves on $Y$, or better the DG-category of $\mathbb{Z}_{2}$-graded complexes of $\mathcal{O}_{Y}$-modules, with the obvious monoidal structure. Any fibration $\mathcal{Y} \rightarrow Y$ with a curving $W$ is naturally a module over this category: the module structure is given by tensoring an object on $\mathcal{Y}$ by a pull-back of an object on $Y$.

Quantum corrections deform the monoidal category $\operatorname{Hom}\left(A_{0}, A_{0}\right)$, so for the boundary condition $A$ to make sense on the quantum level, the module $\operatorname{Hom}\left(\mathrm{A}_{0}, \mathrm{~A}\right)$ must admit a deformation as well. In general, as in the case of modules over an algebra, there are obstructions for finding such a deformation. This is what we found in section 2.5; the leading deformation turned out to be quadratic in the Atiyah class of the fibration $\mathcal{Y}$ and the curving $W$ on $\mathcal{Y}$ and linear in the class $[\beta]$. Presumably, all obstructions can be determined by studying the deformation theory of modules over the monoidal category $\operatorname{Hom}\left(\mathrm{A}_{0}, \mathrm{~A}_{0}\right)$.

As discussed above, deformations of $Y$ in $X$ are equivalent to turning on a nontrivial curving $W$ on $Y$. This suggests that the 2-category of modules over $\operatorname{Hom}\left(\mathrm{A}_{0}, \mathrm{~A}_{0}\right)$ also includes boundary conditions which are supported on a formal neighborhood $Y$. In general, this neighborhood is a deformations of $T_{Y}^{*}$, and the monoidal structure on the category $\operatorname{Hom}\left(\mathrm{A}_{0}, \mathrm{~A}_{0}\right)$ receives corrections from the classes $\beta^{(\ell)} \in H^{1}\left(\operatorname{Sym}^{\ell} T_{Y}\right)$ describing this deformation. Thus the RW model "solves" the problem of deformation quantization of the monoidal category $D_{\mathbb{Z}_{2}}(\operatorname{Coh}(Y))$.

A particularly simple and instructive case is when all classes $\beta^{(\ell)}$ vanish. In this case we can simply take $X=T_{Y}^{*}$. We conjecture that the 2-category of boundary conditions for the RW model with target $T_{Y}^{*}$ is equivalent to the 2-category of modules over the $\mathbb{Z}_{2}$-graded derived category of $Y$, or better the category of modules over the DG-category of complexes of $\mathcal{O}_{Y}$ modules, regarded as a monoidal DG-category. We can view an object of this 2-category as a sheaf of DG-categories over $Y$.

A very similar 2-category, called the 2-category of derived categorical sheaves over $Y$, was introduced in [36] (see also [4]). The aim of these papers was to develop a kind of categorification of algebraic geometry, with sheaves of DG-categories taking place of complexes of coherent sheaves. One apparent difference is that complex fibrations appear to 
be rather special sheaves of DG-categories, analogous in some sense to holomorphic vector bundles in the B-model. One may wonder if more general sheaves of categories, e.g. skyscraper sheaves, play a role in the RW theory. In analogy with the B-model, one could try to build more complicated boundary conditions by considering "complexes" of fibrations. However, it is not clear what this could mean. Instead, we will argue in [?] that skyscraper sheaves of DG-categories and other more exotic categorical sheaves appear in the RW theory automatically if we allow for nontrivial curving. In a sense, we will see that turning on a curving on a fibration is analogous to deforming a graded holomorphic vector bundle into a complex of holomorphic vector bundles.

Another apparent difference compared to $[36,4]$ is that the authors of $[36,4]$ work with $\mathbb{Z}$-graded complexes of $\mathcal{O}_{Y}$ modules, rather than with $\mathbb{Z}_{2}$-graded ones. We can account for this difference by noting that in the case $X=T_{Y}^{*}$ the $\mathbb{Z}_{2}$ ghost number symmetry of the RW model can be promoted to a $U(1)$ symmetry. Indeed, $T_{Y}^{*}$ has a $U(1)$ action with respect to which the holomorphic fiber coordinates have weight 2 . It preserves the metric and therefore the term $\mathcal{L}_{1}$ in the Lagrangian. On the other hand, the holomorphic symplectic form has weight 2 under this action, so this geometric $U(1)$ symmetry does not preserve $\mathcal{L}_{2}$. However, the term $\mathcal{L}_{2}$ is invariant under the combined action of the geometric $U(1)$ and the naive ghost number symmetry. We conclude that the RW model with target $T_{Y}^{*}$ has a $U(1)$ ghost number symmetry with respect to which the fiber coordinates on $T_{Y}^{*}$ have weight two. We can require all boundary conditions and line operators to be equivariant with respect to this symmetry.

The relationship between categorified algebraic geometry and $3 \mathrm{~d}$ TFT has been already pointed out in [4], from a somewhat different viewpoint. First of all, the definition of a $3 \mathrm{~d}$ TFT used in [4] is different from ours. Instead of considering the 3-category of decorated structures of dimension $n \leq 3$, the authors consider the $\infty$-category of two-dimensional cobordisms. The TFT is defined, roughly, as a functor from this $\infty$-category to the 2 -category of DG-categories. The authors of [4] construct a TFT in this sense for any complex manifold $Y$ (and more generally for any stack satisfying certain properties).

To compare with the discussion in [4], note that the TFT in the sense of [4] assigns a DG 2-category to a point, a braided monoidal DG-category to a circle and a DG-vector space to a Riemann surface. For a complex manifold $Y$, the 2-category assigned to a point is the 2category of categorical sheaves over $Y$. As discussed above, this agrees with the RW theory. (On the quantum level, one has to require $Y$ to be a Calabi-Yau manifold. This condition does not arise in [4], because 
the authors do not try to extend their theory to three-dimensional cobordisms.)

Further, according to [4] one assigns to a circle the DG-category of complexes of quasicoherent sheaves on the supermanifold $T_{Y}[-1]$ (i.e. the odd tangent bundle where the fiber coordinates have degree $-1)$. By Koszul duality, it is equivalent to the category of complexes of quasicoherent sheaves on the graded manifold $T_{Y}^{*}[2]$ (i.e. $T_{Y}^{*}$ with a $\mathbb{C}^{*}$-action such that the fiber coordinates have weight 2$)$. This is the same as the category of bulk line operators in the $\mathbb{Z}$-graded version of the RW model with target $T_{Y}^{*}$. In principle, one also needs to compare the braided monoidal structures on the two categories; we conjecture that they agree.

The unit object in the monoidal category attached to a circle is the structure sheaf of $T_{Y}^{*}$ (with the obvious $\mathbb{C}^{*}$-action). From the physical viewpoint, it represents the invisible line operator in the bulk. Its Dolbeault complex is the vector space attached to a 2 -sphere, or in physical terms the space of local operators in the bulk. The cohomology of this vector space can be identified with

$$
H^{\bullet}\left(\mathrm{Sym}^{\bullet} T_{Y}[2]\right)
$$

It is a supercommutative algebra with a compatible Poisson bracket of degree -2; as explained above, the Poisson bracket comes from the Poisson bracket on forms on $T_{Y}^{*}$. This graded Poisson algebra is the space of infinitesimal deformations of the RW model with target $T_{Y}^{*}$; from the mathematical viewpoint it describes infinitesimal deformations of the 2-category of modules over the monoidal DG-category of complexes of $\mathcal{O}_{Y}$ modules.

\section{Appendix A. The B-Model And its CATEgory of BRAnes}

A.1. BRST transformations, action, and observables. Let $X$ be a complex manifold with local complex coordinates $\phi^{i}, i=1, \ldots, n$. The fields of the B-model with target $X$ consist of a map $\phi: \Sigma \rightarrow X$, fermionic 0 -forms $\eta \in \Gamma\left(\phi^{*} \bar{T}_{X}\right)$ and $\theta \in \Gamma\left(\phi^{*} T_{X}^{*}\right)$, and a fermionic 1 -form $\rho \in \Gamma\left(\phi^{*} T_{X} \otimes T^{*} \Sigma\right)$. The BRST transformations are

$$
\begin{array}{rlrl}
\delta_{Q} \phi^{i} & =0, & \delta_{Q} \phi^{\bar{i}}=\eta^{\bar{i}}, \\
\delta_{Q} \eta^{\bar{i}}=0, & \delta_{Q} \theta_{i}=0 \\
\delta_{Q} \rho^{i}=d \phi^{i} . &
\end{array}
$$

The action is

$$
S_{0}=\int_{\Sigma}\left(\delta_{Q}\left(g_{i j} \rho^{i} \wedge * d \phi^{\bar{j}}\right)-\theta_{i} \nabla \rho^{i}-R_{j k l}^{i} \theta_{i} \rho^{j} \rho^{k} \eta^{\bar{l}}\right) .
$$


Here $g_{i j}$ is a Kähler metric on $X, \nabla$ is the covariant differential with respect to the pull-back of the Levi-Civita connection, and $R_{j k \bar{l}}^{i}$ is the curvature tensor of the Levi-Civita connection.

This action defines a topological field theory, because it depends on the worldsheet metric $h$ only through BRST-exact terms. It is called the B-model with target $X$ [42]. Its algebra of topological observables (i.e. the cohomology of $\delta_{Q}$ on the space of local functions of fields) is isomorphic to

$$
\mathcal{A}=\oplus_{p, q} H^{p}\left(\Lambda^{q} T_{X}\right) .
$$

The isomorphism acts as follows:

$$
\frac{1}{p ! q !} \omega_{\bar{i}_{1} \ldots \bar{i}_{p}}^{i_{1} \ldots i_{q}} d \phi^{\bar{i}_{1}} \ldots d \phi^{\bar{i}_{p}} \partial_{i_{1}} \ldots \partial_{i_{q}} \mapsto \frac{1}{p ! q !} \omega_{\bar{i}_{1} \ldots \bar{i}_{p}}^{i_{1} \ldots i_{q}} \eta^{\bar{i}_{1}} \ldots \eta^{\bar{i}_{p}} \theta_{i_{1}} \ldots \theta_{i_{q}}
$$

Correlators of topological observables depend only on the complex structure on $X$, because the variation of $S_{0}$ with respect to the metric $g_{i \bar{j}}$ is BRST-exact [42].

On the quantum level, one usually requires $X$ to be a Calabi-Yau manifold, i.e. one requires the existence of a holomorphic volume form $\Psi$ on $X$. The volume form is used to write down a BRST-invariant measure on the space of bosonic and fermionic zero modes. Given $\Psi$, one defines a trace function on $\mathcal{A}$ as follows: it is nonvanishing only on $H^{n}\left(\Lambda^{n} T_{X}\right)$ and is given by

$$
\omega \mapsto \int_{X} \Psi \wedge \omega\llcorner\Psi
$$

This trace function makes $\mathcal{A}$ into a Frobenius algebra.

The formula for the trace function makes sense in the case when the canonical line bundle $K_{X}$ of $X$ is not necessarily trivial, but its square is. We will call such $X$ a half-CY manifold. The closed sector of the B-model is well-defined in this slightly more general case.

The B-model has a $U(1)$ symmetry known as the ghost number symmetry. It acts trivially on the bosonic fields; the fermionic 0-forms have weight 1; the fermionic 1 -form $\rho$ has weight -1 . The BRST charge itself has weight 1 . One may choose to keep track only of the $\mathbb{Z}_{2}$ subgroup of the ghost number symmetry; the resulting theory is a $\mathbb{Z}_{2}$-graded version of the B-model.

A.2. Topological branes in the B-model. To any topological field theory one can associate a category of topological boundary conditions usually called the category of branes. In the case of the B-model with a Calabi-Yau target $X$, this is the bounded derived category of coherent sheaves (or more precisely, the bounded derived category of complexes of $\mathcal{O}_{X}$-modules with coherent cohomology). In the $\mathbb{Z}_{2}$ graded version of 
the theory, this presumably becomes the derived category of 2-periodic complexes of $\mathcal{O}_{X}$-modules with coherent cohomology.

Let us describe objects and morphisms in the category of B-branes. For definiteness, we will work with the $\mathbb{Z}_{2}$-graded version of the Bmodel. An object of the category of B-branes is a $\mathbb{Z}_{2}$-graded smooth vector bundle $E=E^{+} \oplus E^{-}$equipped with a flat $\bar{\partial}$-superconnection $D$. A flat $\bar{\partial}$-superconnection is an odd element of the $\mathbb{Z}_{2}$-graded vector space

satisfying

$$
\operatorname{End}_{\mathbb{C}}\left(\Omega^{0, \bullet}(E)\right)
$$

$$
\begin{aligned}
D^{2}=0, \quad D(\omega \wedge \sigma)=\bar{\partial} \omega \wedge \sigma+ & (-1)^{\operatorname{deg}_{\mathbb{Z}_{2}} \omega} \omega \wedge D \sigma, \\
& \forall \omega \in \Omega^{0, \bullet}(X), \forall \sigma \in \Omega^{0, \bullet}(E) .
\end{aligned}
$$

One can write $D$ as a sum of a $\bar{\partial}$-connection $\bar{\partial}_{E}: \Omega^{0, p}(E) \rightarrow \Omega^{0, p+1}(E)$ preserving the decomposition $E=E^{+} \oplus E^{-}$and an odd section of the $\mathbb{Z}_{2}$-graded vector bundle $\Omega^{0, \bullet}(\operatorname{End}(E))$. Note that $\bar{\partial}_{E}$ does not square to zero by itself, in general, so $E$ does not have a natural holomorphic structure. This description of B-branes goes back to Lazaroiu [27] and Diaconescu [12]; more recently it was discussed in [16, 5].

Given two such pairs $\left(E_{1}, D_{1}\right)$ and $\left(E_{2}, D_{2}\right)$ we consider the $\mathbb{Z}_{2^{-}}$ graded vector space

$$
\Omega^{0, \bullet}\left(\operatorname{Hom}\left(E_{1}, E_{2}\right)\right)
$$

and its odd endomorphism $D_{12}$ defined by

$$
D_{12} f=D_{2} f-(-1)^{\operatorname{deg}_{\mathbb{Z}_{2}} f} f D_{1}, \quad f \in \Omega^{0, \bullet}\left(\operatorname{Hom}\left(E_{1}, E_{2}\right)\right) .
$$

It is easy to see that $D_{12}^{2}=0$. We define the space of morphisms from $\left(E_{1}, D_{1}\right)$ to $\left(E_{2}, D_{2}\right)$ to be the cohomology of $D_{12}$.

Note that given a $\mathbb{Z}_{2}$-graded complex of holomorphic vector bundles $E$ on $X$ one gets an object of the category of B-branes by letting $D=\bar{\partial}+\mathcal{K}$, where $\mathcal{K}$ is the differential in the complex . The space of morphisms between two such objects is the hypercohomology vector spaces of the Hom-complexes. This shows that for smooth algebraic $X$ the derived category of 2-periodic complexes of coherent sheaves on $X$ is equivalent to a full subcategory of the category of B-branes. We conjecture that in general the $\mathbb{Z}_{2}$-graded version of the category of $\mathrm{B}$ branes is equivalent to the derived category of 2-periodic complexes of $\mathcal{O}_{X}$-modules with coherent cohomology. For a detailed discussion of the $\mathbb{Z}$-graded case see $[6,5,8]$.

To see how a pair $(E, D)$ defines a B-brane, we need to choose $\partial$ connections on $E^{+}$and $E^{-}$which in a local trivialization we write as

$$
\partial^{ \pm}=\partial+\mathcal{A}^{ \pm}, \quad \mathcal{A}^{ \pm} \in \Omega^{1,0}\left(U, \operatorname{End}\left(E^{ \pm}\right)\right), U \subset X .
$$


Further, we write

$$
D=\bar{\partial} \cdot \mathbb{1}+\mathcal{K}=\bar{\partial} \cdot \mathbb{1}+\left(\begin{array}{cc}
\mathcal{B}^{+} & \mathcal{T} \\
\mathcal{S} & \mathcal{B}^{-}
\end{array}\right)
$$

where

$$
\begin{aligned}
\mathcal{B}^{ \pm} \in \Omega^{0, \hat{1}}\left(U, \operatorname{End}\left(E^{ \pm}\right)\right), \mathcal{T} & \in \Omega^{0, \hat{0}}\left(U, \operatorname{Hom}\left(E^{-}, E^{+}\right)\right), \\
\mathcal{S} & \in \Omega^{0, \hat{0}}\left(U, \operatorname{Hom}\left(E^{+}, E^{-}\right)\right), U \subset X .
\end{aligned}
$$

Let us introduce the supermanifold $\Pi \bar{T}_{X}$ with odd coordinates $\eta^{\bar{i}}$. There is an obvious map $\pi: \Pi \bar{T}_{X} \rightarrow X$, and we may regard $\mathcal{K}$ as a locally-defined odd section of the $\mathbb{Z}_{2}$-graded bundle $\pi^{*} \operatorname{End}(E)$. Similarly, $\bar{\partial}$ can be reinterpreted as an odd vector field $\eta^{\bar{i}} \partial_{\bar{i}}$ on $\Pi \bar{T}_{X}$, and $D$ is a first-order differential operator on $\pi^{*} E$.

Now let $\gamma$ be a connected component of $\partial \Sigma$, i.e. a circle. Let $t \in[0,1)$ be a coordinate on $\gamma$. Given a map $\Phi=(\phi, \eta): \gamma \rightarrow \Pi \bar{T}_{X}$ and a section $\rho_{t}^{i} d t$ of $\phi^{*} T_{X} \otimes T_{\gamma}^{*}$, we consider a connection 1-form on the pull-back of $\Phi^{*}(E)$ :

$$
\mathcal{N}=\left(\begin{array}{cc}
\mathcal{A}_{i}^{+} d_{t} \phi^{i}+\mathcal{B}_{\bar{i}}^{+} d_{t} \phi^{\bar{i}}+F_{i \bar{j}}^{+} \rho_{t}^{i} \eta^{\bar{j}} & \rho_{t}^{i} \nabla_{i} \mathcal{T}+d_{t} \phi^{\bar{i}} \frac{\partial \mathcal{T}}{\partial \eta^{i}} \\
\rho_{t}^{i} \nabla_{i} \mathcal{S}+d_{t} \phi^{\bar{i}} \frac{\partial \mathcal{S}}{\partial \eta^{i}} & \mathcal{A}_{i}^{-} d_{t} \phi^{i}+\mathcal{B}_{\bar{i}}^{-} d_{t} \phi^{\bar{i}}+F_{i \bar{j}}^{-} \rho_{t}^{i} \eta^{\bar{j}}
\end{array}\right)
$$

Here $d_{t}=d / d t, \nabla_{i}$ denotes the covariant derivatives with respect to the $\partial$-connections on $\operatorname{Hom}\left(E^{ \pm}, E^{\mp}\right)$, and $F_{i \bar{j}}^{ \pm}$is defined by

$$
F_{i \bar{j}}^{ \pm} \eta^{\bar{j}}=\partial_{i} \mathcal{B}^{ \pm}-\partial_{j} \mathcal{A}_{i}^{ \pm} \eta^{\bar{j}}+\left[\mathcal{A}_{i}^{ \pm}, \mathcal{B}^{ \pm}\right]
$$

One can check that this connection on $\Phi^{*}(E)$ satisfies

$$
\delta_{Q}\left(d_{t}+\mathcal{N}\right)=\left[d_{t}+\mathcal{N}, \Phi^{*} \mathcal{K}\right]
$$

where the brackets denote the graded commutator. We can use this identity to construct a suitable boundary action as follows. Let $\mathcal{U}(0, t)$ be the parallel transport operator of the connection $\mathcal{N}$, i.e. the unique solution of the first order differential equation

$$
\left(d_{t}+\mathcal{N}\right) \mathcal{U}(0, t)=0
$$

satisfying $\mathcal{U}(0,0)=1$. We define

$$
\exp \left(-S^{b r y}\right)=\mathrm{S} \operatorname{Tr} \mathcal{U}(0,1)
$$

where STr denotes the supertrace on $\operatorname{End}_{\mathbb{C}}\left(E_{\Phi(0)}\right)$. Then it is easy to check that

$$
\delta_{Q} S^{b r y}=0 .
$$

Now suppose the circle $\gamma$ is split into two segments $\left(0, t_{0}\right)$ and $\left(t_{0}, 1\right)$ by the points $t=0$ and $t=t_{0}$. Suppose further than on the segment 
$\left(0, t_{0}\right)$ the boundary conditions is described by $\left(E_{1}, D_{1}\right)$, while on the segment $\left(t_{0}, 1\right)$ it is described by $\left(E_{2}, D_{2}\right)$. Given a morphism $f_{12}$ from $\left(E_{1}, D_{1}\right)$ to $\left(E_{2}, D_{2}\right)$ and a morphism $f_{21}$ from $\left(E_{2}, D_{2}\right)$ to $\left(E_{1}, D_{1}\right)$ consider the following expression to be inserted in the path-integral:

$$
\mathrm{S} \operatorname{Tr} f_{21}(\Phi(1)) \mathcal{U}_{2}\left(1, t_{0}\right) f_{12}\left(\Phi\left(t_{0}\right)\right) \mathcal{U}_{1}\left(0, t_{0}\right) .
$$

Here $\mathcal{U}_{1}$ and $\mathcal{U}_{2}$ are constructed from $\left(E_{1}, D_{1}\right)$ and $\left(E_{2}, D_{2}\right)$ as above, and we regard the bundle-valued forms $f_{12}$ and $f_{21}$ as sections of $\pi^{*} \operatorname{Hom}\left(E_{1}, E_{2}\right)$ and $\pi^{*} \operatorname{Hom}\left(E_{2}, E_{1}\right)$. It is easy to check that this expression is BRSTinvariant. Therefore we may regard morphisms $f_{12}$ and $f_{21}$ as local boundary topological observables to be inserted at the joining point of boundary conditions $\left(E_{1}, D_{1}\right)$ and $\left(E_{2}, D_{2}\right)$.

\section{Appendix B. The CuRved B-Model And its CATEgory of BRANES}

B.1. BRST transformations, action, and observables. The curved B-model can be thought of as a generalization of the Landau-Ginzburg deformation of the B-model, so we begin by briefly reviewing the latter.

Let $W$ be a holomorphic function on a manifold $X$ such that the line bundle $K_{X}^{2}$ is trivial. Then one can deform the B-model with target $X$ by modifying the transformation law for $\theta_{i}$ :

$$
\delta_{Q} \theta_{i}=-\partial_{i} W
$$

and adding to the action a term

$$
S_{W}=\frac{1}{2} \int \nabla_{i} \partial_{j} W \rho^{i} \rho^{j} .
$$

(Strictly speaking, to write down this deformation one needs only a closed holomorphic 1-form $d W$, not $W$ itself. However, if the cohomology class of $d W$ is nontrivial, the model does not admit interesting boundary conditions.)

We will call this model the Landau-Ginzburg model with target $X$ and superpotential $W$. Note that the postulated transformation law for $\theta$ and the action are compatible only with the $\mathbb{Z}_{2}$ subgroup of the ghost number symmetry. Thus the Landau-Ginzburg model is $\mathbb{Z}_{2}$-graded.

The usual action of the Landau-Ginzburg model differs from ours by a BRST-exact term

$$
-\int_{\Sigma} \operatorname{vol}_{\Sigma} \delta_{Q}\left(g^{i \bar{j}} \theta_{i} \partial_{\bar{j}} \bar{W}\right)=\int_{\Sigma} \operatorname{vol}_{\Sigma}\left(g^{i \bar{j}} \partial_{i} W \partial_{\bar{j}} \bar{W}-g^{i \bar{j}} \nabla_{\bar{k}} \partial_{\bar{j}} \bar{W} \theta_{i} \eta^{\bar{k}}\right),
$$

where $\mathrm{vol}_{\Sigma}$ is the volume form with respect to a Riemannian metric on $\Sigma$. 
The algebra of topological observables for the Landau-Ginzburg model is the hypercohomology of the complex

$$
\Lambda^{n} T_{X} \rightarrow \Lambda^{n-1} T_{X} \rightarrow \ldots \rightarrow T_{X} \rightarrow \mathcal{O}_{X}
$$

where the differential is contraction with the holomorphic 1-form $-\partial W$. In the case when $X$ is a contractible open subset of $\mathbb{C}^{n}$ and the critical points of $W$ are isolated, the hypercohomology of this complex is the Jacobi algebra

$$
H^{0}\left(X, \mathcal{O}_{X}\right) / A_{\partial W}
$$

where $A_{\partial W}$ is the ideal generated by partial derivatives of $W$. Note that this depends only on the behavior of $W$ in the neighborhood of the critical set of $W$. In particular, if $W$ has an isolated non-degenerate critical point, then the algebra is isomorphic to $\mathbb{C}$, and the LandauGinzburg model becomes trivial (such a model is called massive).

More generally, if the critical set of $W$ is a closed submanifold $T$ and $W$ is a Morse-Bott function (i.e. the Hessian of $W$ is non-degenerate in the directions normal to $T$ ), then the directions normal to $T$ are "massive" and can be integrated out. Thus the model becomes equivalent to the $\mathbb{Z}_{2}$-graded version of the B-model with target $T$. More precisely, this is true in the case when $\Sigma$ has empty boundary; if $\Sigma$ has boundaries and the number of transverse dimensions is odd, the Landau-Ginzburg model differs from the B-model with target $T$ in a subtle way (see below).

One can generalize the above construction by replacing $W$ with an inhomogeneous even $\bar{\partial}$-closed form on $X$. We will call this generalization the curved B-model. Consider a local observable $W(\phi, \eta)$ representing an even element of

$$
\oplus_{p} H^{p}\left(\mathcal{O}_{X}\right)
$$

Its descendants are

$$
\begin{aligned}
& W^{(1)}=\rho^{i} \partial_{i} W+d \phi^{\bar{i}} \frac{\partial W}{\partial \eta^{\bar{i}}} \\
& W^{(2)}=\frac{1}{2} \rho^{i} \rho^{j} \nabla_{i} \partial_{j} W+\rho^{i} d \phi^{\bar{j}} \partial_{i} \frac{\partial W}{\partial \eta^{\bar{j}}}+\frac{1}{2} d \phi^{\bar{i}} d \phi^{\bar{j}} \frac{\partial^{2} W}{\partial \eta^{\bar{i}} \partial \eta^{\bar{j}}}
\end{aligned}
$$

The descendants satisfy

$$
\delta_{Q} W^{(1)}=d W, \quad \delta_{Q} W^{(2)}=d W^{(1)}+\partial_{i} W \frac{\delta S_{0}}{\delta \theta_{i}} .
$$

We can deform the action of the B-model by a term

$$
S_{W}=\int_{\partial M} W^{(2)},
$$


and simultaneously modify the BRST transformation for $\theta_{i}$ :

$$
\delta_{Q} \theta_{i}=-\partial_{i} W
$$

Then the total action is BRST-invariant up to a total derivative:

$$
\delta_{Q}\left(S_{0}+S_{W}\right)=\int_{\Sigma} d W^{(1)}
$$

The resulting theory is the curved B-model. It is $\mathbb{Z}_{2}$-graded, just like the Landau-Ginzburg model.

The algebra of observables in the curved B-model is computed in essentially the same way as in the Landau-Ginzburg model. Namely, the algebra of observables is the cohomology of a certain differential $\delta_{Q}$ in the space of $(0, p)$ forms with values in polyvector fields of type $(q, 0)$. This differential is given by

$$
\delta_{Q}=\bar{\partial}-\partial W \mathbf{L}
$$

where $\llcorner$ denotes contraction on the holomorphic indices and exterior product on the antiholomorphic ones. If $X$ is compact and Kähler, then one can always find a form in the cohomology class of $W$ which is $\partial$-closed. For such $X$ the differential $\delta_{Q}$ reduces to $\bar{\partial}$, and the algebra of topological observables is the same as in the ordinary B-model.

B.2. Topological branes in the curved B-model. The category of branes associated to a curved B-model can be regarded as a deformation of the $\mathbb{Z}_{2}$-graded derived category; we will call it the curved derived category of $X$. There are several equivalent ways to define such a deformation; the most natural one from the physical viewpoint goes as follows. Let $W$ be an even $\bar{\partial}$-closed element of $\Omega^{0, \bullet}$. An object of the curved derived category is a $\mathbb{Z}_{2}$-graded smooth vector bundle $E=$ $E^{+} \oplus E^{-}$equipped with a curved differential $D$. A curved differential is an odd element of the $\mathbb{Z}_{2}$-graded vector space

$$
\operatorname{End}_{\mathbb{C}}\left(\Omega^{0, \bullet}(E)\right)
$$

satisfying

$$
\begin{aligned}
& D^{2}=\mathbb{1}_{E} \otimes W, \quad D(\omega \wedge \sigma)=\bar{\partial} \omega \wedge \sigma+(-1)^{\operatorname{deg}_{\mathbb{Z}_{2}} \omega} \omega \wedge D \sigma, \\
& \forall \omega \in \Omega^{0, \bullet}(X), \forall \sigma \in \Omega^{0, \bullet}(E) .
\end{aligned}
$$

One can write $D$ as a sum of a $\bar{\partial}$-connection $\bar{\partial}_{E}$ preserving the decomposition $E=E^{+} \oplus E^{-}$and an odd section of the $\mathbb{Z}_{2}$-graded vector bundle $\Omega^{0, \bullet}(\operatorname{End}(E))$. Note that $\bar{\partial}_{E}$ does not square to zero, in general, so $E$ does not have a natural holomorphic structure. 
Given two such pairs $\left(E_{1}, D_{1}\right)$ and $\left(E_{2}, D_{2}\right)$ we consider the $\mathbb{Z}_{2^{-}}$ graded vector space

$$
\Omega^{0, \bullet}\left(\operatorname{Hom}\left(E_{1}, E_{2}\right)\right)
$$

and its odd endomorphism $D_{12}$ defined by

$$
D_{12} f=D_{2} f-(-1)^{\operatorname{deg}_{\mathbb{Z}_{2}} f} f D_{1}, \quad f \in \Omega^{0, \bullet}\left(\operatorname{Hom}\left(E_{1}, E_{2}\right)\right) .
$$

It is easy to see that $D_{12}^{2}=0$. We define the space of morphisms from $\left(E_{1}, D_{1}\right)$ to $\left(E_{2}, D_{2}\right)$ to be the cohomology of $D_{12}$.

To see how an object of the curved derived category defines a brane for the curved B-model, we need to choose $\partial$-connections on $E^{+}$and $E^{-}$which in a local trivialization we write as

$$
\partial^{ \pm}=\partial+\mathcal{A}^{ \pm}, \quad \mathcal{A}^{ \pm} \in \Omega^{1,0}\left(U, \operatorname{End}\left(E^{ \pm}\right)\right), U \subset X .
$$

Further, we write

$$
D=\bar{\partial} \cdot \mathbb{1}+\mathcal{K}=\bar{\partial} \cdot \mathbb{1}+\left(\begin{array}{cc}
\mathcal{B}^{+} & \mathcal{T} \\
\mathcal{S} & \mathcal{B}^{-}
\end{array}\right)
$$

where

$$
\begin{aligned}
\mathcal{B}^{ \pm} \in \Omega^{0, \hat{1}}\left(U, \operatorname{End}\left(E^{ \pm}\right)\right), & \mathcal{T} \in \Omega^{0, \hat{0}}\left(U, \operatorname{Hom}\left(E^{-}, E^{+}\right)\right), \\
\mathcal{S} & \in \Omega^{0, \hat{0}}\left(U, \operatorname{Hom}\left(E^{+}, E^{-}\right)\right), U \subset X .
\end{aligned}
$$

Let us introduce the supermanifold $\Pi \bar{T}_{X}$ with odd coordinates $\eta^{\bar{i}}$. There is an obvious map $\pi: \Pi \bar{T}_{X} \rightarrow X$, and we may regard $\mathcal{K}$ as a locally-defined odd section of the $\mathbb{Z}_{2}$-graded bundle $\pi^{*} \operatorname{End}(E)$. Similarly, $\bar{\partial}$ can be reinterpreted as an odd vector field $\eta^{\bar{i}} \partial_{\bar{i}}$ on $\Pi \bar{T}_{X}$, and $D$ is a first-order differential operator on $\pi^{*} E$.

Now let $\gamma$ be a connected component of $\partial \Sigma$, i.e. a circle. Let $t \in[0,1)$ be a coordinate on $\gamma$. Given a map $\Phi=(\phi, \eta): \gamma \rightarrow \Pi \bar{T}_{X}$ and a section $\rho_{t}^{i} d t$ of $\phi^{*} T_{X} \otimes T_{\gamma}^{*}$, we consider a connection 1-form on the pull-back of $\Phi^{*}(E)$ :

$$
\mathcal{N}=\left(\begin{array}{cc}
\mathcal{A}_{i}^{+} d_{t} \phi^{i}+\mathcal{B}_{\bar{i}}^{+} d_{t} \phi^{\bar{i}}+F_{i \bar{j}}^{+} \rho_{t}^{i} \eta^{\bar{j}} & \rho_{t}^{i} \nabla_{i} \mathcal{T}+d_{t} \phi^{\bar{i}} \frac{\partial \mathcal{T}}{\partial \eta^{i}} \\
\rho_{t}^{i} \nabla_{i} \mathcal{S}+d_{t} \phi^{\bar{i}} \frac{\partial \mathcal{S}}{\partial \eta^{i}} & \mathcal{A}_{i}^{-} d_{t} \phi^{i}+\mathcal{B}_{\bar{i}}^{-} d_{t} \phi^{\bar{i}}+F_{i \bar{j}}^{-} \rho_{t}^{i} \eta^{\bar{j}}
\end{array}\right)
$$

Here $d_{t}=d / d t, \nabla_{i}$ denotes the covariant derivatives with respect to the $\partial$-connections on $\operatorname{Hom}\left(E^{ \pm}, E^{\mp}\right)$, and $F_{i \bar{j}}^{ \pm}$is defined by

$$
F_{i \bar{j}}^{ \pm} \eta^{\bar{j}}=\partial_{i} \mathcal{B}^{ \pm}-\partial_{\bar{j}} \mathcal{A}_{i}^{ \pm} \eta^{\bar{j}}+\left[\mathcal{A}_{i}^{ \pm}, \mathcal{B}^{ \pm}\right]
$$

One can check that this connection on $\Phi^{*}(E)$ satisfies

$$
\delta_{Q}\left(d_{t}+\mathcal{N}\right)=\left[d_{t}+\mathcal{N}, \Phi^{*} \mathcal{K}\right]-W^{(1)},
$$


where the brackets denote the graded commutator. We can use this identity to construct a suitable boundary action as follows. Let $\mathcal{U}(0, t)$ be the parallel transport operator of the connection $\mathcal{N}$, i.e. the unique solution of the first order differential equation

$$
\left(d_{t}+\mathcal{N}\right) \mathcal{U}(0, t)=0
$$

satisfying $\mathcal{U}(0,0)=1$. We define

$$
\exp \left(-S^{b r y}\right)=\mathrm{S} \operatorname{Tr} \mathcal{U}(0,1)
$$

where STr denotes the supertrace on $\operatorname{End}_{\mathbb{C}}\left(E_{\Phi(0)}\right)$. Then it is easy to check that

$$
\delta_{Q} S^{b r y}=-\int_{\gamma} W^{(1)}
$$

This precisely cancels the BRST-variation of the bulk action (121).

Now suppose the circle $\gamma$ is split into two segments $\left(0, t_{0}\right)$ and $\left(t_{0}, 1\right)$ by the points $t=0$ and $t=t_{0}$. Suppose further than on the segment $\left(0, t_{0}\right)$ the boundary conditions is described by $\left(E_{1}, D_{1}\right)$, while on the segment $\left(t_{0}, 1\right)$ it is described by $\left(E_{2}, D_{2}\right)$. Given a morphism $f_{12}$ from $\left(E_{1}, D_{1}\right)$ to $\left(E_{2}, D_{2}\right)$ and a morphism $f_{21}$ from $\left(E_{2}, D_{2}\right)$ to $\left(E_{1}, D_{1}\right)$ consider the following expression to be inserted in the path-integral:

$$
\mathrm{STr} f_{21}(\Phi(1)) \mathcal{U}_{2}\left(1, t_{0}\right) f_{12}\left(\Phi\left(t_{0}\right)\right) \mathcal{U}_{1}\left(0, t_{0}\right) .
$$

Here $\mathcal{U}_{1}$ and $\mathcal{U}_{2}$ are constructed from $\left(E_{1}, D_{1}\right)$ and $\left(E_{2}, D_{2}\right)$ as above, and we regard the bundle-valued forms $f_{12}$ and $f_{21}$ as sections of $\pi^{*} \operatorname{Hom}\left(E_{1}, E_{2}\right)$ and $\pi^{*} \operatorname{Hom}\left(E_{2}, E_{1}\right)$. It is easy to check that the BRST-variation of this expression cancels the BRST variation of $\exp \left(-S_{0}-S_{W}\right)$. Therefore we may regard morphisms $f_{12}$ and $f_{21}$ as local boundary topological observables to be inserted at the joining point of boundary conditions $\left(E_{1}, D_{1}\right)$ and $\left(E_{2}, D_{2}\right)$.

In the special case when $W$ is a holomorphic function on $X$, the curved B-model reduces to the Landau-Ginzburg model with superpotential $W$ and the curved derived category of $X$ reduces to the category of Landau-Ginzburg branes. In particular, if $X$ is a Stein manifold, the latter is equivalent to the category of matrix factorizations of $W$ $[19,9,28]$. An alternative algebro-geometric definition of this category was given by D. Orlov [29].

The category of Landau-Ginzburg branes has a remarkable property known as the Knörrer periodicity: its equivalence class does not change when one replaces $X$ with $X \times \mathbb{C}^{2}$ and $W$ with $W+x y$, where $x, y$ are coordinates on $\mathbb{C}^{2}[30]$. In particular, if $X=\mathbb{C}^{2 n}$ and $W$ is a nondegenerate quadratic form, Knörrer periodicity implies that the category of Landau-Ginzburg branes is equivalent $[19,30]$ to the category 
of $\mathbb{Z}_{2}$-graded vector spaces (which is the category of Landau-Ginzburg branes when $X$ is a point). On the other hand, if $X=\mathbb{C}^{2 n+1}$ and $W$ is a non-degenerate quadratic form, the category of Landau-Ginzburg branes is equivalent to the category of modules over the Clifford algebra with one generator [19]. It is easy to see that Knörrer periodicity remains true for the more general case of the curved derived category.

\section{Appendix C. Perturbative Computation of the fusion of WILSON LINES ON THE BOUNDARY}

In section 4.2 .3 we discussed that on the classical level fusion of the two line operators on the boundary corresponds to tensoring vector bundles and argued that the first quantum correction is the deformation of the resulting connection by a $(0,3)$-form

$$
C_{0} \beta_{\bar{m}}^{i j} F_{i \bar{k}} \tilde{F}_{j \bar{p}} d \phi^{\bar{m}} d \phi^{\bar{k}} d \phi^{\bar{p}}
$$

Here $C_{0}$ is a complex number, $F$ and $\tilde{F}$ are curvatures of connections on the two holomorphic vector bundles on $Y$, and $\beta_{\bar{m}}^{i j}$ is given by

$$
\beta_{\bar{m}}^{i j}=\Omega^{k^{\prime} i} \partial_{\bar{m}} A_{k^{\prime}}^{i}
$$

where $A_{k^{\prime}}^{K}$ is defined by $(22)$ and defines an embedding of $N_{Y}$ into $\left.T_{X}\right|_{Y}$. Essentially, $\beta_{\bar{m}}^{i j}$ is the second fundamental form of $Y$. In Darboux coordinates, $\beta_{\bar{m}}^{i j}$ is symmetric in the upper indices.

In this section we verify the presence of the correction (125) by computing the OPE of Wilson line operators on the boundary to leading order in $\beta$. To simplify the computation, note first that the BRSTinvariant connection 1-form entering the definition of the Wilson line

$$
\mathcal{O}^{(1)}(A)=A_{i} d \phi^{i}+A_{\bar{i}} d \phi^{\bar{i}}+F_{i \bar{j}} \chi^{i} \eta^{\bar{j}}
$$

is a descendant of a BRST-invariant 0-form observable

$$
\mathcal{O}(A)=A_{\bar{i}} \eta^{\bar{i}}
$$

That is, one has

$$
d \mathcal{O}(A)=\delta \mathcal{O}^{(1)}(A) .
$$

The observable $\mathcal{O}(A)$ does not determine $\mathcal{O}^{(1)}$ uniquely, but it does define it up to BRST-exact terms. Instead of computing the OPE of two Wilson lines, we may therefore compute the OPE of $\mathcal{O}(A)$ and $\mathcal{O}^{(1)}(\tilde{A})$, where $A$ and $\tilde{A}$ are connections on the two holomorphic vector bundles.

One can further simplify the computation by noting that the expected correction is antisymmetric with respect to the exchange of $A$ 
and $\tilde{A}$. The antisymmetric part of the quantum correction to the OPE is given by the contour integral over the insertion point of $\mathcal{O}^{(1)}(\tilde{A})$ :

$$
\oint_{\gamma} \mathcal{O}^{(1)}(\tilde{A}) \mathcal{O}(A)(0) .
$$

Here $\gamma$ is a circle at the boundary of $M$ centered at 0 . We are going to show that to leading order in $\beta$ this contour integral is

$$
C_{0} \beta_{\bar{m}}^{i j} F_{i \bar{k}} \tilde{F}_{j \bar{p}} \eta^{\bar{m}} \eta^{\bar{k}} \eta^{\bar{p}}
$$

The computation is performed by expanding around a constant bosonic background $\phi^{I}=\phi_{0}^{I}$, where $\phi_{0}^{I}$ belongs to $Y$. It is convenient to use a complex frame adapted to the boundary conditions. That is, we will work in the basis where the metric $g_{I \bar{J}}\left(\phi_{0}\right)$ does not have mixed components (with both primed and unprimed indices), while the holomorphic symplectic form $\Omega_{I J}\left(\phi_{0}\right)$ has only mixed components. In this coordinate system, $\beta_{\bar{m}}^{i j}$ is given by

$$
\beta_{\bar{m}}^{i j}=-\Omega^{k^{\prime} i} g^{\bar{k} j} \partial_{\bar{m}} g_{k^{\prime} \bar{k}}=\Omega^{i k^{\prime}} g^{i \bar{P}} g_{k^{\prime} \bar{J}} \Gamma_{\bar{m} \bar{P}}^{\bar{J}} .
$$

We will denote by $x$ a local complex coordinate on $\partial M$, while the coordinate in the normal direction will be denoted $x_{3}$. Thus we can expand the 1-form field $\chi^{I}$ as follows:

$$
\chi^{I}\left(x, x_{3}\right)=\rho^{I}\left(x, x_{3}\right) d x+\bar{\rho}^{I}\left(x, x_{3}\right) d \bar{x}+\chi_{3}^{I}\left(x, x_{3}\right) d x^{3} .
$$

We also use the notation that unprimed lower-case indices $i, j, k, \ldots$, run from 1 to $n$ and label coordinates on $Y$, while primed lower-case indices $i^{\prime}, j^{\prime}, k^{\prime}, \ldots$, run from $n+1$ to $2 n$ and label coordinates in the directions normal to $Y$.

The key ingredients in the computation are the following OPEs of local fields on $\partial M$ :

$$
\begin{aligned}
\rho^{i}(x, 0) \delta \phi^{j}(0,0) & \sim \frac{C}{x} \beta_{\bar{k}}^{i j} \eta^{\bar{k}}(0,0)+\ldots \\
\bar{\rho}^{i}(x, 0) \delta \phi^{j}(0,0) & \sim \frac{-C}{\bar{x}} \beta_{\bar{k}}^{i j} \eta^{\bar{k}}(0,0)+\ldots
\end{aligned}
$$

Here $\delta \phi^{i}=\phi^{i}-\phi_{0}^{i}, C$ is a complex number, and dots denote terms which are either nonsingular or contain derivatives of $\beta$.

Inspecting the action, we see that to leading order in perturbation theory we need to compute a free-field OPE

$$
\rho^{i}(x, 0) \delta \phi^{j}(0,0) \int d^{3} y \sqrt{h} g_{K} h^{\mu \nu} \chi_{\mu}^{K}\left(y, y_{3}\right) \partial_{\nu} \phi^{\bar{N}}\left(y, y_{3}\right) \Gamma_{\bar{N}}^{\bar{J}} \eta^{\bar{M}}\left(y, y_{3}\right) .
$$


The terms we are interested in are obtained by contracting $\rho^{i}$ with $\chi$ and $\delta \phi^{j}$ with $\phi^{\bar{N}}$. In the presence of the boundary the nonvanishing free-field contractions we need are given by

$$
\begin{aligned}
\delta \phi^{i}\left(x, x_{3}\right) \delta \phi^{\bar{j}}\left(y, y_{3}\right) & \sim \frac{g^{i \bar{j}}}{4 \pi|\mathbf{x}-\mathbf{y}|}+\frac{g^{i \bar{j}}}{4 \pi|\mathbf{x}-\Pi \mathbf{y}|^{2}} \\
\rho^{i}\left(x, x_{3}\right) \bar{\rho}^{k^{\prime}}\left(y, y_{3}\right) & \sim-\frac{i \Omega^{i k^{\prime}}}{8 \pi}\left(\frac{x_{3}-y_{3}}{|\mathbf{x}-\mathbf{y}|^{3}}-\frac{x_{3}+y_{3}}{|\mathbf{x}-\Pi \mathbf{y}|^{3}}\right), \\
\rho^{i}\left(x, x_{3}\right) \chi_{3}^{k^{\prime}}\left(y, y_{3}\right) & \sim \frac{i \Omega^{i k^{\prime}}}{8 \pi}\left(\frac{\bar{x}-\bar{y}}{|\mathbf{x}-\mathbf{y}|^{3}}+\frac{\bar{x}-\bar{y}}{|\mathbf{x}-\Pi \mathbf{y}|^{3}}\right), \\
\bar{\rho}^{i}\left(x, x_{3}\right) \chi_{3}^{k^{\prime}}\left(y, y_{3}\right) & \sim-\frac{i \Omega^{i k^{\prime}}}{8 \pi}\left(\frac{x-y}{|\mathbf{x}-\mathbf{y}|^{3}}+\frac{x-y}{|\mathbf{x}-\Pi \mathbf{y}|^{3}}\right), \\
\rho^{i}\left(x, x_{3}\right) \eta^{\bar{k}}\left(y, y_{3}\right) & \sim-\frac{g^{i \bar{k}}}{8 \pi}\left(\frac{\bar{x}-\bar{y}}{|\mathbf{x}-\mathbf{y}|^{3}}+\frac{\bar{x}-\bar{y}}{|\mathbf{x}-\Pi \mathbf{y}|^{3}}\right), \\
\bar{\rho}^{i}\left(x, x_{3}\right) \eta^{\bar{k}}\left(y, y_{3}\right) & \sim-\frac{g^{i \bar{k}}}{8 \pi}\left(\frac{x-y}{|\mathbf{x}-\mathbf{y}|^{3}}+\frac{x-y}{|\mathbf{x}-\Pi \mathbf{y}|^{3}}\right),
\end{aligned}
$$

where $\mathbf{x}, \mathbf{y} \in \mathbb{C} \times \mathbb{R}=\mathbb{R}^{3}$ have components $\left(x, x_{3}\right)$, (y, $\left.y_{3}\right)$, respectively, and $\Pi \mathbf{y} \in \mathbb{C} \times \mathbb{R}$ has components $\left(y,-y_{3}\right)$.

To detect the terms in the OPE we are interested in, it is convenient to integrate the expression (129) with respect to $x$ along a circle $\gamma_{\epsilon}$ of radius $\epsilon$ centered at $x=0$ and then take the limit $\epsilon \rightarrow 0$. The result is

$$
\beta_{\bar{k}}^{i j} \lim _{\epsilon \rightarrow 0} \int d^{3} y \eta^{\bar{k}}\left(y, y_{3}\right) F_{\epsilon}\left(y, y_{3}\right)
$$

where

$$
F_{\epsilon}\left(y, y_{3}\right)=\frac{-1}{16 \pi^{3}} \oint_{\gamma_{\epsilon}} d x \frac{\bar{x} y_{3}}{|\mathbf{y}|^{3}|\mathbf{x}-\mathbf{y}|^{3}}
$$

A straightforward computation which we omit shows that in the limit $\epsilon \rightarrow 0$ the function $F_{\epsilon}\left(y, y_{3}\right)$ tends to $C \delta^{3}\left(y, y_{3}\right)$ for some constant $C$ (in this computation we assume that $y^{3} \geq 0$, since the integration in (129) extends only over nonnegative $\left.y^{3}\right)$. This proves the first line of (127). The second line is proved similarly.

Now we can compute the contour integral (126). The result is (up to BRST trivial terms)

$$
-2 \pi i C F_{i \bar{j}} \tilde{F}_{k \bar{l}} \beta_{\bar{m}}^{i k} \eta^{\bar{j}} \eta^{\bar{l}} \eta^{\bar{m}} .
$$

This observable (divided by two) represents the leading quantum correction to the fusion of Wilson lines on the boundary. 


\section{REFERENCES}

[1] M. Atiyah, "Topological quantum field theories," IHES Publ. Math. 68, 175 (1988).

[2] J. C. Baez and J. Dolan, "Higher-dimensional algebra and topological quantum field theory," J. Math. Phys. 36, 6073 (1995).

[3] S. Bates and A. Weinstein, "Lectures on the geometry of quantization", Berkeley Mathematics Lectures Notes 8 (AMS, 1997).

[4] D. Ben-Zvi, J. Francis and D. Nadler, "Integral transforms and Drinfeld centers in derived algebraic geometry," arXiv:0805.0157.

[5] A. Bergman, "New Boundaries for the B-Model," arXiv:0808.0168 [hep-th].

[6] J. Block, "Duality and equivalence of module categories in noncommutative geometry I," arXiv:math/0509284.

[7] F. A. Bogomolov, "Hamiltonian Kählerian manifolds," Dokl. Akad. Nauk SSSR 243 (1978), p. 1101.

[8] A. Bondal and A. Rosly, to appear.

[9] I. Brunner, M. Herbst, W. Lerche and B. Scheuner, "Landau-Ginzburg realization of open string TFT," JHEP 0611, 043 (2006) [arXiv:hep-th/0305133].

[10] K. Costello, "Topological conformal field theories and Calabi-Yau categories," arXiv:math/0412149.

[11] L. Crane and D. Yetter, "On algebraic structures implicit in topological quantum field theories," J. Knot Theor. Ramifications 8, 125 (1999) [arXiv:hepth/9412025].

[12] D. E. Diaconescu, "Enhanced D-brane categories from string field theory," JHEP 0106, 016 (2001) [arXiv:hep-th/0104200].

[13] M. R. Douglas, "D-branes, categories and $\mathrm{N}=1$ supersymmetry," J. Math. Phys. 42, 2818 (2001) [arXiv:hep-th/0011017].

[14] D. S. Freed, "Higher algebraic structures and quantization," Comm. Math. Phys. 159, 343 (1994).

[15] V. W. Guillemin, "Clean intersection theory and Fourier integral operators," Lecture Notes in Math., vol. 459, Springer-Verlag, 1975, p. 23.

[16] M. Herbst, K. Hori and D. Page, "Phases Of N=2 Theories In 1+1 Dimensions With Boundary," arXiv:0803.2045 [hep-th].

[17] K. A. Intriligator and N. Seiberg, "Mirror symmetry in three dimensional gauge theories," Phys. Lett. B 387, 513 (1996) [arXiv:hep-th/9607207].

[18] M. Kapranov, "Rozansky-Witten invariants via Atiyah classes," Compositio Math. 115 (1999), 71.

[19] A. Kapustin and Y. Li, "D-branes in Landau-Ginzburg models and algebraic geometry," JHEP 0312, 005 (2003) [arXiv:hep-th/0210296].

[20] A. Kapustin and Y. Li, "Topological correlators in Landau-Ginzburg models with boundaries," Adv. Theor. Math. Phys. 7, 727 (2004) [arXiv:hepth/0305136].

[21] A. Kapustin and Y. Li, "Open string BRST cohomology for generalized complex branes," Adv. Theor. Math. Phys. 9, 559 (2005) [arXiv:hep-th/0501071].

[22] A. Kapustin and L. Rozansky, "On the relation between open and closed topological strings," Commun. Math. Phys. 252, 393 (2004) [arXiv:hepth/0405232].

[23] A. Kapustin and L. Rozansky, "Three-dimensional topological field theory and symplectic algebraic geometry II," in preparation. 
[24] A. Kapustin and E. Witten, "Electric-magnetic duality and the geometric Langlands program," arXiv:hep-th/0604151.

[25] K. Kodaira, "A theorem of completeness of characteristic systems for analytic families of compact submanifolds of complex manifolds," Ann. Math. (2) $\mathbf{7 5}$ (1962), 146.

[26] M. Kontsevich and Y. Soibelman, "Notes on $A_{\infty}$ algebras, $A_{\infty}$ categories and noncommutative geometry. I," arXiv:math/0606241.

[27] C. I. Lazaroiu, "Graded Lagrangians, exotic topological D-branes and enhanced triangulated categories," JHEP 0106, 064 (2001) [arXiv:hepth/0105063].

[28] C. I. Lazaroiu, "On the boundary coupling of topological Landau-Ginzburg models," JHEP 0505, 037 (2005) [arXiv:hep-th/0312286].

[29] D. Orlov, "Triangulated categories of singularities and D-branes in Landau-Ginzburg models," Tr. Mat. Inst. Steklova 246, 240 (2004) [arXiv:math/0302304].

[30] D. Orlov, "Triangulated categories of singularities and equivalences between Landau-Ginzburg models," arXiv:math/0503630.

[31] J. Roberts and S. Willerton, "On the Rozansky-Witten weight system," arXiv:math.DG/0602653.

[32] L. Rozansky and E. Witten, "Hyper-Kaehler geometry and invariants of threemanifolds," Selecta Math. 3, 401 (1997) [arXiv:hep-th/9612216].

[33] G. Thompson, "On the generalized Casson invariant," Adv. Theor. Math. Phys. 3, 249 (1999) [arXiv:hep-th/9811199].

[34] G. Tian, "Smoothness of the universal deformation space of compact CalabiYau manifolds and its Petersson-Weil metric," in Mathematical aspects of string theory, Proceedings of the conference held at the University of California, San Diego, California, 1986, ed. by S.-T. Yau, p. 629. World Scientific, Singapore, 1987.

[35] A. N. Todorov, "The Weil-Petersson geometry of the moduli space of $\mathrm{SU}(n \geq$ 3) (Calabi-Yau) manifolds. I.," Comm. Math. Phys. 126 (1989), 325.

[36] B. Toen and C. Vezzosi, "A note on Chern character, loop spaces and derived algebraic geometry," arXiv:0804.1274.

[37] C. Voisin, "Sur la stabilité des sous-variétés lagrangiennes des variétés symplectiques holomorphes," in Complex projective geometry (Trieste, 1989/Bergen, 1989), p. 294. Cambridge University Press, Cambridge, 1992.

[38] A. Weinstein, "On Maslov's quantization condition," Lecture Notes in Math., vol. 459, Springer-Verlag, 1975, p. 341.

[39] A. Weinstein, "Symplectic geometry," Bull. Amer. Math. Soc. 5, 1 (1981).

[40] E. Witten, "Topological Quantum Field Theory," Commun. Math. Phys. 117, 353 (1988).

[41] E. Witten, "Quantum Field Theory and the Jones Polynomial," Comm. Math. Phys. 121, 351 (1989).

[42] E. Witten, "Mirror manifolds and topological field theory," arXiv:hepth/9112056. 
California Institute of Technology

E-mail address: kapustin@theory.caltech.edu

University of North Carolina

E-mail address: rozansky@math.unc.edu

California Institute of Technology

E-mail address: saulina@theory.caltech.edu 